This working paper has not yet been peer-reviewed.

Latest update: $8 / 25 / 20$

\title{
Second-guessing of Spinoza: Psychophysiological and behavioral evidence that believing is default during proposition comprehension
}

\author{
Erik W. Asp ${ }^{1,2,3 *}$, Lila B. Khan ${ }^{2,3}$, Alec B. Jonason ${ }^{2,3}$, Melissa M. Adkins-Hempel ${ }^{4}$, Kelsey A. \\ Warner ${ }^{1,5}$, Enmanuelle Pardilla-Delgado ${ }^{6}$, Jessica D. Payne ${ }^{7}$, Paula Mullineaux ${ }^{2}$, Natalie L. \\ Denburg ${ }^{1}$, Daniel Tranel ${ }^{1,8}$ \\ ${ }^{1}$ Department of Neurology, University of Iowa, Iowa City, IA, USA \\ ${ }^{2}$ Department of Psychology, Hamline University, St. Paul, MN USA \\ ${ }^{3}$ Wesley \& Lorene Artz Cognitive Neuroscience Research Center, Hamline University, St. Paul, \\ MN, USA \\ ${ }^{4}$ Department of Clinical Pharmacology \& Toxicology, Hennepin Healthcare, Minneapolis, MN, \\ USA \\ ${ }^{5}$ Department of Speech-Language Pathology, Hennepin Healthcare, Minneapolis, MN, USA \\ ${ }^{6}$ Department of Psychiatry, Harvard School of Medicine, Massachusetts General Hospital, \\ Charlestown, MA, USA \\ ${ }^{7}$ Department of Psychology, University of Notre Dame, IN, USA \\ ${ }^{8}$ Department of Psychological and Brain Sciences, University of Iowa, Iowa City, IA, USA
}

Running title: Belief-default models, EDA, "aha”, and FFABB

The authors declare no competing interests.

*Correspondence:

Dr. Erik W. Asp

Department of Psychology

Hamline University

GLC $117 \mathrm{~W}$

1536 Hewitt Ave., St. Paul, MN 55114

easp02@hamline.edu

Keywords: belief, doubt, False Tagging Theory, belief-default model, Spinozan belief model, source memory, multinomial modeling, social cognition, credulity, familiarity, aha 


\begin{abstract}
The belief-default model contends that believing is inexorable during comprehension, and falsification is a subsequent, secondary process. By contrast, the Cartesian belief-fixation model argues that naïve propositions may be mentally represented without a truth or falsity stance. In the present research, data from four studies help adjudicate belief-fixation models, favoring the belief-default model: Studies 1-3 show that newly represented propositions are initially believed as the consequences of the truth from a naïve represented proposition will automatically activate contradictory mental information even when this processing impairs task performance (a "false" false alarm belief bias). Naïve propositions cannot be "merely" represented (without a truth stance) during comprehension. Studies 3 and 4 reveal unique electrodermal activity signals corresponding to propositions considered to be either true or false. We argue that the observed autonomic reactivity constitutes the source of two different epistemic emotions associated with the perceived outcomes of a memory search (i.e., "aha" and wrongness, respectively). To account for the psychophysiological results, we hypothesize that the epistemic emotion of familiarity is substantiated by an "aha" emotion which signals the recovery of represented propositions considered true during mnemonic processing. In addition, we show that anti-belief-default conclusions from recent investigations using multinomial processing tree modeling are tenuous as they depend on the type of false information paradigm employed. In sum, the data support the belief-default model and indicate a novel psychophysiological method to distinguish "believed" memory retrieval products from "guessed" responses derived via metacognitive strategies during veridical identification.
\end{abstract}




\section{Introduction}

"To conceive with passion is eo ipso to affirm."

$$
\text { William James (1905, p. 308) }
$$

"If you tried to doubt everything you would not get as far as doubting anything. The game of doubting itself presupposes certainty."

\section{Ludwig Wittgenstein (1969, OC §115)}

As our society has moved into the age of immediate digital social communication, it has become increasingly apparent that the growing abundance of misinformation is altering many individuals' behavior from the political domain to the financial (Lewandowsky et al., 2017; Vosoughi et al., 2018). Concern for the increased spread of misinformation has engendered a revitalization of research investigations that aim to understand the antecedents of credulity (Lazer et al., 2018; Lee \& Shin, 2019; Pennycook \& Rand, 2019). The sine qua non of understanding individual differences in credulity and skepticism are the psychological mechanisms of belief and doubt and their associated cognitive architecture (Porot \& Mandelbaum, 2020). The process that empowers cognitions into behavior (i.e., the mechanism that makes cognitions believed) and the process that denies a cognition its veracity resulting in behavioral inhibition (i.e., the mechanism that makes cognitions doubted), however, remain relatively obscure and under-researched. Several belief-fixation models have indicated that believing is default: it is inexorable with comprehension of some proposition. In this view, individuals are biased toward belief simply due to the ease of belief and relative difficulty of disbelief. Understanding the spread of misinformation may be aided by validation of belief- 
default models as mass false idea exposure would result in substantial acceptance by those who do not have the cognitive resources, correct information, motivation, or neural integrity to perform the mental work to doubt the idea.

\section{Psychological Models of Belief and Doubt}

Investigations into belief and doubt mechanisms have largely focused on two contrasting models named after the $17^{\text {th }}$ century philosophers who inspired them: René Descartes and Baruch Spinoza (Gilbert, 1991) ${ }^{1}$. The process of belief fixation involves two stages: 1) comprehension of some proposition, and 2) assessment of that proposition with other extant mental information. The product of propositional assessment is a labeling or tagging of the assessed truth value to that proposition which could be indexed in the future (Gilbert, 1991). The Cartesian belief model argues that belief fixation of a proposition occurs via two separate and sequential stages (Figure 1). The understanding of a proposition precedes and is independent from the assessment and labeling of the cognition as true or false. Thus, in the Cartesian perspective propositions that have been represented must be empowered by a truth tag to induce cognition-consistent action. At any given time, then, three states are possible for any given represented proposition (Figure 1): 1) a proposition without a true or false tag (which is neither believed nor disbelieved), 2) a proposition with a true tag (which is believed), and 3) a proposition with a false tag (which is disbelieved). The Spinozan belief model argues that the process of comprehension itself produces a believed represented proposition (Figure 1). Thus, cognitions are empowered in the Spinozan belief model by mere representation (Gilbert, 1991). They are ballistic; they will induce cognition-consistent action, per se (Mandelbaum, 2014). It is important to note that

\footnotetext{
${ }^{1}$ There is some doubt whether Descartes and Spinoza would agree with all aspects and implications of their respective models (for a discussion, see Mandelbaum, 2014). In this vein, several scholars have suggested the better term may be "Spinozistic" rather than "Spinozan" belief model; however, for the sake of continuity we will use the conventional nomenclature.
} 
assessment can follow proposition representation in the Spinozan belief model. A subsequent, secondary psychological process can produce disbelief by the addition of a false tag, but no process uniquely labels propositions with true tags during initial comprehension and assessment in the Spinozan belief model. Here, two states are possible for any given represented proposition (Figure 1): 1) a proposition without a false tag (which is believed), and 2) a proposition with a false tag (which is disbelieved).

Thus, the Spinozan belief model may be termed a belief "default" model in the sense that, if a proposition is mentally represented, it is believed and will alter the behavioral tendencies of the receiver ${ }^{2}$ until, at a later time, it is assessed and assigned a false tag. Represented propositions (without an associated false tag) do not need to be turned into belief, they are, in fact, beliefs. The False Tagging Theory (FTT) is a subsequent belief-default model which elaborates the Spinozan belief perspective in three critical domains (Asp \& Tranel, 2013). First, the FTT argues for unique neuroanatomical region functionality for the processes of belief and doubt. Second, the FTT delineates a continuous (rather than discrete) tagging process, where more false tags (or one false tag which may vary in strength) may result in less belief and increased doubt toward a particular proposition ${ }^{3}$ (Figure 1). This model suggests that false tagging is an analogue signal (as opposed to digital) producing many possible states for any given represented proposition with varying degree of belief outcomes as a result (Figure 1). Finally, the FTT specifies that veridical identification is an affective process mediated by

\footnotetext{
2 This assumes the proposition in question is activated during the period necessary for belief-consistent behavior (Bendana \& Mandelbaum, in press).

${ }^{3}$ In a personal communication, Dr. Daniel Gilbert detailed that he never conceived of tags as discrete events without the possibility of varying degrees of belief as outcomes of an analogue-type signal. Gilbert's (1991) metaphors used to clarify the belief models (i.e., library tagging systems) may have been responsible for later researchers adapting discrete tags in their framework building evidence for the two models. Nonetheless, a continuous false tagging signal may be in accordance with the original author's intent of the Spinozan belief model.
} 
somatic, bioregulatory tags. Thus, the FTT predicts that veridical identification should be distinct from general source memory identification using indirect measurements of sympathetic nervous activity with electrodermal activity (EDA) as an index (Dawson et al., 2000).

It is also important to note that belief-default models do not preclude a secondary process increasing confidence or certainty toward mentally represented propositions. The fundamental attribute of belief-default models is whether or not naïve propositions are initially represented with a "truth stance" (Figure 1). That is, does the process of comprehension itself activate extant mental information consistent with the truth of the naïve proposition (i.e., belief-default models) or following comprehension, assessment, and affixation of a "true tag" is extant mental information consistent with the truth of the naïve propositions elicited (i.e., Cartesian belief model)? If no relevant extant contradictory mental information is timely activated or is not present in an individual's store of mental information, then belief-default models predict the naïve proposition will induce cognition-consistent behavior (given appropriate circumstances and activation of the relevant mentally-represented proposition) $)^{4}$. However, the Cartesian belief model predicts that a naïve proposition not affixed with a true or false tag will not induce cognition-consistent behavior in the same scenario as above. Thus, the validity of belief-default models does not rest on whether "true tags" or mechanisms to increase confidence/certainty in propositions and the likelihood of cognition-consistent behavior exist, but rather on how propositions proper (i.e., without true or false tags) are represented and effectuate cognition/behavior (i.e., cognitive architecture, Figure 1).

Epistemic emotions and autonomic arousal

\footnotetext{
${ }^{4}$ See Spinoza's (1982) winged horse example in the $49^{\text {th }}$ proposition of Part 2 of The Ethics.
} 
A central perspective on the operations of emotions and feelings holds that afferent visceral signals form a core element of subjective feelings which influence cognitive states and choices in decision-making scenarios (Damasio, 1994; Damasio \& Carvalho, 2013). Both covert and overt emotional states (the latter which may be experienced as conscious feelings) are substantiated from changes in the representations of bodily states or 'action programmes'. Beyond the traditional, basic emotions (e.g., disgust, fear, anger) are emotions in various cognitive domains that refer to unique phenomenological experiences associated with memory or knowledge (Arango-Munoz, 2014). "Epistemic" feelings include such experiences as familiarity, déjà vu, uncertainty, confidence, forgetting, knowing, "aha”, or wrongness. Recent research has shown that such epistemic experiences are directly related to 'action programmes' and visceral autonomic feedback which can be indexed via EDA (Fiacconi et al., 2017; Morris et al., 2008; Shen et al., 2018). Generally, this perspective suggests emotions (including epistemic emotions) are not a byproduct of cognitive functioning, but play an integral role influencing cognition and subsequent behavior (Bechara et al., 1997; Damasio, 1994; Schwarz, 2012). The FTT hypothesizes veridical identification during a true/false recognition memory task will produce distinct EDA signals reflective of epistemic emotions. Illusory truth, familiarity, and "aha”

During recognition and recall memory tasks, identification of familiar, previously seen items correspond to an increase in autonomic arousal that may be indexed via EDA (e.g., Morris et al., 2008; Plouffe \& Stelmack, 1984; Shearer \& Mikulka, 1996; Stelmack et al., 1983; Tranel et al., 1985; Volz et al., 2017). The epistemic feeling of familiarity has been associated with positive affect (e.g., Duke et al., 2014; Garcia-Marques et al., 2004), and robustly biases truth judgments (i.e., the illusory truth effect, Begg et al., 1992; Dechene et al., 2010; Hasher et al., 
1977). Indeed, repetition increases truth judgments even for conspicuous, known false propositions (Fazio, 2020; Fazio et al., 2015). However, the familiarity-truth link has long puzzled researchers as "there is no logical reason for repetition to affect rated truth or for earlier information to be trusted more than later information” (Begg et al., 1992, p. 447). Explanations tend to appeal to an ecological validity assumption (e.g., Unkelbach \& Stahl, 2009): associations between familiarity and truth are learned over time as most information we are exposed to is true (Grice, 1975) and true information is often repeated. However, unlike other fluency-truth associations (Unkelbach, 2007), repetition effects are not easily reversed (Garcia-Marques et al., 2016; Silva et al., 2016). Thus, simple learned associations between repeated statements and truth verification appear inadequate as a complete explanation for the illusory truth effect.

Instead, we suggest the epistemic feeling of familiarity is substantiated by another epistemic emotion with more direct connections to truth: the "aha" experience (Laukkonen, Webb, et al., 2018). Like familiarity, "aha" is a positive valanced emotion that is associated with certainty and easiness (Shen et al., 2018). Self-reported "aha" experiences robustly predict correct answers (Salvi et al., 2016; Webb et al., 2018) with large effect sizes to problem accuracy $(d=1.64)$ and self-rated confidence ( $d=1.87$, Laukkonen, Ingledew, et al., 2018). Importantly, "aha" also biases truth judgments to both true and false propositions when artificially-induced (Laukkonen et al., 2020).

Here, we propose that covert "aha" emotions signal the recovery of information considered true following a memory search ${ }^{5}$. Thus, increased EDA for familiar stimuli (Morris

\footnotetext{
${ }^{5}$ Successful memory searches would not be viewed as traditional insight problems that resolve an impasse with an "aha" experience; however, positive emotions, feelings of easiness, and feelings of certainty are shared between feelings of "aha" (Shen et al., 2018) and familiarity (for a discussion regarding the mechanisms of "aha" and insight, see Weisberg, 2015). Moreover, self-reported "aha" is not exclusive to traditional insight problems (Webb et al., 2018). Indeed, the correct solutions to anagrams (that most often elicit conscious "aha" relative other traditional insight problems, Webb et al., 2018) are dependent on successful memory searches of the mental lexicon.
} 
et al., 2008) represent covert "aha" emotions (Bechara \& Damasio, 2005; Shen et al., 2018) resulting from a successful ${ }^{6}$ memory search. If belief-default models are correct, and propositions considered true are mentally represented without true or false tags (Figure 1), then correct identification of previously seen items (considered true) should also result in an increased EDA signal (i.e., an "aha" emotion) relative to correct foil identification (i.e., new items not previously seen, and not eliciting an "aha" emotion). In this perspective, "aha" is specific to products of memory searches as it signals the recovery of information from memory considered true that matches a given stimulus. Thus, in both yes/no and true/false recognition memory tasks (e.g., Gilbert et al., 1993), propositions represented without tags that are retrieved from memory successfully will correspond to increased EDA.

Critically, this proposed "aha" mechanism indexing true propositions during memory retrieval is distinct from the theoretical "true tags" of the Cartesian belief model. Because all naïve propositions need to be empowered by a "truth tag" in the Cartesian belief model, "aha" experiences would be necessary for every naïve proposition to create cognition-consistent behavior. Indeed, it is a challenge to the Cartesian belief model proponent what process could assign truth to the constant, voluminous incoming data from cognitive and perceptual systems to ensure timely, accurate, and adaptive behavior (Pennycook et al., 2019). Rather, we restrict our proposed "aha" mechanism for truth judgments exclusively to mnemonic processing. Only following a perceived successful memory search will covert "aha" emotions and increased EDA be elicited.

Remembered false information, wrongness, and the FTT

\footnotetext{
${ }^{6}$ More specifically, a covert "aha" emotion may correspond to a "perceived" successful memory search as EDA does not distinguish between true memories and "believed" false memories (see Volz et al., 2017).
} 
However, the FTT predicts the elicitation of a different covert epistemic emotion following the recovery of information from memory considered false. The correct identification of previously seen items labeled as false should result in a reactivation of a remembered false tag accompanied by a change in the bodily state (Asp \& Tranel, 2013) that may be indexed via EDA measurements. Thus, we predict correct identification of previously seen items labeled as false will produce 1) an increased EDA signal relative to correct foil identification and 2) a difference in EDA magnitude relative to correct identification of previously seen items labeled as true (i.e., a covert "aha" emotion). The FTT postulates that this EDA signal is reflective of a negative epistemic emotion (i.e., when expressed consciously, the phenomenological experience of dissonance or wrongness) that is distinct from the positive "aha" experience (Asp \& Tranel, 2013; Elkin \& Leippe, 1986; Gawronski \& Strack, 2004; Harmon-Jones, 2000; Shen et al., 2018). These affective states are theorized have a direct role influencing truth judgments as the induction of positive emotional states promotes credulity and the induction negative emotional states promotes skepticism (Forgas, 2019; Koch \& Forgas, 2012). A central aim of the present investigations was to examine these autonomic feedback-related hypotheses and the resulting implications for belief-fixation models (Studies 3 and 4).

\section{Evidence for Belief-Default Models}

Early empirical results contrasting the Cartesian and Spinozan belief models favored the belief-default perspective. Gilbert and colleagues (Gilbert et al., 1990; Gilbert et al., 1993) conducted six separate studies demonstrating that cognitive load during the presentation of explicitly-labeled false information acts to increase the likelihood that participants will misremember the false information as true. For instance, during the Hopi Language Task (HLT, Study 1, Gilbert et al., 1990) participants needed to learn an ostensibly foreign language. They 
were presented propositions in the form An $X$ is a $Y$, where $X$ was a Hopi Indian noun and $Y$ was its English equivalent. Following each proposition, another screen indicated that the proposition was either true or false. For some of the trials a distracter task was given following the veridical signal. A subsequent true/false recognition memory test showed that the distracter task specifically decreased correctly identifying false Hopi propositions as false (FasF responses) and increased incorrectly identifying false Hopi propositions as true (FasT errors, see Table 1 for a listing of possible response outcomes during traditional true/false recognition memory tests). Critically, the distracter task did not affect correctly identifying true Hopi proposition as true (TasT responses) nor incorrectly identifying true Hopi propositions as false (TasF errors). Gilbert et al. (1990) argued that the cognitive load interrupted the belief assessment process which tended to leave propositions in the represented and believed state of the Spinozan model instead of the represented but neither believed nor disbelieved state of the Cartesian model (Figure 1). Gilbert and colleagues replicated these basic findings using propositions labeled true or false embedded in a narrative structure (Experiment 1, Gilbert et al., 1993). Moreover, when the explicitly-labeled false propositions exacerbated a protagonist's crime, cognitive load acted to increase prison sentencing of the protagonist. Similarly, when the explicitly-labeled false propositions extenuated a protagonist's crime, cognitive load acted to decrease prison sentencing of the protagonist. Thus, distracted participants behaved in accordance with credulity to the false propositions and uniquely misremembered the false propositions as true (FasT errors) during a recognition memory test.

These narrative-based false information paradigm results were replicated and extended in three subsequent developmental studies comparing younger and older adults (Chen, 2002; Chen \& Blanchard-Fields, 2000). Several researchers have noted that younger adults under conditions 
of divided attention tend to mimic older adults' task performance (e.g., Craik, 1982). In accordance, Chen and colleagues (Chen, 2002; Chen \& Blanchard-Fields, 2000) found that older adults under full attention (without cognitive load) were as swayed by explicitly-labeled false propositions during prison sentencing (and other social judgments of protagonists) as younger adults under cognitive load. Older adult recognition memory test performance was also equivalent with younger adult (under cognitive load) performance. That is, older adults under full attention had increased FasT errors relative to younger adults under full attention (Table 2). Impairments in falsification processes have been attributed to less efficient strategic memory mechanisms (e.g., Prull et al., 2006) and impairments in prefrontal cortex functioning (Asp et al., 2012; Asp \& Tranel, 2013; Denburg et al., 2007). Older adults' specific deficit in remembering that a proposition was previously labeled as false or was incorrect may account for their increased vulnerability to the illusory truth effect (e.g., Dechene et al., 2010; Skurnik et al., 2005) and the continued influence effect of misinformation (Swire et al., 2017). Thus, FasT errors on true/false recognition memory tests may offer a sensitive measure indexing individual differences in credulity.

\section{Evidence against Belief-Default Models}

In the last 15 years there has been a steadily-growing series of empirical studies with purported evidence against the Spinozan/belief-default models (e.g., Hasson et al., 2005; Nadarevic \& Erdfelder, 2013, 2019; Richter et al., 2009; Street \& Kingstone, 2017; Street \& Richardson, 2015). These anti-belief-default model arguments largely fall into two categories: Doxastic processing context-dependence and increased truth guessing bias effects. Contextdependence arguments (e.g., Hasson et al., 2005; Richter et al., 2009) claim that the style of doxastic processing (whether it is a Cartesian or a belief-default procedure) may be altered by the 
content of the proposition to which one is exposed. However, as Mandelbaum (2014) has cogently argued, the inability to gain propositional information prior to propositional understanding precludes this possibility.

A thornier challenge to belief-default models has been raised by arguments suggesting that cognitive load-induced increased FasT errors during false information paradigms do not represent "believed" memory errors but in fact reflect an uncertainty state followed by an increased "guessing" true bias (Nadarevic \& Erdfelder, 2013, 2019; Street \& Kingstone, 2017; Street \& Richardson, 2015). Street and Kingstone (2017) argued that if individuals are unsure regarding the veridical status of the memory item and forced to guess, they will use the general heuristic that most information communicated tends to be true (Grice, 1975) and thus guess true. Street and Kingstone (2017) found that distraction failed to increase the FasT error ${ }^{7}$ rate when an uncertainty option was introduced. Thus, when participants were given the possibility to select a response that reflects an uncertain memory state during the recognition memory test the critical error rate increase supporting belief-default models was not observed. Gilbert and colleagues (1990) anticipated this objection and marshaled three main arguments against the increased true guessing bias criticism: 1) There was no evidence of a general guessing true bias when items could not have been in memory. That is, when participants misidentified unseen foil items (i.e., never seen items, NS), they tended to answer false rather than true (more foil as false (NSasF) errors, than foil as true (NSasT) errors, see Table 1). 2) There was no evidence of increased guessing processing using the reaction time data: FasT errors following the distraction manipulation were not longer than FasT errors without distraction. 3) Finally, during the

\footnotetext{
${ }^{7}$ Options for the participants in the Street and Kingstone (2017) study were Truth, Lie, and Unsure as this was a deception detection experiment. Similar experimental designs have been used in support of the Spinozan belief model (see Study 2, Gilbert et al., 1990).
} 
narrative-based false information paradigm, participants behaved in accordance with the explicitly-labeled false statements prior to the production of increased FasT errors (Gilbert et al., 1993). Thus, it is difficult to understand how information that presumably existed in a state of uncertainty (neither true nor false) swayed the behavior of the participants before the recognition memory test. This circumstantial evidence notwithstanding, there remain doubts regarding whether the critical FasT errors are actually "believed" or merely "guessed" based on a Gricean heuristic (Nadarevic \& Erdfelder, 2013). While belief-default models do not specify that all FasT errors are believed (i.e., they allow that guessing based on a Gricean heuristic may occur), the majority of FasT errors should be "believed". Thus, "believed" FasT errors should be greater than "guessed" FasT errors and "believed" TasF errors on standard true/false recognition memory tests. If the FTT's prediction of veridical identification during a true/false recognition memory test as affective processes (which could be indexed via EDA) is correct, then EDA data may directly identify FasT errors as "believed" or merely "guessed." This is a secondary aim the current studies sought to investigate.

Another fashionable technique for distinguishing between "believed" memory outcomes and guessing rates is with the use of multinomial processing tree (MPT) models, which purportedly may be used to disentangle memory processes from guessing processes given a set of assumptions regarding the different underlying processes (Batchelder \& Riefer, 1990; Erdfelder et al., 2009; Riefer \& Batchelder, 1988). Nadarevic and Erdfelder (2013) have used a two-high-threshold (2HT), three-source variant MPT model (Meiser \& Broder, 2002; Riefer et al., 1994), common in source memory research (Erdfelder et al., 2009), to argue that the critical increased FasT errors for belief-default models result from an increased tendency for individuals to guess true rather than false when context memory declines (see also Nadarevic \& Erdfelder, 
2019). This conclusion is based on a high true guessing parameter ( $\left.a_{\text {Hans/true }}\right)$ relative to a lower false guessing parameter ( $\left.a_{\text {Paulffalse }}\right)$ during the 1-week condition (Experiment 2, Nadarevic \& Erdfelder, 2013). Unfortunately, Nadarevic and Erdfelders' (2013) conclusions rely on four unfounded assumptions regarding belief-default models and their MPT data.

Assumption 1: Veridical identification (i.e., remembering an item as true or false) uses the same mental processes as source memory identification (e.g., remembering if an item was associated with attribute A or B). Two-high-threshold veridical MPT models assume discrete and sequential cognitive states (such as item recognition and source discrimination; e.g., Bayen et al., 2000) to estimate parameter probabilities (Nadarevic \& Erdfelder, 2013). Specifically, for cognitive states resulting from the true source, standard 2HT MPT models are inadequate to examine belief-default model cognitive architecture. In the belief-default perspective there are no true tags (Figure 1). Thus, all items recognized that do not have associated false tags are regarded as true to the individual. A MPT model that would reflect belief-default model cognitive architecture should not include a parameter designed to measure the specific probability of detecting the correct source of true statements (e.g., parameter $d_{\text {Hans/true }}$, Nadarevic \& Erdfelder, 2013). However, belief-default model cognitive architecture does require two separate and sequential cognitive states for correctly identifying false information. Thus, correct FasF responding requires correct item recognition followed by correct false tag identification. If an item from the false source is correctly recognized but there is not correct false tag identification, then the item from the false source is considered true, producing a FasT error. Reaction time data on true/false verification tasks supports the belief-default model as correctly identifying false information takes more time than correctly identifying true information (Carpenter \& Just, 1975; Gilbert et al., 1990; Gough, 1965; Harris et al., 2008). The belief- 
default perspective suggests that this additional step of false tag identification is responsible for the time lag. Moreover, Nadarevic and Erdfelders' (2013) data indicate a clear performance advantage during veridical identification as opposed to source memory (i.e., only the names of the trivia sources) identification (precue versus postcue condition comparison, Experiment 1, Nadarevic \& Erdfelder, 2013) which suggests the two processes are distinct (see also Begg et al., 1992). Therefore, the cognitive architecture and the sequential mental processes implied by belief-default models preclude the valid use of standard source memory 2HT MPT models.

Assumption 2: Propositions that are tagged will produce superior memory performance relative to untagged propositions, granting evidence toward the belief models. Differential memory performance solely based on which propositions are associated with tags is an assertion not consistent with belief-default models. Nadarevic and Erdfleder (2013, p. 179) state, "if people store 'false' tags only, as predicted by the Spinozan model, good source memory in the precue condition should be limited to the false statements of Paul." Because there is an additional step of correct false tag identification following item recognition (relative to true identification), belief-default models actually predict the opposite conclusion: even without distraction/cognitive load there should be a slight advantage for correctly remembering true information (TasT responses) compared to correctly remembering false information (FasF, see also Pantazi et al., 2018).

Assumption 3: An uncertain cognitive state regarding propositions in belief-default models is possible without associated false tags. In the belief-default perspective, any proposition that does not have an associated false tag is considered true and is believed (Figure 1). In the FTT, uncertainty regarding a proposition is produced by a minimal number of false tags (or one "weak" false tag); whereas disbelief regarding a proposition is produced by a 
maximal number of false tags (or one "strong" false tag). Thus, in this continuous tagging perspective, correct responses in uncertain categories may be correct (weak) FasF responses (e.g., Fritz/random, Nadarevic \& Erdfelder, 2013) ${ }^{8}$.

Assumption 4: Biased guessing strategies using base-rate information do not influence the MPT results and belief model conclusions. Parameters estimated in MPT models may be systematically biased by various metacognitive guessing influences (Batchelder \& Batchelder, 2008; Kuhlman \& Bayen, 2016). During recognition memory tests, participants attempt to optimize performance with an online evaluation of correct answer likelihood. Thus, an ongoing tally of answers for each category recorded influences later responses (e.g., Staddon et al., 1980; Wiegersma, 1982). If during the learning phase, a participant read $50 \%$ of the items from category A and 50\% from category B, answers during the recognition memory test will be tuned toward these base-rates. Early A responding will increase the likelihood of later B responses to be roughly proportional with A. These metacognitive guessing strategies pose problems for MPT models that use only response outcomes to distinguish memory processes from guessing processes. They may artificially keep different source identification responses (and errors) at similar rates. In the case of veridical identification, presentation of equal proportions of explicitly-labeled true propositions and explicitly-labeled false propositions (i.e., a "balanced" false information paradigm), may decrease the rate difference of TasT and FasF responses as well as TasF and FasT errors. Thus, during veridical learning phase presentation, equivalent proportions of true, false, and uncertain stimulus categories may have served to homogenize conditional source identification measures and MPT parameter estimates in Nadarevic and Erdfelders' $(2013,2019)$ data. Indeed, in the condition without cognitive load, Gilbert and

\footnotetext{
${ }^{8}$ Street and Kingstone (2017) also make this assumption with their "Unsure" condition.
} 
colleagues (Study 1, 1990) showed minimal rate difference between TasT (55\%) and FasF (55\%) responses as well as TasF (22\%) and FasT (21\%) errors on the original Hopi Language Task. These similar rates tend to produce equivalent probabilities of detecting the correct source of a proposition (e.g., $d_{\text {true }} \sim d_{\text {false }}$ ) in 2 HT MPT models. Prima facie, this outcome would provide evidence for the Cartesian model as it argues for two separate and sequential cognitive states for correctly identifying both true and false propositions. However, narrative-based false information paradigms that present an "imbalance" between explicitly-labeled true and explicitly-labeled false propositions (during the learning phase) tend to produce greater differences between the critical response rates without distraction/cognitive load (Table 2, Chen, 2002; Chen \& Blanchard-Fields, 2000; Gilbert et al., 1993; Pantazi et al., 2018). Narrative-based false information paradigms may obscure learning phase base-rate information that is more easily tallied during list-based false information paradigms, as FasT error rates tend to be increased (Pantazi et al., 2018).

\section{Testing the Cartesian hypothesis of "mere representation"}

While these increased true guessing bias hypothesis data are purportedly more consistent with a Cartesian than a belief-default perspective, no anti-belief-default study directly examines the defining feature of the Cartesian model: an ability to mentally represent a naïve proposition without the imbuing of truth or falsity (Figure 1). That is, the Cartesian hypothesis of an ability to "merely" represent naïve, incoming propositions without a truth stance would necessitate a temporary compartmentalization relative to other extant mental information until an individual decides to undergo an assessment process resulting in true or false tag adherence. In this perspective, represented propositions are impotent objects which must be "empowered" into belief by an outside process (Gilbert, 1993). Propositions are ballistic (Mandelbaum, 2014) in 
belief-default models and are empowered by the processes of mental representation and comprehension. A tertiary aim of the current studies was to design a paradigm where naïve, incoming propositions would produce differential outcomes based on whether they were imbued with a truth stance or not.

An experimental design where a naïve proposition imbued with truth would elicit a certain response, but a proposition not imbued with truth would elicit a different response was required. Moreover, the design must involve the theoretical withholding of veridical assessment for the task outcome to preclude assessment interactions as a confounding factor. Investigating false alarm rates (see Table 1) while varying foil propositional information during true/false recognition memory tests (e.g., Brainerd et al., 2001; Roediger \& McDermott, 1995) potentially fulfills this requirement. A false alarm is the identification of an occurrence as old (previously seen) when it is in fact new (never seen). Specifically, a design where some foils are not related to true information in memory $\left(\mathrm{NS}^{\varnothing}\right)$, some foils are similar to true information in memory but not mutually exclusive with it $\left(\mathrm{NS}^{\sim} \mathrm{t}\right)$, and some foils are similar to true information in memory and mutually exclusive with it $\left(\mathrm{NS}^{\ddagger t}\right)$, should produce divergent response outcomes. For instance, if the true, extant proposition in an individual's memory is Drew plays ice hockey on Tuesday nights a NS ${ }^{\varnothing}$ may be Drew works at an insurance company, a $\mathrm{NS}^{\sim \mathrm{t}}$ may be Drew plays basketball on Saturday nights, and a $\mathrm{NS}^{\neq \mathrm{t}}$ may be Drew plays softball on Tuesday nights. The proposition Drew plays softball on Tuesday nights has high mutually exclusivity information relative to the true knowledge that Drew, in fact, plays ice hockey Tuesday nights because of the background assumption that playing one of these games will consume much time of a weeknight. Therefore, it is unlikely that Drew would be able to regularly play both an ice hockey and softball game on Tuesday nights. The proposition Drew plays basketball on Saturday nights has 
low mutually exclusivity information relative to the true knowledge that Drew plays ice hockey on Tuesday nights because the background assumption of weeknight time limitations is absent. Thus, it is possible (i.e., more likely) that Drew would be able to regularly play an ice hockey game on Tuesday nights and a basketball game on Saturday nights. Under the constraints of the Cartesian model, we reasoned that a naïve foil proposition (not imbued with truth) could elicit a memory search based on concept similarity but not elicit background assumptions activation (Rips \& Marcus, 1977) and veridical assessment based on mutual exclusivity information. That is, the implications derived from the truth of an incoming proposition should not be automatically compared to other true information in memory in the Cartesian perspective. The Cartesian model predicts that the optimal performance during the task would be a simple comparison of the naïve proposition to extant propositions in memory. Some false alarms may occur based on concept similarity however. If the Cartesian hypothesis is correct, naïve foils high in mutual exclusivity to extant true information $\left(\mathrm{NS}^{\neq t}\right)$ should have a similar pattern of false alarms as naïve foils low in mutual exclusivity to extant true information $\left(\mathrm{NS}^{\sim t}\right)$. Foils similar to true information in memory $\left(\mathrm{NS}^{\sim \mathrm{t}}\right.$ and $\mathrm{NS}^{\neq t}$ ) may elicit increased "true" false alarm rates relative to foils that are not related to true information in memory $\left(\mathrm{NS}^{\varnothing}\right)$, but not relative to each other (i.e., $\mathrm{NS}^{\neq \mathrm{t}}$ as $\mathrm{T}=\mathrm{NS}^{\sim \mathrm{t}}$ as $\mathrm{T}>\mathrm{NS} \mathrm{\Phi}^{\varnothing}$ asT). Thus, in the example described above foil propositions involving Drew playing sports on certain nights of the week (i.e., Drew plays basketball on Saturday nights, Drew plays softball on Tuesday nights) may result in increased true responding relative to non-sport related foil propositions (i.e., Drew works at an insurance company). However, most importantly, "false" false alarm rates should not differ between the foil conditions as these foils are not similar to false information in memory (i.e., $\mathrm{NS}^{\neq \mathrm{t}}$ asF $=\mathrm{NS}^{\sim \mathrm{t}}$ asF $=$ $\left.\mathrm{NS}^{\varnothing} \mathrm{asF} \sim 0\right)$. 
By contrast, in belief-default models naïve, incoming propositions are ballistic and will automatically elicit background assumptions and mutual exclusivity information (based on extant memory activation). Thus, foil propositions imbued with truth would be disrupted by previous true knowledge which should cue false responding (Asp \& Tranel, 2013). That is, foils that are mutually exclusive with true information in memory $\left(\mathrm{NS}^{\ddagger \mathrm{t}}\right)$ should elicit increased "false" false alarm rates relative to foils that are not mutually exclusive with true information in memory (i.e., $\left.\mathrm{NS}^{\ddagger \mathrm{t}} \mathrm{asF}>\mathrm{NS}^{\sim \mathrm{t}} \mathrm{asF}=\mathrm{NS}{ }^{\emptyset} \mathrm{asF} \sim 0\right)$. Here, the foil proposition mutually exclusive with true information in memory (e.g., Drew plays softball on Tuesday nights) should result in increased false responding relative to non-mutually exclusive to true information foil propositions (e.g., Drew plays basketball on Saturday nights, Drew works at an insurance company). Thus, increased false responding to foils with high mutual exclusivity information could not be false alarm acceptance of related distracters (by processes such as phantom recollection or similarity judgment, e.g., Brainerd et al., 2008). "True" false alarm rates should not differ between the foil conditions (i.e., $\mathrm{NS}^{\neq \mathrm{t}}$ as $\mathrm{T}=\mathrm{NS}^{\sim \mathrm{t}}$ asT $=\mathrm{NS}{ }^{\varnothing}$ as $\mathrm{T} \sim 0$ ). Taken together, foil propositions imbued with truth (with high mutual exclusivity information) will have an accuracy disadvantage relative to propositions not imbued with truth (with high mutual exclusivity information). This disadvantage will manifest with increased false responses as participants will tend to answer whether the incoming proposition is true or false relative to their old true information rather than whether the incoming proposition was previously seen or not. These results would suggest an automatic, involuntary assessment of veridical information and would produce a unique type of belief bias (e.g., Pennycook et al., 2013; Sa et al., 1999) where previous knowledge overrides the logic of the current problem (see also Stanovich, 2003).

\section{Current Investigations}


In sum, there are several reasons to doubt the increased guessing true bias hypothesis currently challenging belief-default models including unfounded assumptions, reliance on negative evidence, and other circumstantial evidence consistent with the belief-default perspective. However, in the present research we sought to examine these assumptions in detail and directly test the belief-fixation models. Given a method using foil propositions during true/false recognition memory tests to offer positive evidence for the truth stance of naïve, incoming propositions (described above) we constructed an imbalanced, narrative-based false information paradigm that would 1) probe "true" and "false" false alarm rates to foils that vary in their mutual exclusivity information and 2) obscure base-rate information to minimize metacognitive guessing strategies. The establishment of our imbalanced, narrative-based false information paradigm (i.e., the Dispositional Judgment Task, DJT; Studies 1 and 2), then allowed an appraisal of the efficacy of MPT models for adjudicating belief-fixation cognitive architecture (Study 3). Finally, we sought to 1) examine age-related increases in credulity with a comparison of developmental populations in our false information paradigms (Study 3) and 2) to explore whether affective processes and related visceral autonomic feedback correlates may delineate veridical identification in accordance with the FTT (Studies 3 and 4). Data and analyses for all studies may be found here: osf.io/2rp5q/.

\section{$\underline{\text { Study } 1}$}

To meet these goals we constructed a modified version of Chen's (2002) narrative-based false information paradigm, i.e., the Dispositional Judgment Task (DJT) which includes a unique true/false recognition memory test (see also Gilbert et al., 1993). The primary aim was to determine whether an MPT model based on belief-default cognitive architecture would fit the narrative-based DJT recognition memory test data better than the a Cartesian 2HT MPT model. 
Standard 2HT, three-source variant MPT models have necessary assumption constraints that must be imposed for positive degrees of freedom and a mathematically-identifiable model with unique maximum likelihood estimators (Batchelder \& Riefer, 1990; Meiser \& Broder, 2002). To broaden our degrees of freedom and to achieve our second aim of the study, we varied the content of the foils during the true/false recognition memory test. This enabled the construction of a mathematically-identifiable six-source variant MPT model (Figure 2b) with paths for true propositions $(\mathrm{T})$, false propositions $(\mathrm{F})$, foil propositions not related to true nor false propositions $\left(\mathrm{NS}^{\varnothing}\right)$, foil propositions similar to true propositions but not mutually exclusive with them $\left(\mathrm{NS}^{\sim \mathrm{t}}\right)$, foil propositions similar to true propositions and mutually exclusive with them $\left(\mathrm{NS}^{\neq \mathrm{t}}\right)$, and foil propositions similar to false propositions $\left(\mathrm{NS}^{\sim f}\right)$.

\section{Method}

Overview. Participants read four stories regarding unique protagonists that contained both explicitly-labeled true and explicitly-labeled false statements. The stories centered around four (one per story) different protagonists with differing traits assessed: Mike, John, Brad, and Tom. If believed, the false statements were designed to sway trait judgments (intelligence or honesty) of the protagonist in a particular direction for each story: positively for Mike/Tom and negatively for John/Brad. After reading each story, participants made trait judgments about the protagonist and then were given a true/false recognition memory test regarding the story details. Trait judgments responses, true/false recognition memory test responses, and response reaction times were recorded.

Participants. A group of 26 younger adults participated in this study. The age of the participants ranged from 23 to 49 years $(M=33.9, S D=7.1)$. Four of the participants were male 
and 22 were female ${ }^{9}$. Three participants reported some education beyond high school but not a bachelor's degree, 12 participants reported attainment of a bachelor's degree, and 11 reported attainment of an advanced degree including M.A., J.D., M.D., or Ph.D. All participants were native English speakers and were free from intellectual disability, learning disabilities, psychiatric disease, substance abuse, and dementia. Participants gave informed consent approved by the Institutional Review Board of Hamline University ${ }^{10}$. This study was not preregistered prior to the research being conducted. No participants were excluded from this study post-hoc.

Stimuli and procedure. The DJT paradigm mirrored Chen (2002); see Supplementary Materials for complete procedure and stimuli. On arriving at the laboratory and completing the informed consent process, participants were escorted to a private room where they remained for the duration of the study. Participants performed the DJT on standard Windows-based PCs running E-Prime software. Participants were given both written and spoken instructions for the DJT via an experimenter.

DJT learning phase. The stories were presented statement-by-statement on the computer screen. Each story contained 6 explicitly-labeled false statements. In Mike and Tom's stories the false statements (if believed) were designed to increase the trait judgment (intelligence and honesty, respectively). In John and Brad's stories the false statements were designed to decrease the trait judgment (honesty and intelligence, respectively). Participants were told that they would be give two kinds of information, facts and fictions: 1) Facts were details about the protagonist that had been verified to be true; and facts were presented in white typeface. 2)

\footnotetext{
${ }^{9}$ Sex imbalance was due to the fact that many participants were a parent of a child recruited for research on another unrelated project. Sex differences were not predicted nor have been observed in DJT outcomes. ${ }^{10}$ Five individuals participated as part of a pilot study at the University of lowa. These individuals gave informed consent approved by the Institutional Review Board of the University of lowa.
} 
Fictions were details that did not belong to the stories in which they appear; they were actually taken from other, unrelated stories and mixed in with the facts. The fictions were presented in red typeface. Thus, the color of the typeface was the cue indicator of either true statements (white) or false statements (red). To create an imbalance in true and false statements and limit guessing strategies using base-rate information, each story contained either 11 or 12 true statements with the 6 embedded false statements spaced throughout the story.

DJT trait judgments. Following a 30 second "pause" screen, the participants rated the protagonists on 39 -point Likert scales. The first two ratings represented two dimensions relating to the trait of interest (i.e., either a cognitive assessment -- intelligence and cleverness -- for Mike and Brad, or a moral assessment -- honesty and sincerity -- for John and Tom). The final rating was a likeability scale to examine the overall evaluation of each protagonists.

DJT true/false recognition memory test. After the judgment section a true/false recognition memory test was given for each story, where participants were presented with 24 statements per story (presented in gray text on a black background) in a pseudorandom order (Supplementary Materials). For each story, 6 true statements, 6 false statements, and 12 foils were evaluated during each recognition memory test. Thus, a total of 24 true statements, 24 false statements, and 48 foils were assessed per participant in the DJT. Participants classified each statement as either 1) a fact, a true statement that was previously presented in white typeface (T); 2) a fiction, a false statement that was previously presented in red typeface (F); or 3) a foil, a never seen statement that had not been presented before (NS).

To investigate false alarm rates in accordance to our second aim, we varied how the 48 foil statements related to true and false information presented during the learning phase of the DJT (Supplementary Materials). All of the foil statements contained details that were not 
present in the learning phase. Twelve foil statements contained biographic details regarding the protagonist but were not similar to either true or false information $\left(\mathrm{NS}^{\varnothing}\right)$; e.g., a true idea from the learning phase is Mike likes watching sports on television and a $\mathrm{NS}^{\varnothing}$ is Mike fixed his own garbage disposal. Ten foil statements were similar to true information and contained low mutually exclusivity information with it $\left(\mathrm{NS}^{\sim} \mathrm{t}\right)$; e.g., a true idea from the learning phase is $\mathrm{Brad}$ enjoys playing poker with his friends and a $\mathrm{NS}^{-\mathrm{t}}$ is Brad likes playing board games with his friends. Fourteen foil statements were similar to true information and contained high mutual exclusivity information with it $\left(\mathrm{NS}^{\ddagger t}\right)$; e.g., a true idea from the learning phase is Brad often eats lunch alone and a $\mathrm{NS}^{\ddagger \mathrm{t}}$ is Brad often eats lunch with his brother. Finally, twelve foil statements were similar to false information ( $\left.\mathrm{NS}^{-\mathrm{f}}\right)$; e.g., a false idea from the learning phase is Tom helped a man fix his tire on the roadside and a $\mathrm{NS}^{\sim \mathrm{f}}$ is Tom gave a man directions on the roadside. Foil statements that were similar to false information were included to examine the likelihood of phantom recollection or similarity judgment processes with remembered false information.

Power considerations. During the creation of the DJT we sought to reduce measurement error relative to other false information paradigms as the instrument was intended for use in smaller samples (i.e., in limited neuropsychological and neuropsychiatric populations). The critical difference in veridical errors (FasT vs. TasF) during narrative-based false information paradigms tend to produce medium to large effect sizes $(d=.52-.77)$ under the condition of full attention (Chen, 2002; Chen \& Blanchard-Fields, 2000; Pantazi et al., 2018). Given Chen's (2002) original design, an alpha level of .05, and an effect size of .77, replication of these two measurements with .80 power yielded an estimate of 12 participants (Faul et al., 2007). Nonetheless, we increased the critical true/false recognition task items with the addition of the Brad and Tom vignettes relative to Chen's (2002) design. While each participant evaluated a 
total of 8 true statements and 14 false statements in Chen's (2002) true/false recognition memory test design, a participant undergoing the DJT evaluated a total of 24 true statements and 24 false statements ( 6 true and 6 false per story). In addition, our use of mixed-effects models enabled stimuli items and participants to be included as random effects, which also maximized power (see statistical approaches below). This technique reduces the chance of Type I errors compared to traditional ANOVAS (Judd et al., 2012).

Study 1 hypotheses. Hypothesis 1) In accordance with prior narrative-based false information paradigms without resource depletion (see Table 2), we hypothesized an increased FasT error rate relative to the TasF error rate. Valid belief-default model MPT parameters should reflect and be consistent with this difference. Hypothesis 2) If belief-default models are correct, then participants should have increased $\mathrm{NS}^{\neq \mathrm{t}}$ asF error rates relative to $\mathrm{NS}^{\sim \mathrm{t}}$ asF or NS ${ }^{\varnothing}$ asF errors. In addition, belief-default model MPT parameters should also reflect and be consistent with this difference. Hypothesis 3) In accordance with prior studies (e.g., Gilbert et al., 1993) we hypothesized that FasF responses would result in a longer latency than TasT responses (see Supplementary Materials for reaction time analyses). Hypothesis 4) Finally, an MPT model based on belief-default cognitive architecture should fit true/false recognition memory test data from the DJT (i.e., an imbalanced, narrative-based false information paradigm) better than standard 2HT MPT models with Cartesian assumptions.

Statistical analyses. For all tests, $\alpha=.05$. Effect sizes are reported on pairwise comparisons based on Hedges' formula of Cohen's $d$, to circumvent overestimates from the repeated measures (e.g., Dechene et al., 2010). Outliers for the judgment and reaction times analyses (Supplementary Materials) were excluded based on the median absolute deviation with a constant of 3 (Leys et al., 2013). Given the possibility of distinct patterns of false alarm rates 
based on our different foil conditions, we analyzed old (previously presented as true or false in the learning phase) and foil (never seen, or new, statements not present in learning phase) statements separately. Thus, old and foil statements were modeled with two generalized linear mixed-effects models for binomial data using the logit link target option in SPSS (for a similar statistical approach in narrative-based false information paradigms, see Pantazi et al., 2018; Quene \& van den Bergh, 2008). As memory outcomes were analyzed via linear mixed-effects models for binomial data, no outliers were excluded from the recognition memory tests results. See Supplementary Materials for reaction time statistical approaches and data analyses.

Old statements statistics. For the old statements mixed-effects model, we designated the three possible responses as repeated measures at three different levels of the fixed factor of classification (hit, TasT or FasF; vs. veridical error, TasF or FasT; vs. miss, TasNS or FasNS) on the target binomial variable. That is, the target variable was repeated three times for each of the 48 items participants answered during the recognition memory tests. The target variable was coded 1 at the level of classification factor that represented participants' response, and 0 at the other two levels. Our fixed factors for the old statements mixed-effects model included classification and statements; the latter reflecting whether each statement was presented as true or false during the learning phases of each story. We examined hypothesis 1 with the inclusion of a classification by statements interaction, which allowed a within-subjects distinction in the memory outcomes for true and false statements. Intercepts and slopes for participants and recognition memory test items were added as random effects.

$\underline{\text { Foil statements statistics. }}$. We ran an analogous model on foil statements with statements $\left(N S^{\emptyset}, N S^{\sim t}, N S^{\ddagger t}, N S^{\sim}\right.$ ) and answer (correct rejection, "true" false alarm, "false” false alarm) as fixed factors. Hypothesis 2 was examined with the inclusion of an answer by statements 
interaction. Again, intercepts and slopes for participants and recognition memory test items were added as random effects.

Trait judgment statistics. The three trait judgments for each protagonist were analyzed with a mixed-effects model. Fixed factors included false valence (positive falses, negative falses) and critical trait (cognitive assessment, moral assessment, likeable assessment) which enabled a false valence by critical trait interaction for an examination of whether the valence of the explicitly-labeled false statements deviated trait judgments under full attention. Intercepts for participants were added as random effects.

Judgment-memory relationship statistics. To investigate potential relationships between the trait judgments and FasT error rates a Pearson correlation was conducted with the whole sample (Supplementary Materials).

Multinomial processing tree statistics. MPT models used to analyze true/false recognition memory test results often consist of separate processing trees for old statements presented as true, old statements presented as false, and new (or foil) statements never seen before. Putatively, each branch of a tree represents a possible sequence of cognitive processes resulting in the potential participant responses true, false, or never seen. Parameters in the model reflect transition probabilities. Traditional source memory parameters include: $D=$ probability of statement recognition or foil detection, $d=$ probability of correctly discriminating the source (i.e., in this case true or false), $b=$ probability of guessing "old" to items not recognized nor identified as a foil, $g=$ probability of guessing that a non-detected item belongs to a particular source, and $a=$ probability of guessing that a detected but non-discriminated item belongs to a particular source. Our MPT model with Cartesian assumptions (Figure 2a) was derived from the 2HT, three-source MPT models used by Nadarevic and Erdfelder $(2013,2019)$ without the three 
variants of the guessing biases $a$ and $g$ (see also, Riefer et al., 1994). Two points are of note in the Cartesian assumption MPT model: 1) $d_{t r u e}$ indicates the probability of correctly identifying recognized statements as true. In the Cartesian belief model (Figure 1) this is equivalent to the probability of identifying whether a proposition has a true tag associated with it. 2) The foil statements information content conditions were collapsed into a single foil statement processing tree as the Cartesian belief model does not predict increased false responses to foils with high mutual exclusivity information. To preserve degrees of freedom and make the MPT model with Cartesian assumptions mathematically-identifiable, we placed theoretically motivated constraints on the parameters. Specifically, we echoed Nadarevic and Erdfelders' (2013) constraints of recognition parameter equality $\left(D_{\text {true }}=D_{\text {false }}=D_{N S}\right)$ and guessing true parameter equality $\left(a_{\text {true }}=\right.$ $\left.g_{\text {true }}\right)$. Parameters $d_{\text {true }}$ and $d_{\text {false }}$ were free to vary as no constraints were placed on them.

By contrast, our MPT model with belief-default assumptions does not include a $d_{t r u e}$ parameter (Figure 2b). Propositions recognized without tags associated with them are identified as true. Moreover, in the false statement processing tree a $d_{\text {false }}$ parameter indicates the probability of correctly identifying a recognized statement as false. This is equivalent to the probability of identifying whether a proposition has a false tag associated with it. The imbalance between the one-stage correct identification of true propositions $\left(D_{\text {true }}\right)$ and the two-stage correct identification of false propositions ( $D_{\text {false }}$ and $d_{\text {false }}$ ) is supported by our reaction time results as TasT responses were quicker than FasF responses (see also, Gilbert et al., 1990). Critically, the false statement processing tree does not include a guessing parameter $a$. If a false tag for a recognized proposition is not identified it will be considered true (i.e., a FasT error) with the probability $1-d_{\text {false }}$. A comparable veridical error outcome does not exist in the true statement processing tree (i.e., TasF error). Thus, the structure of our MPT model with belief-default 
assumptions posits that increased FasT errors relative to TasF errors are due to fundamental processing differences rather than a post-hoc guessing strategy (e.g., Street \& Kingstone, 2017). Contrary to Cartesian belief models, belief-default models predict differing rates of NSasF errors as a function of foil information. Thus, in our MPT model with belief-default assumptions, we included a new parameter $z$ which captures the probability that a foil statement related to prior knowledge is misidentified as false (i.e., $z \emptyset, z_{\sim t}, z_{\neq t}$, and $z_{\sim f}$, respectively; Figure $2 \mathrm{~b}$ ). The parameter $n$ specifies the probability that a foil statement related to prior knowledge not misidentified as false is correctly identified as never seen. Additionally, parameter $s$ was included in the $\mathrm{NS}^{\sim \mathrm{t}}$ and $\mathrm{NS}^{\sim \mathrm{f}}$ processing trees to capture false alarms due to processes such as phantom recollection or similarity judgment (i.e., $s_{\text {true }}$ and $s_{f a l s e}$, respectively; Figure $2 \mathrm{~b}$ ). To make the MPT model with belief-default assumptions mathematically-identifiable, we placed two theoretically motivated constraints on the parameters: 1) the guessing true parameter $g_{\text {true }}$ was kept constant at .50, and 2) the "false" false alarm parameter in the foil statement processing tree not related to true nor false information, $z \varnothing$, was equated to the "false" false alarm parameter in the foil statement processing tree similar to true information but not mutually exclusive with it, $z \sim$ t. Neither $\mathrm{NS}^{\varnothing}$ nor $\mathrm{NS}^{\sim \mathrm{t}}$ were predicted to elicit increased false responding and thus should theoretically be comparable. Recognition parameters $D_{\text {true }}, D_{\text {false }}$, and $D_{N S}$ were free to vary as no constraints were placed on them ${ }^{11}$.

MPT analyses were analyzed using the computer program multitree (Moshagen, 2010), which minimizes a $\mathrm{G}^{2}$ statistic (see Supplementary Materials for equations and raw MPT data).

\footnotetext{
${ }^{11}$ We conducted an additional analysis with Studies 1-3 DJT data on a "hybrid" MPT model that used a Cartesian true and false source recognition and source discrimination parameter design and a belief-default foil parameter design. While the Cartesian model does not predict increased false responses to foils that vary in their mutual exclusivity to true information, the critical differences between the models are how the old true and false propositions are adjudicated. On the whole, the "hybrid" MPT model fit the DJT data from Studies 1-3 poorly (see Supplementary Materials for the full analyses).
} 
Distinct from conventional statistics, a lower $\mathrm{G}^{2}$ and $p$ values greater than .05 indicate a better overall model fit. We contrasted the fit of the recognition memory test results to an MPT model with Cartesian assumptions and an MPT model with belief-default assumptions (Figure 2) to address hypothesis 4 . No outliers were excluded from the MPT analyses.

\section{Results}

Old statements results. There was a main effect of classification, $F(2,3738)=734.47, p$ $<.001$. Old statements were more likely to be hits than veridical errors, $t(3738)=70.39, p<$ $.001, d=2.95,95 \%$ CI $[2.83,3.06]$, or misses, $t(3738)=68.14, p<.001, d=2.81,95 \%$ CI [2.70, 2.92]. In addition, there was a classification by statements interaction, $F(2,3738)=12.19, p<$ .001. True statements were more correctly classified as true (TasT) than false statements correctly classified as false $(\mathrm{FasF}), t(3738)=3.46, p=.001, d=.21,95 \%$ CI $[.10, .32]$. Moreover, in support of hypothesis 1 , false statements were more likely misclassified as true (FasT) than true statements were misclassified as false (TasF), $t(3738)=3.74, p<.001, d=.31$, 95\% CI [.20, .42] (Figure 3a). No significant differences were observed between true and false statements misclassified as never seen (TasNS FasNS).

Foil statements results. A subsequent mixed-effects model for foil statements found a main effect of answer, $F(2,3732)=574.13, p<.001$. Foil statements were more correctly identified as never seen (NSasNS) than misclassified as true (NSasT), $t(3732)=58.54, p<.001$, $d=2.47,95 \%$ CI $[2.37,2.58]$ or misclassified as false $(\mathrm{NSasF}), t(3732)=43.14, p<.001, d=$ $1.64,95 \%$ CI $[1.55,1.73]$. Moreover, foil statements were more likely to be misclassified as false (NSasF) than misclassified as true (NSasT), $t(3732)=6.49, p<.001, d=.45,95 \%$ CI $[.37$, .53]. Importantly, an answer by statements interaction, $F(6,3732)=30.06, p<.001$, indicated that the foil statements content altered responding. Specifically, foil statements not related to 
true nor false statements were more correctly rejected (NS ${ }^{\left.a_{a s N S}\right)}$ than foil statements similar but not mutually exclusive to true statements ( $\mathrm{NS}^{\sim \mathrm{t}}$ asNS), $t(3732)=2.31, p=.021, d=.23,95 \% \mathrm{CI}$ $[.07, .40]$, foil statements similar and mutually exclusive to true statements $\left(\mathrm{NS}^{\neq \mathrm{t}}\right.$ asNS), $t(3732)=$ $10.26, p<.001, d=.79,95 \%$ CI $[.63, .94]$, or foil statements similar to false statements $\left(\mathrm{NS}^{\sim \mathrm{f}}\right.$ asNS $), t(3732)=5.99, p<.001, d=.51,95 \% \mathrm{CI}[.35, .67] . \mathrm{NS}^{\sim \mathrm{t}}$ asNS responses were more likely than $\mathrm{NS}^{\neq \mathrm{t}}$ asNS responses, $t(3732)=7.37, p<.001, d=.56,95 \%$ CI $[.40, .73]$, and $\mathrm{NS}^{\sim \mathrm{f}}$ asNS responses, $t(3732)=3.47, p=.001, d=.29,95 \%$ CI [.12, .45]. Additionally, $\mathrm{NS}^{\sim \mathrm{f}}$ asNS responses were more likely than $\mathrm{NS}^{\neq \mathrm{t}}$ asNS responses, $t(3732)=3.74, p<.001, d=$ $.29,95 \%$ CI $[.13, .44]$. No significant differences were found between the differing foil statements misclassified as true $\left(\mathrm{NS}^{\varnothing}{ } \mathrm{asT} \sim \mathrm{NS}^{\sim \mathrm{t}}\right.$ as $\mathrm{T} \sim \mathrm{NS}^{\ddagger \mathrm{t}}$ as $\mathrm{T} \sim \mathrm{NS}^{\sim \mathrm{f}}$ as $\left.\mathrm{T}\right)$. In support of hypothesis 2 , foil statements similar and mutually exclusive to true statements were more likely classified as false (NS ${ }^{\neq t}$ asF) than foil statements not related to true nor false statements $\left(\mathrm{NS}^{\emptyset_{\mathrm{asF}}}\right), t(3732)=9.94, p<.001, d=.78,95 \%$ CI $[.63, .94]$, foil statements similar but not mutually exclusive to true statements $\left(\mathrm{NS}^{\sim \mathrm{t}} \mathrm{asF}\right), t(3732)=8.07, p<.001, d=.62,95 \% \mathrm{CI}[.45$, $.78]$, and even foil statements similar to false statements $\left(\mathrm{NS}^{\sim \mathrm{f}} \mathrm{asF}\right), t(3732)=4.02, p<.001, d=$ $.30,95 \%$ CI $[.15, .46]$ (Figure 3a). Finally, $\mathrm{NS}^{\sim \mathrm{f}}$ asF errors were more likely than $\mathrm{NS}^{\varnothing}$ asF errors, $t(3732)=5.51, p<.001, d=.50,95 \%$ CI $[.34, .66]$, and $\mathrm{NS}^{\sim \mathrm{t}}$ asF errors, $t(3732)=3.85, p<.001$, $d=.33,95 \%$ CI $[.16, .49]$.

Trait judgments results. Of the total responses, only 1 data point $(.32 \%)$ was deemed an outlier and was excluded. There was a main effect of false valence, $F(1,280.03)=15.68, p<$ .001. However, pairwise comparisons indicated that negative falses had increased ratings relative to positive falses, $M D=.46, p<.001, d=.34,95 \%$ CI $[.11, .56]$ (Figure 3b). Thus, the data show that participants were using the falsification cues to moderate their trait judgments. 
Explicitly-labeled false information was remembered at the time of the judgments and may have acted to produce an "over-compensation effect" as knowledge of false information about a protagonist in a domain resulted in more opposing judgments. For example, remembering that some false information about John was negative in the moral dimension tended to produce higher moral trait judgments of John than if the false information was not present (for a similar effect in younger adults, see Chen, 2002). There also was a main effect of critical trait, $F(2,280.03)=$ $6.16, p=.002$. Pairwise comparisons indicated that cognitive assessments (intelligent/clever) had reduced ratings relative to likeable assessments, $M D=-.49, p=.002, d=.39,95 \% \mathrm{CI}[.11$, .66]. Moreover, our model did indicate a false valence by critical trait interaction, $F(2,280.03)$ $=9.68, p<.001$. Pairwise comparisons revealed that negative falses had increased ratings relative to positive falses in moral assessments specifically, $M D=1.11, p<.001, d=.80,95 \%$ CI $[.40,1.21]$, in accordance with an "over-compensation effect". However, taken together, there was no evidence that the valence of the false statements in the stories swayed judgments in the designed direction (i.e., increased trait ratings for positive falses and decreased trait ratings for negative falses).

Judgment-memory relationship results. We did not observe a significant relationship between FasT errors and critical trait judgments, $r(26)=.11, p=.59$.

Multinomial processing tree results. The MPT model with Cartesian assumptions fit the data poorly, $\mathrm{G}^{2}(1)=110.49, p<.001$. Of note, the parameter $d_{\text {true }}$ was estimated at 1.00 ; an indicant that when true statements are recognized they are considered true without an additional source identification step. In support of hypothesis 4, our MPT model with belief-default assumptions fit the data well, $\mathrm{G}^{2}(1)=1.87, p=.172$ (see Supplementary Materials for the raw data used). As shown in Table 3, the parameter estimates for the MPT model with belief-default 
assumptions correspond to the main effects reported in the old and foil statements mixed-effects models. True and false statements were recognized with the same probability $\left(D_{\text {true }}=.89, D_{\text {false }}\right.$ $=.89)$. Correct false statement identification included an additional process to which participants were successful for most items $\left(d_{\text {false }}=.92\right)$. However, of the false statements correctly recognized, $8 \%$ of the items were mistaken as true (1-dfalse resulting in a FasT error. Thus, in support of hypothesis 1, there was a substantially greater rate of FasT errors relative to TasF errors due to the model's structure. Constraining $D$ to be equal for true, false, and foil statements led to a good model fit, $\Delta \mathrm{G}(2)=.03, p=.985$, suggesting that $D$ did not significantly differ between the conditions. Moreover, as evidence toward hypothesis 2, the "false" false alarm parameter for foil statements with high mutual exclusivity to true information $(z \neq t)$ was greater relative to "false" false alarm parameter for foil statements with low mutual exclusivity to true information $\left(z \emptyset, z_{\sim}\right), \Delta \mathrm{G}(1)=123.77, p<.001$. Remarkably, $z \neq t$ was even greater than the "false" false alarm parameter for foil statements that were similar to false information $\left(z_{\sim f}\right)$, $\Delta \mathrm{G}(1)=16.15, p<.001$. However, $z_{\sim f}$ was greater than $z_{\emptyset}$ and $z_{\sim t}, \Delta \mathrm{G}(1)=25.04, p<.001$. Similarity parameters to true and false statements ( $s_{\text {true }}, s_{\text {false }}$, respectively) were highly variable in our model (see Table 3), likely as a result of relatively few outcomes. Constraining $s_{\text {true }}$ and $s_{\text {false }}$ to be equal led to a good model fit, $\Delta \mathrm{G}(1)=.03, p=.862$.

\section{Discussion}

Study 1 constructed an imbalanced, narrative-based false information paradigm and examined Cartesian and belief-default model implications with mixed-effects and multinomial processing tree statistical models. Broadly, it offers two lines of evidence in favor of beliefdefault models. 
First, this study indicates that when a naïve proposition is mentally represented, the implications derived from its truth are automatically compared to extant true mental information. When participants mentally represented foil statements that were similar and mutually exclusive to extant true mental information, they often produced an incorrect false response rather than the correct never seen response $\left(\mathrm{NS}^{\ddagger \mathrm{t}}\right.$ asF errors, Figure $\left.3 \mathrm{a}\right)$. In effect, they were mistakenly answering whether or not the proposition was true or false relative to their current beliefs rather than whether the proposition was previously seen or not. When participants mentally represented foil statements that were similar but not mutually exclusive to extant true mental information, they did not have an increased incorrect false response rate ( $\mathrm{NS}^{\sim \mathrm{t}}$ asF errors). Moreover, even when participants mentally represented foil statements that were similar to false mental information, they did not produce as many incorrect false responses ( $\mathrm{NS}^{\sim \mathrm{f}}$ asF errors) as to foil statements that were mutually exclusive to true mental information. Thus, in true/false recognition memory tests, when foil information is mutually exclusive with previously learned true information, participants produce a "false" false alarm belief bias (FFABB), where previous knowledge overrides the logic of the memory task (for a general review of belief biases, see Stanovich, 2003). To our knowledge this is the first study to describe and offer an explanation for this effect, although see Pantazi et al. (2018) for a familiarity-based explanation of new statements misclassified as fals $e^{12}$. Individuals cannot withhold truth implications for optimal memory task performance. This inability is discrepant with the Cartesian belief model's view of the capacity to represent propositions without a true or false stance, and supports the beliefdefault model view of ballistic propositional representation.

\footnotetext{
12 Unkelbach and Rom (2017) also offer a fluency-based/coherence explanation of false responding to contradictory statements of previously-seen true statements. Many of these contradictory statements have high mutual exclusivity content (Appendix A, Unkelbach \& Rom, 2017) relative to their surrogate true statements. See also Garcia-Marques et al., (2015) for the time-dependent nature of these falsification processes.
} 
Second, our cognitive architecture-derived belief-default MPT model fits true/false recognition memory test data from narrative-based false information paradigms better than the MPT model with Cartesian assumptions. Specifically, a belief-default MPT model design that lacks a source identification process for true statements (i.e., $d_{\text {true }}$ ) and produces a FasT error as the result of a failed source identification for false statements (i.e., $1-d_{\text {false }}$ ) was well-matched to our true/false recognition memory test data (Figure $2 b$ ). Thus, under conditions where base-rate information is more obscure, the truth bias is exaggerated (Pantazi et al., 2018), veridical errors are imbalanced (i.e., FasT > TasF errors; Table 2; Figure 3a), and MPT models with beliefdefault assumptions correspond well to the data. While MPT models putatively disentangle memory processes from guessing processes, our evidence suggests these results and, more broadly, belief model MPT conclusions generally, rely on cognitive process assumptions and the type of false information paradigm design employed.

It is important to note that belief-default MPT model parameter estimations are consistent with the critical interaction effects examined with our mixed-effects models. For instance, fundamental processing differences (reflected in the belief-default MPT model) between true and false statements predict a greater prevalence of FasT errors relative to TasF errors and a quicker reaction time of TasT responses relative to FasF responses (see Supplementary Materials for the complete reaction time analyses; Figure 2). Our data confirmed both of these hypotheses (Figure 3a). Moreover, greater incorrect false responding to foil statements that were mutually exclusive to true information $\left(\mathrm{NS}^{\neq \mathrm{t}} \mathrm{asF}\right)$ relative to other "false" false alarms were reflected in parameter estimates of the belief-default MPT model (i.e., $z_{\neq t}>z_{\emptyset}, z_{\sim t}$, and $z_{\sim f}$ ). While the overall MPT model fit the data well, similarity parameters $\left(s_{\text {true }}, S_{\text {false }}\right)$ did not achieve reliable estimates based 
on a low number of outcomes. A replication of this design was warranted with an increased power to further explore the validity of these parameters.

Beyond the recognition memory results, there was evidence that our participants appropriately used the falsification cues when forming their trait judgments of the protagonists (see also Chen, 2002). That is, they were not influenced in their judgments by the explicitlylabeled false information. This is discrepant with some narrative-based false information paradigm research that has shown false influences to judgments in individuals under full attention (e.g., Pantazi et al., 2018). Content differences between the narrative-based false information designs may explain this disparity.

To validate and extend the central findings reported here, we sought to replicate this design with distinct participants. In addition, during our initial data exploration of Study 1, we observed some FasT error differences between the 4 stories. To examine if these differences were due to the content of the stories themselves or a potential order effect, we planned to vary which stories were presented first between participants in our second study.

\section{Study 2}

To confirm the basic results of Study 1, we replicated our design with a set of new participants. Here, we varied which participants received either Mike and Johns' stories or Brad and Toms' stories initially to examine potential order effects of the DJT. This enabled a potential between-subject difference we examined with our mixed-effects models on the true/false recognition memory test results.

\section{Method}

Overview. As with Study 1, participants performed the DJT. 
Participants. A group of 34 individuals participated in this study ${ }^{13}$. The age of the participants ranged from 18 to 21 years $(M=19.1, S D=1.0)$. Twenty-two of the participants were male and 12 were female ${ }^{14}$. All participants were undergraduates at an elite midwestern university. All participants were free from intellectual disability, learning disabilities, and dementia. Participants gave informed consent approved by the Institutional Review Board at the University of Norte Dame. This study was not pre-registered prior to the research being conducted. No participants were excluded from this study post-hoc.

Stimuli and procedure. The procedure and stimuli were identical to that of Study 1 with the exception that 15 participants received Mike and Johns' portions of the task prior to Brad and Toms' portion, and 19 participants received Brad and Tom' portions prior to Mike and Johns' portions.

Study 2 hypotheses. The hypotheses were identical to Study 1: 1) FasT errors would be greater than TasF errors, 2) $\mathrm{NS}^{\neq \mathrm{t}}$ asF false alarms would be greater than $\mathrm{NS}^{\sim \mathrm{t}}$ asF or $\mathrm{NS}^{\varnothing}{ }$ asF false alarms, 3) FasF responses would have longer reaction times than TasT responses (see Supplementary Materials for the reaction time analyses), and 4) the belief-default MPT model would fit the data better than the MPT model with Cartesian assumptions.

Statistical analyses. Statistical analyses were also identical to Study 1 with the exception that we included an additional group-level fixed factor in our old and foil statements mixedeffects analyses. Thus, the fixed factors for the old and foil statements mixed-effects models included classification/answer, statements, and group; the latter reflecting whether the

\footnotetext{
${ }^{13}$ This was a control group of an experimental manipulation examining induced stress, credulity, and false memory. Twenty-nine individuals constituted the experimental group which is the focus of a separate study. The data of the experimental group were not analyzed at the time of this manuscript preparation. ${ }^{14}$ Again, sex differences were a byproduct of participant recruitment strategies. No sex differences were predicted nor observed in the DJT outcomes.
} 
participant received Mike/Johns' stories initially or Brad/Toms'. All two- and three-way interactions of the three fixed factors were examined in each model.

Results

Old statements results. There was a main effect of classification, $F(2,4884)=918.94, p$ $<.001$. Old statements were more likely to be hits than veridical errors, $t(4884)=72.49, p<$ $.001, d=2.66,95 \%$ CI $[2.57,2.76]$, or misses, $t(4884)=65.79, p<.001, d=2.35,95 \%$ CI [2.26, 2.44]. In addition, misses were more likely than veridical errors, $t(4884)=3.48, p=.001, d=$ $.14,95 \%$ CI $[.07, .21]$. There was also a classification by statements interaction, $F(2,4884)=$ $16.79, p<.001$. True statements were more correctly classified as true (TasT) than false statements correctly classified as false (FasF), $t(4884)=3.76, p<.001, d=.18,95 \%$ CI [.09, .28]. Similar to the evidence toward Study 1, our analysis of data in Study 2, revealed that, again, hypothesis 1 was supported as false statements were more likely misclassified as true (FasT) than true statements were misclassified was false (TasF), $t(4884)=4.69, p<.001, d=$ $.33,95 \%$ CI $[.23, .43]$ (Figure 4a). No significant differences were observed between true and false statements misclassified as never seen (TasNS $\sim$ FasNS). While there was no main effect of group, there was a three-way interaction observed between classification, statements, and group, $F(2,4884)=3.16, p=.043$. Participants that received the Brad/Tom portion of the DJT first were more likely to correctly classify false statements as false (FasF) relative to participants that received the Mike/John portion of the DJT first, $t(4884)=2.40, p=.017, d=.17,95 \% \mathrm{CI}$ $[.03, .31]$. Thus, there was some evidence for an order effect in the DJT. However, given that the critical FasT error rate did not significantly differ between the groups ${ }^{15}$, order-related group effects were not analyzed in the trait judgment, reaction time, and MPT analyses.

\footnotetext{
${ }^{15}$ In addition, TasT, TasF, TasNS, and FasNS response rates did not significantly differ between the groups.
} 
Foil statements results. A subsequent mixed effects model for foil statements found a main effect of answer, $F(2,4872)=822.01, p<.001$. Foil statements were more correctly identified as never seen (NSasNS) than misclassified as true (NSasT), $t(4872)=76.22, p<.001$, $d=2.91,95 \%$ CI $[2.81,3.00]$ or misclassified as false (NSasF), $t(4872)=59.13, p<.001, d=$ $2.08,95 \% \mathrm{CI}[2.00,2.17]$. Foil statements were more likely to be misclassified as false (NSasF) than misclassified as true (NSasT), $t(4872)=6.66, p<.001, d=.37,95 \%$ CI $[.30, .44]$. In addition, an answer by statements interaction, $F(6,4872)=13.50, p<.001$, provided evidence that foil statement content altered responding, in correspondence with Study 1. Foil statements not related to true nor false statements were more correctly rejected (NS ${ }^{\varnothing}$ asNS) than foil statements similar but not mutually exclusive to true statements ( $\left.\mathrm{NS}^{\sim \text { tasNS}}\right), t(4872)=2.36, p=$ $.018, d=.20,95 \% \mathrm{CI}[.06, .34]$, foil statements similar and mutually exclusive to true statements $\left(\mathrm{NS}^{\ddagger \mathrm{t}} \mathrm{aSNS}\right), t(4872)=6.87, p<.001, d=.47,95 \% \mathrm{CI}[.34, .61]$, or foil statements similar to false statements $\left(\mathrm{NS}^{\sim \mathrm{f}}\right.$ asNS), $t(4872)=4.91, p<.001, d=.37,95 \% \mathrm{CI}[.23, .51] . \mathrm{NS}^{\sim \mathrm{t}}$ asNS responses were more likely than $\mathrm{NS}^{\neq t}$ asNS responses, $t(4872)=4.10, p<.001, d=.28,95 \% \mathrm{CI}$ $[.14, .42]$, and $\mathrm{NS}^{-\mathrm{f}}$ asNS responses, $t(4872)=2.36, p=.018, d=.17,95 \%$ CI $[.03, .32]$.

However, there were no significant differences between $\mathrm{NS}^{\sim f}$ asNS and $\mathrm{NS}^{\ddagger t}$ asNS responses. In addition, no significant differences were found between the differing foil statements misclassified as true $\left(\mathrm{NS}^{\emptyset}{ } \mathrm{asT} \sim \mathrm{NS}^{\sim \mathrm{t}} \mathrm{as} \mathrm{T} \sim \mathrm{NS}^{\ddagger \mathrm{t}}\right.$ as $\mathrm{T} \sim \mathrm{NS}^{\sim f}$ asT $)$. In correspondence with Study 1 and in support of hypothesis 2 , foil statements similar and mutually exclusive to true statements were more likely classified as false $\left(\mathrm{NS}^{\ddagger t}\right.$ asF) than foil statements not related to true nor false statements $\left(\mathrm{NS}^{\varnothing} \mathrm{asF}\right), t(4872)=5.91, p<.001, d=.42,95 \% \mathrm{CI}[.29, .55]$, and foil statements similar but not mutually exclusive to true statements ( $\mathrm{NS}^{\sim \mathrm{f}}$ asF), $t(4872)=4.37, p<.001, d=.31$, 95\% CI [.17, .45] (Figure 4a). However, there were no significant differences between NS ${ }^{\ddagger t}$ asF 
and $\mathrm{NS}^{\sim \mathrm{f}}$ asF errors. $\mathrm{NS}^{\sim \mathrm{f}}$ asF errors were more likely than $\mathrm{NS}^{\varnothing}$ asF errors, $t(4872)=4.29, p<$ $.001, d=.33,95 \% \mathrm{CI}[.19, .47]$, and $\mathrm{NS}^{\sim \mathrm{t}}$ asF errors, $t(4872)=2.87, p=.004, d=.22,95 \% \mathrm{CI}$ $[.08, .37]$. There also was no evidence of an order effect in the foil statements mixed-effects model, as there was no main effect of group, and no significant two-way or three-way interaction with group.

Trait judgments results. Of the total responses, 2 data points (.49\%) were deemed outliers and were excluded. Similar to Study 1, we found a main effect of false valence, $F(1$, 367.12) $=13.14, p<.001$. Pairwise comparisons indicated an "over-compensation effect" as negative falses had increased ratings relative to positive falses, $M D=.50, p<.001, d=.31,95 \%$ CI $[.12, .51]$ (Figure $4 b)$. There also was a main effect of critical trait, $F(2,367.12)=13.68, p<$ .001. Pairwise comparisons indicated that likeable assessments had increased ratings relative to cognitive assessments, $M D=.87, p<.001, d=.54,95 \%$ CI [.30, .79], and moral assessments, $M D=.57, p=.003, d=.34,95 \% \mathrm{CI}[.10, .58]$. Finally, there was a false valence by critical trait interaction, $F(2,367.12)=6.04, p=.003$. Replicating the "over-compensation effect" found in Study 1, pairwise comparisons showed that negative falses had increased ratings relative to positive falses in moral assessments specifically, $M D=1.18, p<.001, d=.80,95 \%$ CI $[.45$, 1.15]. However, as with the Study 1 data, there was no evidence from Study 2 's data that the valence of the false statements swayed judgments in the designed direction (Figure 4b).

Judgment-memory relationship results. We observed that the more participants misremembered false statements as true (FasT errors), the larger the impact of the explicitlylabeled false statements was on their critical trait judgments ${ }^{16}, r(34)=.54, p=.001$.

\footnotetext{
${ }^{16}$ See Supplementary Materials for the trait judgment index calculation used.
} 
Multinomial processing tree results. Study 2 contrasted the same MPT models as Study 1 (Figure 2). In accordance with Study 1, the MPT model with Cartesian assumptions fit the Study 2 data poorly ${ }^{17}, \mathrm{G}^{2}(1)=95.76, p<.001$. Again, $d_{\text {true }}$ was estimated at 1.00 ; an indication that the data best fit a model where recognized true statements are never subsequently guessed true or false with probability $a_{\text {true }}$ or 1-atrue, respectively. Replicating Study 1 and in support of hypothesis 4, the MPT model with belief-default assumptions fit the data well, $\mathrm{G}^{2}(1)=1.06, p=$ .303 (see Supplementary Materials for the raw data used). The parameter estimates for the MPT model with belief-default assumptions correspond to the main effects reported in the old and foil statements mixed-effects models (Table 3$)$. Parameter estimates for recognition $\left(D_{\text {true }}, D_{\text {false }}\right.$, and $D_{N S}$ ) were comparable. Constraining $D$ to be equal for true, false and foil statements led to a good model fit, $\Delta \mathrm{G}(2)=2.01, p=.365$, suggesting that $D$ did not significantly differ between the conditions. Importantly, $d_{\text {false }}$ corresponded with Study 1 as it was estimated at .91. Thus, in Study 2, of the false statements correctly recognized, $9 \%$ of the items were mistaken as true (1$d_{\text {false }}$ ) resulting in a FasT error. Further supporting hypothesis 2 and consistent with Study 1, the "false" false alarm parameter for foil statements with high mutual exclusivity to true information $(z \neq t)$ was greater relative to "false" false alarm parameter for foil statements with low mutual exclusivity to true information $\left(z \emptyset, z_{\sim}\right), \Delta \mathrm{G}(1)=44.34, p<.001$. However, in contrast to Study $1, z \neq t$ was not significantly different than the "false" false alarm parameter for foil statements that were similar to false information $\left(z_{-f}\right), \Delta \mathrm{G}(1)=3.30, p=.069$. Parameter $z_{\sim f}$ was greater than $z \emptyset$ and $z_{\sim t}, \Delta \mathrm{G}(1)=35.83, p<.001$. As with Study 1 , similarity parameters $\left(s_{\text {true }}, s_{\text {false }}\right)$ were highly variable in our model (Table 3), most likely an outcome of relatively few data points. Constraining $s_{\text {true }}$ and $s_{\text {false }}$ to be equal led to a good model fit, $\Delta \mathrm{G}(1)=.26, p=.613$.

\footnotetext{
${ }^{17}$ See Supplementary Materials for the Study 2's DJT data applied to the "hybrid" MPT model.
} 


\section{Discussion}

Study 2 aimed to replicate Study 1's main results with a unique sample of participants and examine whether FasT error differences between stories may be the result of an order effect. While a small order effect was observed between participants that received Mike/John DJT portion first relative to participants that initially received Brad/Tom portion for FasF responses, no order effects were observed between any other true/false recognition memory outcome, including the critical FasT error. Therefore, Study 2's participants were collapsed into a single group and order effects were not examined in subsequent analyses.

In accordance with Study 1, we again observed evidence of FFABB as participants produced increased incorrect false responses to foil statements that were similar and mutually exclusive to extant true mental information $\left(\mathrm{NS}^{\neq \mathrm{t}} \mathrm{asF}\right)$ relative to foil statements that were similar but not mutually exclusive to true mental information $\left(\mathrm{NS}^{\sim \mathrm{t}} \mathrm{asF}\right)$ and foil statements not similar to extant mental information (NS ${ }^{\varnothing}$ asF; Figure 4a). In contrast to Study 1 , however, rates of $\mathrm{NS}^{\ddagger \mathrm{t}}$ asF errors were not significantly different than incorrect false responses to foil statements that were similar to false mental information $\left(\mathrm{NS}^{\sim \mathrm{f}}\right.$ asF). Overall, Study 2 produced a decreased rate of $\mathrm{NS}^{\neq \mathrm{t}}$ asF relative to Study 1 . We suggest that the difference in rates of $\mathrm{NS}^{\neq \mathrm{t}}$ asF between Study $1(M=.32)$ and $2(M=.19)$ is likely due to the differences in demographic variables of participants between the studies. The participants in Study 2 were all undergraduate college students that attended an elite academic institution while the participants in Study 1 were mainly a community-based sample of younger adults. An examination of potential age differences in FFABB was a central objective of Study 3.

Study 2 also replicated Study 1's main MPT results. Our belief-default MPT model design without a source identification process for true statements (i.e., $d_{\text {true }}$ ) and a pathway for 
FasT errors as the result of a failed source identification for false statements (i.e., 1 - $d_{\text {false }}$ ) fit the data better than our MPT model with Cartesian assumptions (Table 3; Figure 2). Of note, our belief-default MPT model's similarity parameters $\left(s_{\text {true }}, s_{\text {false }}\right)$ again did not achieve reliable estimates (Table 3) even with an increased number of participants relative to Study 1. Moreover, in contrast to Study 1, Study 2 did not find a difference between "false" false alarm parameters $z_{\neq t}$ and $z_{\sim f}$ which is consistent with the lack of difference in rates of $\mathrm{NS}^{\neq \mathrm{t}}$ asF and $\mathrm{NS}^{\sim \mathrm{f}}$ asF errors. Reaction time data from Studies 1 and 2 support the belief-default perspective (Supplementary Materials). Correctly identifying false information as false (FasF) takes longer than correctly identifying true information as true (TasT). As several other researchers have indicated (e.g., Carpenter \& Just, 1975; Gilbert, 1991), this reliable time difference may reflect additional cognitive processing for the correct identification of a false tag. Our analysis of foil statement reaction times in Study 2 did not show an interaction between answer and statements which was evident in Study 1 (Supplementary Materials). However, we did observe that on the whole foil statements similar and mutually exclusive to true statements $\left(\mathrm{NS}^{\neq t}\right)$ had longer reaction times relative to foil statements similar and not mutually exclusive to true statements $\left(\mathrm{NS}^{\sim t}\right)$ and foil statements not similar to true nor false statements $\left(\mathrm{NS}^{\varnothing}\right)$. This result is broadly consistent with the foil reaction time answer by statements results in Study 1. Taken together, these results suggest that when participants encounter foil statements with high mutually exclusivity to extant true information, they are slower to respond than when foil statements have low mutually exclusivity to extant true information or are not related to extant mental information. The data suggest that individuals cannot withhold truth implications of foil statements with high mutually exclusivity information and must perform additional mental processing relative to foils with low mutually exclusivity information. Consistent with the 
FFABB evident in Study 1 and 2, the foil reaction time data of Study 1 and 2 indicate that naïve propositions are represented with a true stance, supporting the belief-default model view of ballistic propositional representation.

Trait judgment results were highly conserved across Studies 1 and 2 as both showed evidence of an "over-compensation effect" of false statements particularly in the moral trait assessments (Figure 3b, 4b). Thus, our participants (under "full" attention, i.e., without a condition of distraction or cognitive load) were effectively using the false cues in the stories to moderate trait judgments of the protagonists. This moderation even produced reliably higher trait assessments with stories where the false information was negative than with stories where the false information was positive. Thus, overall, healthy, younger- to middle-aged adults under the condition of full attention (Chen, 2002) did not show evidence that trait judgments were swayed by explicitly-labeled false information in the designed direction for each trait. However, on the individual analysis level, Study 2 provided evidence that individuals who tended to be swayed by the explicitly-labeled false information in the designed direction also commit more false as true (FasT) errors on the true/false recognition memory tests ${ }^{18}$ (see also Pantazi et al., 2018).

While Study 1 and 2's MPT data favor a belief-default perspective, other balanced, listbased false information research designs (Nadarevic \& Erdfelder, 2013, 2019) have reached opposing conclusions. In Study 3 we sought to extend our analysis by having participants undergo both a balanced, list-based false information paradigm (Gilbert et al., 1990; Pantazi et al., 2018) and an imbalanced, narrative-based false information paradigm (i.e., DJT). We predicted that participants would be more likely to rely on metacognitive guessing strategies

\footnotetext{
18 It should be noted that Study 1 did not find a significant correlation between FasT errors and critical trait judgments. This may be due to a lower number of participants in Study 1.
} 
(e.g., Batchelder \& Batchelder, 2008) during balanced, list-based false information paradigms which would artificially constrain rate differences between hits (TasT vs. FasF) and between veridical errors (TasF vs. FasT). Because MPT models only use response outcome proportions and cognitive processes assumptions to estimate parameter probabilities, MPT belief-related model conclusions are critically dependent on these response rate differences. Thus, in Study 3 we aimed to show that results from a balanced, list-based false information paradigm could support an MPT model with Cartesian assumptions, while DJT results from the same participants would support a belief-default MPT model.

Unfortunately, if this prediction is supported, it becomes difficult to adjudicate based only on behavioral outcomes whether the critical FasT errors are actual "believed" memories or are non-believed, "guessed" responses, in accordance with the increased true guessing bias hypothesis. However, if EDA indexes epistemic emotions during true/false recognition as predicted by the FTT, then there is a potential to independently assess whether FasT errors are "believed" (i.e., EDA of the FasT error corresponds to the EDA of TasT responses) or "guessed" (i.e., EDA of the FasT error does not correspond to the EDA of TasT responses). An establishment of reliable EDA differences between TasT, FasF, and NSasNS correct responses during both balanced, list-based and imbalanced, narrative-based true/false recognition memory tests would provide support to the FTT's contention of epistemic emotion elicitation during activation of a false tag and allow a novel method to identify whether FasT errors are "believed" or "guessed". The primary aim of Study 3 was to assess EDA during true/false recognition memory tests to evaluate these predictions.

Finally, to verify the "over-compensation" trait judgment effect found in the younger adults of Studies 1 and 2 and to extend our analyses to a sample of individuals poor at false 
information paradigms, we aimed to contrast older adults to younger adults in Study 3. Older adults under full attention tend to produce high rates of FasT errors relative to younger adults under full attention (Table 2) and are more influenced by false statements on trait judgments in the designed direction than younger adults (Chen, 2002; Chen \& Blanchard-Fields, 2000). This enables a direct comparison of age differences in FasT errors as well as FFABB.

\section{$\underline{\text { Study } 3}$}

To achieve these objectives we gave both younger and older adults the DJT and a listbased false information paradigm, the Hopi Language Task (Gilbert et al., 1990; Nadarevic \& Erdfelder, 2019), and recorded EDA during the tasks. In the HLT participants learn vocabulary terms from the "Hopi" language (but, in fact, they are nonsense terms) and then immediately receive feedback indicating whether the prior statement was true or false. For example, the proposition A monishna is a star is presented followed by either the signal word TRUE or FALSE. Following the learning phase, participants complete a subsequent true/false recognition memory test where they need to identify whether the statements were followed by the TRUE or FALSE signal word. The HLT is "balanced" in the sense that participants are exposed to equal numbers of true and false propositions during the learning phase. The primary aim of Study 3 was to examine EDA responses during the true/false recognition memory tests of the DJT and HLT. Secondary aims included comparing belief MPT models and contrasting developmental differences (older vs. younger adults) in the DJT and HLT.

\section{Method}

Overview. For participants who completed both the HLT and DJT, the HLT was always completed prior to the DJT. During the HLT participants were presented with novel propositions (Supplementary Materials) in a learning phase, and were explicitly signaled 2 seconds after 
whether the prior proposition was true or false. Subsequently, in a testing phase, participants were presented with the original propositions (in question form) and were asked to determine whether the proposition was signaled as true, false, or if it had never been presented (never seen). Several foils unrelated to true or false information from the learning phase were included in the true/false recognition memory test. Participants completed the DJT with the same procedures and stimuli as Studies 1 and 2. EDA was recorded throughout the cognitive testing via the thenar and hypothenar eminences of the participants' non-dominant hand.

Participants. A group of 57 individuals from a community-based sample participated in this study ${ }^{19}$. Thirty-two younger adults (YA; 16 male and 16 female, age range 30-50, age $M=$ 39.9 , age $S D=6.9$; years of education $M=16.3$, years of education $S D=1.8$ ) and 25 older adults (OA; 12 male and 13 female, age range 64-91, age $M=75.5$, age $S D=6.1$; years of education $M=16.2$, years of education $S D=2.8$ ) were administered the DJT. Of this sample, 9 older adults were not administered the HLT due to personal time constraints on cognitive testing. Thus, while all younger adults took both the DJT and HLT, only 16 older adults (7 male and 9 female, age range 64-91, age $M=76.2$, age $S D=6.7$; years of education $M=16.1$, years of education $S D=2.9$ ) were administered both the DJT and HLT. All participants were free from intellectual disability, learning disabilities, and dementia. Participants gave informed consent approved by the Institutional Review Board at the University of Iowa. This study was not preregistered prior to the research being conducted. No participants were excluded from this study post-hoc.

\footnotetext{
${ }^{19}$ The younger and older adults in this study were healthy comparison groups of a neuropsychological/neuropsychiatric project examining the neural correlates of belief and doubt processes. Thirty-eight patients with brain lesions and 26 patients with schizophrenia constituted the target groups which are the focus of separate studies. The data from these target groups were not analyzed at the time of this manuscript preparation.
} 
Stimuli and procedure. Prior to the informed consent process and to cognitive testing, each participant was queried regarding the amount of time toward cognitive testing they could provide. If the participant responded that they could only participate for 20-45 minutes, the experimenter administered only the DJT. If the participant responded that they could participant for more than 45 minutes, the experimenter first administered the HLT and then the DJT. Following the informed consent process, participants were escorted to a cognitive testing computer in a private room. Participants performed the HLT and DJT on standard Windowbased PC's running E-Prime software. The HLT paradigm mirrored Study 1 from Gilbert et al. (1990); see Supplementary Materials for complete procedure and stimuli. Electrodermal activity was recorded using the Biopac Systems MP150 and stored on a second PC for offline analysis. Two Ag- $\mathrm{AgCl}$ Kendall Meditrace 530 biopotential skin electrodes (Mansfield, MA) were affixed to the thenar and hypothenar eminences of the non-dominant palm. Continuous recording was initiated at the beginning of cognitive testing and was only terminated at the end of cognitive testing.

HLT learning phase. In the context of a language learning experiment, the computer presented 24 propositions, one at a time, in the form $A n X$ is a $Y$ where $X$ is an "Hopi Indian" noun and $Y$ its English equivalent. In fact, the ostensible Hopi noun was always a nonsense word. Two sessions of 12 propositions each were presented to the participants, with a true/false recognition memory test following each session. During the learning phase, each proposition was shown for 8 seconds. Two seconds after the proposition was presented, either the signal word TRUE or FALSE, was presented for 3 seconds. Ostensibly, this signal word indicated to the participant whether the previous proposition was accurate or inaccurate. Each session signaled 6 propositions as TRUE and 6 propositions as FALSE in a pseudorandomized order 
(Supplementary Materials). Participants were told that at the end of the session their knowledge of the Hopi vocabulary would be tested.

HLT true/false recognition memory test. During the testing phase, each of the propositions was rephrased in the form of a question (e.g., Is a monishna is a star?). Four foils were presented in the testing phase ( 2 in the first session and 2 in the second session). Thus, per session participants were presented 12 questions ( 5 true, 5 false, 2 never seen). Participants responded by pressing one of three buttons: true, false, or never seen. Overall, there were 24 critical trials where for 10 the correct answer was true, 10 the correct answer was false, and 4 the correct answer was never seen.

DJT stimuli and procedure. The procedure and stimuli of the DJT were identical to that of Study 1 .

Study 3 hypotheses. Hypothesis 1) In accordance with prior developmental research (e.g., Chen \& Blanchard-Fields, 2000), we hypothesized an increased FasT error rate in older adults relative to the FasT error rate in younger adults during the DJT. In addition, we predicted our MPT belief-default models will reflect this with a greater $d_{\text {false }}$ parameter estimate for younger adults than for older adults. Hypothesis 2) During the DJT, older adults will be more influenced by the false statements on trait judgments in the designed direction relative to younger adults. Hypothesis 3) Participants (both younger and older adults) will not show increased FasT errors relative to TasF errors on the HLT. We predict our Cartesian assumption MPT models will reflect this by providing a better fit to the HLT data than our MPT belief-default model (Figure 5). Hypothesis 4) During both DJT and HLT true/false recognition memory tests, hits (TasT and FasF) will correlate with increased EDA magnitude responses relative to EDA magnitude responses of correct rejections (NSasNS). Moreover, the type of hit (TasT or FasF) will predict 
EDA magnitude differences. Hypothesis 5) If hypothesis 4 is supported, veridical errors may be retroactively binned into "believed" and "guessed" categories based on EDA magnitude. Given this method, we hypothesize that participants (both older and younger adults) will produce increased "believed" FasT errors relative to "believed" TasF errors on both the DJT and HLT. Hypothesis 6) Finally, we hypothesized older adults will have increased "believed" FasT errors relative to younger adults.

Initial statistical analyses. DJT statistical analyses closely corresponded with Study 1 with the exception of an additional group-level fixed factor (younger and older adults) in our mixed-effects models. Equivalent statistical analyses were conducted on the HLT data. All twoand three-way interactions of fixed factors were examined in each model. Reaction time statistical model details and results for Study 3 are provided in the Supplementary Materials.

EDA analyses proceeded by converting participants' tonic waves into phasic waves using the Biopac Systems AcqKnowledge 4 software package (Goleta, CA). Specifically, the tonic EDA wave was resampled at 62.5 samples/second with a linear interpolation method and a fixed linear phase low pass $1.0 \mathrm{~Hz}$ filter was applied to the tonic wave. The tonic wave was then converted into a phasic wave using a 5 second baseline estimation widow width. Of interest to our hypotheses were EDA magnitudes during the DJT and HLT true/false recognition memory tests. A 4-second latency window was specified around the participants' responses to each item in the recognition memory tests, which allowed for the possibility of a minimal delay between a decision and a behavioral response (Dawson et al., 2000). The window's parameters were subjected to some variation by a blind EDA analyst ${ }^{20}$ due to differences in each participant's speed to complete items in the recognition memory tests. Within each window the peak

\footnotetext{
${ }^{20} \mathrm{EDA}$ analysts did not know the participants' responses to the recognition memory tests.
} 
magnitude of the phasic EDA wave was recorded. No minimum skin conductive response (SCR) cutoff was determined because of the observation of reliable differences in magnitudes under $.02 \mu \mathrm{S}$ (answering recognition memory tests may not be sufficiently arousing to reach traditional thresholds of SCR, see also Morris et al., 2008). At these small values, individual differences in EDA magnitudes produced by extraneous factors, such as skin thickness, hydration, and eccrine sweat gland functioning, are exaggerated (Dawson et al., 2000). Thus, EDA magnitudes within-subject were converted to standard scores (zEDA) to adjust for individual differences and allow for comparison across participants (Ben-Shakhar, 1985). For the DJT, zEDA data were produced both within an individual and a story; i.e., zEDAs were calculated using each participant's EDA story (e.g., Mike's) mean and standard deviation. For the HLT, zEDA data were produced within an individual only; i.e., zEDAs were calculated using each participant's EDA mean and standard deviation across both sessions. In accordance with the judgment and reaction time analyses, zEDA outliers were excluded based on the median absolute deviation with a constant of 3 (Leys et al., 2013). See Supplementary Materials for HLT EDA statistical approaches and data analyses.

DJT old statements statistics. For the old statements mixed-effects model, there were three fixed factors: classification (hit, veridical error, miss); statements (true, false); and group $(Y A, O A)$. Intercepts and slopes for participants and recognition memory test items were added as random effects. We examined hypothesis 1 with the inclusion of a classification by statements by group interaction.

$\underline{\text { DJT foil statements statistics. }}$. There were three fixed factors in our foil statements mixed-effects model: answer (correct rejection, "true" false alarm, "false" false alarm); 
statements $\left(N S^{\emptyset}, N S^{\sim t}, N S^{\neq t}, N S^{\sim f}\right)$; and group $(Y A, O A)$. Intercepts and slopes for participants and recognition memory test items were added as random effects.

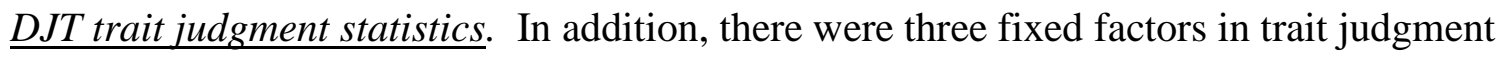
mixed-effects model: false valence (positive falses, negative falses); critical trait (cognitive assessment, moral assessment, likeable assessment); and group (YA,OA). Intercepts for participants were added as random effects. Hypothesis 2 was examined with a false valence by group interaction.

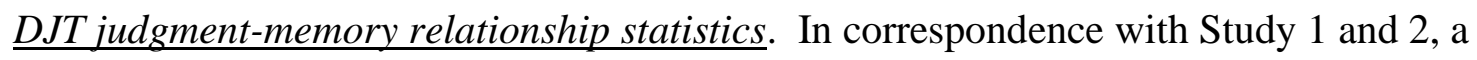
Pearson correlation between trait judgments and FasT error rates was conducted with the whole sample (Supplementary Materials).

DJT EDA peak magnitude statistics. Prior to the conversation of EDA magnitudes to standard scores (zEDA), average EDA peak magnitudes were calculated across all participants for each type of recognition memory test behavioral response: true, false, and never seen.

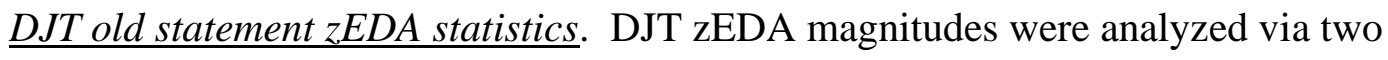
mixed-effects models to allow for both a direct comparison of the type of response (hits/veridical errors/misses) but also of the answer provided (truelfalse/never seen). Examination of DJT old statements zEDA included fixed factors classification (hit, veridical error, miss); statements (true, false); and group (YA,OA); which allowed a classification by statements interaction addressing hypothesis 4 . Intercepts for participants and recognition memory test items were added as random effects.

$\underline{\text { DJT all statement zEDA statistics. }}$ To examine zEDA to the answer provided, we collapsed the differing foil conditions and outcomes together and included old statements for an 
overall analysis $^{21}$. Thus, our all statement zEDA mixed-effects model included fixed factors answer (true, false, never seen); statements (true, false, foil); and group (YA,OA); which allowed an answer main effect and an answer by statements interaction further addressing hypothesis 4 . Again, intercepts for participants and recognition memory test items were added as random effects.

$\underline{H L T \text { old statements statistics. }}$. Two binomial mixed-effects models using the logit link target option in SPSS were conducted on the HLT true/false recognition memory test result data to examine a direct comparison of the type of response (hits/veridical errors/misses) as well as the answer provided (truelfalse/never seen). No outliers were excluded from the recognition memory test results. For the old statements mixed-effects model, the fixed factors included classification (hit, veridical error, miss); statements (true, false); and group (YA,OA). Thus, as with the analysis of DJT old statements, the target variable was repeated three times for each of the 20 old items participants answered during the HLT recognition memory tests. We examined hypothesis 3 with the inclusion of a classification by statements interaction. Intercepts and slopes for participants and recognition memory test items were added as random effects.

$\underline{\text { HLT all statements statistics. }}$ Similarly, a binomial all statement mixed-effects model was conducted which included the fixed factors answer (true, false, never seen); statements (true, false, foil); and group (YA,OA). The target variable was repeated three times for each of the 24 items participants answered during the HLT recognition memory tests. We further examined hypothesis 3 with the inclusion of an answer by statements interaction. Intercepts and slopes for participants and recognition memory test items were added as random effects.

\footnotetext{
${ }^{21}$ See Supplementary Materials for mixed-effects model with a non-collapsed foil conditions (i.e., with a statements fixed factor including true, false, $N S^{\varnothing}, N S^{\sim t}, N S^{\star t}$, and $N S^{\sim f}$ ).
} 
DJT and HLT multinomial processing tree statistics. Study 3 contrasted the same DJT MPT models as Study 1 (Figure 2). However, two unique MPT models were designed to assess Cartesian and belief-default assumptions from HLT outcomes (Figure 5). Similar to the DJT MPT model with belief-default assumption, the HLT MPT model with belief-default assumptions includes a $d_{\text {false }}$ parameter that separates correctly recognized propositions into FasF and FasT responses (Figure 2b, 5b). Moreover, the HLT MPT model with Cartesian assumptions is comparable to the DJT MPT model with Cartesian assumption as it includes a $d_{\text {true }}$ parameter (Figure 2a, 5a). We again echoed Nadarevic and Erdfelders' (2013) constraints of recognition parameter equality $\left(D_{\text {true }}=D_{\text {false }}\right)$ and guessing true parameter equality $\left(a_{\text {true }}=g_{\text {true }}\right.$ $=.50$ ) for the HLT MPT model with Cartesian assumptions. Parameters $d_{\text {true }}$ and $d_{\text {false }}$ were free to vary as no constraints were placed on them. In addition, both the HLT MPT model with Cartesian assumptions and the HLT MPT model with belief-default assumptions include a onehigh threshold assumption which is necessary for model fit with the low numbers of foils (Figure 5) relative to true and false items.

Post-hoc statistical analyses. Following the initial zEDA analyses and interpretation of these results, we conducted two post-hoc binomial mixed-effects models on the old statements of the DJT and HLT. Given the ordinal nature of the zEDA results corresponding to true, false, and never seen answering (see Study 3 Results); we sought to define each recognition memory test outcome based on zEDA elicited. Namely, if EDA is measuring epistemic emotion elicitation following a successful memory search, then guessed responses should not show this epistemic emotion elicitation. Thus, the zEDA of guessed responses should be comparable to the zEDA of correctly rejected foils (i.e., NSasNS). Based on this logic, hits (TasT, FasF) and veridical errors (TasF, FasT) were binned into either "believed" or "guessed" categories based on the 
zEDA elicited during the response. Our bins were established via the midpoints between the ordinal zEDA TasT, FasF, and NsasNS response means (see Supplementary Materials for HLT zEDA response means). Thus, in the DJT, a true response was categorized as "believed" if the zEDA was greater than .25 and a false response was categorized as "believed" if the zEDA was between -.39 and .25 . True and false responses were categorized as "guessed" if the zEDA was below -.39. Never seen responses were categorized as low zEDA if the zEDA was below -.39 and high zEDA if the zEDA was above -.39. In the HLT, a true response was categorized as "believed" if the zEDA was greater than .05 and a false response was categorized as "believed" if the zEDA was between -.46 and .05. True and false responses were categorized as "guessed" if the zEDA was below -.46. Never seen responses were categorized as low zEDA if the zEDA was below -.46 and high zEDA if the zEDA was above -.46. See Supplementary Materials for HLT zEDA believed vs. guessed statistical approaches and data analyses.

DJT old statements zEDA believed vs. guessed statistics. A follow-up binomial mixedeffects model using the logit link target option in SPSS was conducted on the DJT true/false recognition memory data. No outliers were excluded from the recognition memory test results. The fixed factors included zEDA classification (hit "believed", hit "guessed", veridical error "believed”, veridical error "guessed”, miss “low zEDA”, miss "high zEDA”); statements (true, false); and group $(Y A, O A)$. Thus, the target variable was repeated six times for each of the 48 items participants answered during the recognition memory tests. We examined hypothesis 5 with the inclusion of a $z E D A$ classification by statements interaction and hypothesis 6 with a three-way interaction. Intercepts and slopes for participants and recognition memory test items were added as random effects.

Results 
DJT old statements results. The DJT results from Study 3 replicated many of the basic findings of Studies 1 and 2. There was a main effect of classification, $F(2,8196)=1339.38, p<$ .001. Old statements were more likely to be hits than veridical errors, $t(8196)=72.64, p<.001$, $d=1.99,95 \%$ CI $[1.92,2.05]$, and misses, $t(8196)=77.16, p<.001, d=2.14,95 \%$ CI [2.08, 2.22]. No significant differences were observed between misses and veridical errors. There was also a main effect of statements, $F(1,8196)=5.98, p=.015$, such that false statements were greater than true statements in the model, $t(8196)=2.48, p=.013, d<0.01,95 \%$ CI $[0, .04]$. There was also a classification by statements interaction, $F(2,8196)=118.92, p<.001$. True statements were more correctly classified as true (TasT) than false statements correctly classified as false $(\mathrm{FasF}), t(8196)=11.91, p<.001, d=.49,95 \% \mathrm{CI}[.41, .57]$. Corresponding to Studies 1 and 2, false statements were more likely misclassified as true (FasT) than true statements were misclassified was false (TasF), $t(8196)=11.24, p<.001, d=.56,95 \%$ CI $[.49, .64]$ (Figure 6a). While there was no significant main effect of group, there was a two-way interaction between classification and group, $F(2,8196)=47.65, p<.001$. Younger adults were more likely to correctly answer with a hit than older adults, $t(8196)=7.33, p<.001, d=.38,95 \%$ CI $[.31, .46]$. Older adults were more likely to incorrectly respond with a veridical error, $t(8196)=5.12, p<$ $.001, d=.38,95 \%$ CI $[.31, .46]$, and a miss, $t(8196)=3.09, p=.002, d=.15,95 \%$ CI $[.07, .22]$, than younger adults. Finally, there was a three-way interaction observed between classification, statements, and group, $F(2,8196)=17.91, p<.001$. Younger adults were more likely to correctly classify false statements as false (FasF) relative to older adults, $t(8196)=10.85, p<$ $.001, d=.61,95 \% \mathrm{CI}[.50, .72]$. In support of hypothesis 1 , older adults were more likely to incorrectly classify false statements as true (FasT) relative to younger adults, $t(8196)=9.88, p<$ $.001, d=.57,95 \% \mathrm{CI}[.46, .68]$ (Figure 6a). Older adults were also more likely to incorrectly 
classify false statements as never seen (FasNS) relative to younger adults, $t(8196)=3.10, p=$ $.002, d=.20,95 \%$ CI $[.09, .31]$. Critically, no significant differences were observed between the groups regarding TasT, TasF, nor TasNS outcomes (Figure 6a).

DJT foil statements results. The DJT foil statements mixed-effects model found a main effect of answer, $F(2,8184)=1288.13, p<.001$. Foil statements were more correctly identified as never seen (NSasNS) than misclassified as true (NSasT), $t(8184)=85.49, p<.001, d=2.45$, $95 \%$ CI $[2.38,2.52]$ or misclassified as false (NSasF), $t(8184)=65.78, p<.001, d=1.75,95 \%$ CI $[1.69,1.82]$. Foil statements were more likely to be misclassified as false (NSasF) than misclassified as true (NSasT), $t(8184)=8.65, p<.001, d=.37,95 \%$ CI $[.32, .43]$. There was also an answer by statements interaction, $F(6,8184)=48.35, p<.001$. Foil statements not related to true nor false statements were more correctly rejected (NS ${ }^{\left.\emptyset_{a s N S}\right)}$ than foil statements similar but not mutually exclusive to true statements $\left(\mathrm{NS}^{-\mathrm{t}}\right.$ asNS), $t(8184)=2.50, p=.012, d=$ $.16,95 \% \mathrm{CI}[.05, .28]$, foil statements similar and mutually exclusive to true statements $\left(\mathrm{NS}^{\ddagger \mathrm{t}}\right.$ asNS $), t(8184)=12.70, p<.001, d=.66,95 \%$ CI $[.55, .76]$, or foil statements similar to false statements ( $\left.\mathrm{NS}^{\sim \mathrm{f}} \mathrm{aSNS}\right), t(8184)=7.78, p<.001, d=.44,95 \% \mathrm{CI}[.33, .55] . \mathrm{NS}^{\sim \mathrm{t}}$ asNS responses were more likely than $\mathrm{NS}^{\neq t}$ asNS responses, $t(8184)=9.57, p<.001, d=.49,95 \% \mathrm{CI}$ $[.39, .60]$, and $\mathrm{NS}^{\sim f}$ asNS responses, $t(8184)=5.01, p<.001, d=.28,95 \% \mathrm{CI}[.17, .39]$. $\mathrm{NS}^{\sim \mathrm{f}}$ asNS responses were more likely than $\mathrm{NS}^{\ddagger t}$ asNS responses, $t(8184)=4.36, p<.001, d=$ $.22,95 \% \mathrm{CI}[.12, .33]$. We did observe that foil statements not related to true nor false statements were less likely classified as true (NS $\left.{ }^{\emptyset_{a s T}}\right)$ than foil statements similar and mutually exclusive to true statements $\left(\mathrm{NS}^{\ddagger \mathrm{t}} \mathrm{asT}\right), t(8184)=2.45, p=.014, d=.22,95 \% \mathrm{CI}[.12, .32]$ and foil statements similar to false statements $\left(\mathrm{NS}^{\sim f}\right.$ asT), $t(8184)=2.12, p=.034, d=.21,95 \% \mathrm{CI}$ $[.10, .32]$. Corresponding to Studies 1 and 2 , foil statements similar and mutually exclusive to 
true statements were more likely classified as false $\left(\mathrm{NS}^{\neq \mathrm{t}} \mathrm{asF}\right)$ than foil statements not related to

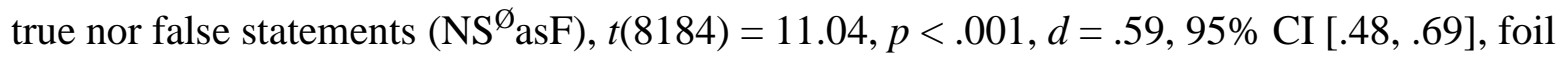
statements similar but not mutually exclusive to true statements $\left(\mathrm{NS}^{\sim \mathrm{t}} \mathrm{asF}\right), t(8184)=9.92, p<$ $.001, d=. .52,95 \% \mathrm{CI}[.41, .63]$, and foil statements similar to false statements ( $\left.\mathrm{NS}^{\sim \mathrm{f}} \mathrm{asF}\right)$, $t(8184)=4.45, p<.001, d=.23,95 \% \mathrm{CI}[.12, .33]$ (Figure 6a). $\mathrm{NS}^{\sim \mathrm{f}}$ asF errors were more likely than $\mathrm{NS}^{\emptyset_{\text {asF }}}$ errors, $t(8184)=6.24, p<.001, d=.38,95 \% \mathrm{CI}[.27, .48]$, and $\mathrm{NS}^{\sim \mathrm{t}}$ asF errors, $t(8184)=5.29, p<.001, d=.31,95 \%$ CI $[.20, .43]$. While there was no significant main effect of group, there was a two-way interaction between answer and group, $F(2,8184)=16.13, p<$ .001. Younger adults were more likely to correctly answer with a never seen response (NSasNS) than older adults, $t(8184)=3.63, p<.001, d=.15,95 \%$ CI $[.08, .23]$. Older adults were more likely to incorrectly respond with a false response (NSasF) than younger adults, $t(8184)=4.36, p$ $<.001, d=.18,95 \% \mathrm{CI}[.11, .26]$. Interestingly, there was not a significant three-way interaction between classification, statements, and group which indicates that the FFABB is not as strongly predicted from age as the FasT error.

DJT trait judgment results. Of the total responses $2.05 \%$ were excluded as outliers. Similar to Studies 1 and 2, there was a main effect of critical trait, $F(2,603.73)=10.38, p<$ .001. Cognitive assessments (intelligent/clever) had reduced ratings relative to likeable assessments, $M D=-.64, p<.001, d=.42,95 \% \mathrm{CI}[.23, .61]$. In addition, there was a false valence by critical trait interaction, $F(2,603.24)=4.82, p=.008$. Positive falses had increased ratings relative to negative falses in cognitive assessments specifically, $M D=.57, p=.004, d=$ $.37,95 \%$ CI $[.11, .64]$. While there was no main effect of group, there was a false valence by group interaction, $F(2,603.84)=18.41, p<.001$. In support of hypothesis 2 , older adults had decreased ratings to negative falses relative to positive falses, $M D=-.71, p<.001, d=.34,95 \%$ 
CI $[.12, .57]$, whereas younger adults did not show a significant difference between negative falses and positive falses (Figure 6b). Older adults did not demonstrate an "over-compensation effect" prevalent in the trait judgment results of Studies 1 and 2. Thus, only older adults were swayed by the valence of the false statements in the designed direction (i.e., increased trait ratings for positive falses and decreased trait ratings for negative falses).

DJT judgment-memory relationship results. In correspondence with Study 2, we found a strong positive correlation between FasT errors and impact of the explicitly-labeled false statements on participant critical trait judgments, $r(57)=.68, p<.001$.

DJT EDA peak magnitude results. Across all participants in the DJT recognition memory test, EDA peak magnitudes corresponded to the answer provided: true answering ( $M=.084 \mu \mathrm{S}$; $S D=.043 \mu \mathrm{S})$, false answering $(M=.052 \mu \mathrm{S} ; S D=.035 \mu \mathrm{S})$, and never seen answering $(M=$ $.040 \mu \mathrm{S} ; S D=.033 \mu \mathrm{S})$.

DJT old statement zEDA results. Of the total responses $2.56 \%$ were excluded as outliers. The DJT old statement zEDA mixed-effects model showed a main effect of classification, $F(2$, $2633.05)=87.83, p<.001$. Misses $($ TasNS, FasNS) had reduced zEDA relative to hits $($ TasT, FasF), $M D=-.82, p<.001, d=.95,95 \%$ CI $[.81,1.10]$, and veridical errors (TasF, FasT), $M D=$ $-.90, p<.001, d=1.50,95 \%$ CI $[1.30,1.71]$. No significant differences were observed between hits and veridical errors. There was also a classification by statements interaction, $F(2,2619.39)$ $=67.61, p<.001$. In support of hypothesis 4 , TasT responses had greater zEDA magnitudes than FasF responses, $M D=.97, p<.001, d=1.07,95 \%$ CI $[.98,1.16]$ (Figure 6c). In addition, FasT errors had greater zEDA magnitudes than TasF errors, $M D=.51, p=.003, d=.58,95 \% \mathrm{CI}$ $[.19, .97]$. Moreover, zEDA was predicted by the true/false recognition memory test answer. TasT responses had greater zEDA magnitudes than TasF errors, $M D=.67, p<.001, d=.65$, 
95\% CI [.28, 1.02], and TasNS errors, $M D=1.33, p<.001, d=1.34,95 \%$ CI $[1.12,1.56]$. TasF errors had greater zEDA magnitudes than TasNS errors, $M D=.67, p=.001, d=1.51,95 \% \mathrm{CI}$ $[1.05,1.98]$. FasT errors had greater zEDA magnitudes than FasF responses, $M D=.82, p<$ $.001, d=1.16,95 \% \mathrm{CI}[1.01,1.30]$, and FasNS errors, $M D=1.13, p<.001, d=1.41,95 \% \mathrm{CI}$ $[1.17,1.66]$. FasF responses had greater zEDA magnitudes than FasNS errors, $M D=.31, p=$ $.001, d=.60,95 \% \mathrm{CI}[.40, .80]$ (Figure $6 \mathrm{c}$ ). Of note there was no significant main effect of group nor of any two- or three-way interaction with group.

DJT all statement zEDA results. Given our initial results that suggested zEDA was predicted by the true/false recognition test answer, we collapsed the differing foil conditions together and included old statements for an overall DJT zEDA mixed-effects model (see Supplementary Materials for a non-collapsed foil zEDA mixed-effect model). Of the total responses $4.72 \%$ were excluded as outliers. The DJT all statement zEDA mixed-effects model showed a main effect of answer, $F(2,5176.82)=323.67, p<.001$. In accordance with hypothesis 4, true responding (TasT, FasT, NSasT) had greater zEDA magnitudes than false responding (TasF, FasF, NSasF), MD $=.72, p<.001, d=1.03,95 \%$ CI $[.95,1.07]$, and never seen responding (TasNS, FasNS, NSasNS), $M D=1.15, p<.001, d=1.61,95 \%$ CI $[1.54,1.68]$. Moreover, false responding (TasF, FasF, NSasF) had greater zEDA magnitudes than never seen responding (TasNS, FasNS, NSasNS), MD =.43, $p<.001, d=.64,95 \%$ CI $[.55, .71]$ (Figure 6c). Thus, zEDA magnitudes during true/false recognition memory tests corresponded to the answer provided in an ordinal nature: true responses with the highest magnitude zEDA, false responses with middle magnitude zEDA, and never seen responses with the lowest magnitude zEDA. There was also an answer by statements interaction, $F(4,5153.40)=2.67, p=.030$. TasT responses had greater zEDA magnitudes than TasF errors, $M D=.74, p<.001, d=.65$, 
95\% CI [.28, 1.02], and TasNS errors, $M D=1.34, p<.001, d=1.34,95 \%$ CI $[1.12,1.56]$. TasF errors had greater zEDA magnitudes than TasNS errors, $M D=.61, p<.001, d=1.51,95 \% \mathrm{CI}$ $[1.05,1.98]$. FasT errors had greater zEDA magnitudes than FasF responses, $M D=.78, p<$ $.001, d=1.16,95 \% \mathrm{CI}[1.01,1.30]$, and FasNS errors, $M D=1.12, p<.001, d=1.41,95 \% \mathrm{CI}$ $[1.17,1.66]$. In addition, FasF responses had greater zEDA magnitudes than FasNS errors, $M D$ $=.34, p<.001, d=.60,95 \% \mathrm{CI}[.40, .80]$. NSasT errors had greater zEDA magnitudes than NSasF errors, $M D=.64, p<.001, d=.82,95 \%$ CI $[.60,1.04]$, and NSasNS responses, $M D=$ $.99, p<.001, d=1.74,95 \%$ CI $[1.54,1.94]$. NSasF errors had greater zEDA magnitudes than NSasNS responses, $M D=.34, p<.001, d=.61,95 \%$ CI $[.50, .72]$ (Figure 6c). Finally, TasT responses had greater zEDA magnitudes than FasT errors, $M D=.17, p=.028, d=.18,95 \% \mathrm{CI}$ $[.04, .31]$, and NSasT errors, $M D=.31, p<.001, d=.31,95 \%$ CI $[.12, .51]$ (Figure 6c). Again, there was no significant main effect of group nor of any two- or three-way interaction with group.

HLT old statements results. For the old statements mixed-effects of a balanced, listedbased false information paradigm (i.e., the HLT), we found a main effect of classification, $F(2$, $2868)=271.50, p<.001$. Old statements were more likely to be hits than veridical errors, $t(2868)=17.33, p<.001, d=.89,95 \%$ CI $[.80, .99]$, and misses, $t(2868)=35.20, p<.001, d=$ $1.82,95 \%$ CI $[1.72,1.93]$. In addition, old statements were more likely to be veridical errors than misses, $t(2868)=13.87, p<.001, d=.70,95 \%$ CI $[.60, .79]$ (Figure 6d). In accordance with hypothesis 3 and in opposition to the DJT results, we did not observe a significant classification by statements interaction indicating that FasT errors were increased relative to TasF errors (Figure 6d). However, there was a two-way interaction between classification and group, $F(2,2868)=5.89, p=.003$. Younger adults were more likely to correctly answer with a 
hit than older adults, $t(2868)=2.34, p=.019, d=.16,95 \%$ CI $[.02, .31]$, and older adults were more likely to incorrectly respond with a veridical error, $t(2868)=2.43, p=.015, d=.17,95 \%$ CI $[.04, .31]$ (Figure 6d). Of note our model did not reveal a main effect of group nor a threeway interaction between classification, statements, and group.

HLT all statements results. A mixed-effects model for all HLT statements found a significant answer by statements interaction, $F(4,3438)=156.79, p<.001$. True statements were more correctly classified as true (TasT) than true statements incorrectly classified as false $($ TasF $), t(3438)=12.90, p<.001, d=.92,95 \%$ CI $[.79,1.05]$, and true statements incorrectly classified as never seen (TasNS), $t(3438)=25.70, p<.001, d=1.86,95 \%$ CI $[1.71,2.02]$. TasF errors were increased relative to TasNS errors, $t(3438)=9.75, p<.001, d=.70,95 \%$ CI $[.57$, .83]. False statements were more correctly classified as false (FasF) than false statements incorrectly classified as true $($ FasT $), t(3438)=11.62, p<.001, d=.87,95 \%$ CI $[.73,1.00]$, and false statements incorrectly classified as never seen (FasNS), $t(3438)=24.12, p<.001, d=1.78$, 95\% CI $[1.64,1.95]$. FasT errors were increased relative to FasNS errors, $, t(3438)=9.86, p<$ $.001, d=.70,95 \%$ CI $[.57, .83]$. Finally, foil statements were more correctly classified as never seen (NSasNS) than foil statements incorrectly classified as true (NSasT), $t(3438)=39.16, p<$ $.001, d=5.39 ., 95 \%$ CI $[4.96,5.82]$, and foil statements incorrectly classified as false (NSasF), $t(3438)=36.81, p<.001, d=4.74,95 \%$ CI $[4.35,5.14]$ (Figure 6d). In addition, there was a significant three-way interaction between classification, statements, and group, $F(4,3438)=$ $3.26, p=.011$. Younger adults were more likely to correctly answer false statements as false $($ FasF) than older adults, $t(3438)=2.36, p=.018, d=.23,95 \%$ CI $[.04, .43]$, and older adults were more likely to incorrectly answer false statements as true (FasT) than younger adults, $t(3438)=2.09, p=.036, d=.21,95 \%$ CI $[.02, .40]$. 
DJT and HLT multinomial processing tree results. In accordance with Studies 1 and 2, the DJT MPT model with Cartesian assumptions poorly fit both the younger adult data ${ }^{22}, \mathrm{G}^{2}(1)=$ 87.95, $p<.001$, and the older adult data, $\mathrm{G}^{2}(1)=114.05, p<.001$. Replicating Studies 1 and 2, the DJT MPT model with belief-default assumptions fit both the younger adult data, $\mathrm{G}^{2}(1)=.14$, $p=.710$, and the older adult data, $\mathrm{G}^{2}(1)=.51, p=.475$, well. Again, the parameter estimates for the DJT MPT model with belief-default assumptions corresponded to the main effects reported in the old and foil statements mixed-effects models (Table 3). Constraining $D$ to be equal for true, false and foil statements led to a good model fit in the younger adult data, $\Delta \mathrm{G}(2)=1.08, p=$ .583 , and a moderate model fit in the older adult data, $\Delta \mathrm{G}(2)=6.00, p=.050$. $D_{\text {true }}$ for older adults was significantly reduced relative to $D_{\text {true }}$ for younger adults, $\Delta \mathrm{G}(1)=6.28, p=.012$; and $D_{\text {false }}$ for older adults was also significantly reduced relative to $D_{\text {false }}$ for younger adults, $\Delta \mathrm{G}(1)=$ 8.81, $p=.003$. $D_{N S}$ did not significantly differ between younger and older adults, $\Delta \mathrm{G}(1)=.21, p$ $=.648$. Importantly, $d_{\text {false }}$ in the younger adult data corresponded with Studies 1 and 2 as it was estimated at .91. However, the older adults $d_{\text {false }}$ parameter was estimated at .66 (Table 3). Remarkably, of the false statements recognized, where younger adults mistakenly identified $9 \%$ as true (1- $\left.d_{\text {false }}\right)$, older adults mistakenly identified $34 \%$ as true. In support of hypothesis $1, d_{\text {false }}$ for older adults was significantly reduced relative to $d_{\text {false }}$ for younger adults, $\Delta \mathrm{G}(1)=40.05, p<$ .001. The "false" false alarm parameter for foil statements with high mutual exclusivity to true information $\left(z_{\neq t}\right)$ was greater relative to "false" false alarm parameter for foil statements with low mutual exclusivity to true information $\left(z \emptyset, z_{\sim t}\right)$ in both younger adults, $\Delta \mathrm{G}(1)=104.80, p<.001$, and older adults, $\Delta \mathrm{G}(1)=74.57, p<.001$. In accordance with Study $1, z_{\neq t}$ was greater relative to the "false" false alarm parameter for foil statements that were similar to false information $(z \sim f)$ in

\footnotetext{
${ }^{22}$ See Supplementary Materials for the Study 3's DJT data applied to the "hybrid" MPT model.
} 
both younger adults, $\Delta \mathrm{G}(1)=6.71, p=.010$, and older adults, $\Delta \mathrm{G}(1)=13.22, p<.001$.

Parameter $z_{\sim f}$ was also greater than $z \varnothing$ and $z_{\sim t}$ in both younger adults, $\Delta \mathrm{G}(1)=45.89, p<.001$, and older adults, $\Delta \mathrm{G}(1)=12.42, p<.001$. Parameter $z_{\neq t}$ in older adults was greater relative to $z_{\neq t}$ in younger adults, $\Delta \mathrm{G}(1)=8.48, p=.004$. Parameters $z \emptyset, z \sim t$ were also increased in older adults relative to $z \emptyset, z_{\sim t}$ in younger adults, $\Delta \mathrm{G}(1)=6.78, p=.009$. There was not a significant difference between younger and older adults for parameter $z_{\sim f}, \Delta \mathrm{G}(1)=1.22, p=.270$. Similar to Studies 1 and 2, constraining $s_{\text {true }}$ and $s_{f a l s e}$ to be equal led to a good model fit in both younger adults, $\Delta \mathrm{G}(1)=3.20, p=.074$, and older adults, $\Delta \mathrm{G}(1)=.32, p=.575$. Finally, there was not a significant difference between younger and older adults for parameter $s_{\text {true }}, \Delta \mathrm{G}(1)=2.81, p=$ .094, sfalse, $\Delta \mathrm{G}(1)=.16, p=.670, b, \Delta \mathrm{G}(1)=.53, p=.465$, and $n, \Delta \mathrm{G}(1)=.15, p=.694$.

However, in contrast, MPT analyses conducted on the balanced, list-based false information paradigm data (i.e., the HLT) supported our MPT model with Cartesian assumptions rather than the MPT model with belief-default assumptions. The HLT MPT model with beliefdefault assumptions (Figure 5a) poorly fit both the younger adult data, $\mathrm{G}^{2}(2)=232.68, p<.001$, and the older adult data, $\mathrm{G}^{2}(2)=125.51, p<.001$. In support of hypothesis 3 , the HLT MPT model with Cartesian assumptions fit both the younger adult data, $\mathrm{G}^{2}(2)=.37, p=.829$, and the older adult data, $\mathrm{G}^{2}(2)=2.46, p=.293$, well. Parameter estimates for the HLT MPT model with Cartesian assumptions corresponded to the main effect reported in the HLT mixed-effects models (Table 4). Parameters $d_{\text {true }}$ and $d_{\text {false }}$ were not significantly different in either the younger adult data, $\Delta \mathrm{G}(1)=.02, p=.889$, or older adult data, $, \Delta \mathrm{G}(1)=.59, p=.444$. Parameters $D_{\text {true }}$ and $D_{\text {false }}$ did not significantly differ between younger and older adults, $\Delta \mathrm{G}(1)=.01, p=.944$. In addition, parameter $b$ did not significantly differ between younger and older adults, $\Delta \mathrm{G}(1)=.50$, $p=.478$. While $d_{\text {true }}$ also did not significantly differ between younger and older adults, $\Delta \mathrm{G}(1)=$ 
$1.61, p=.205 ; d_{\text {false }}$ was greater in younger adult relative to older adults, $\Delta \mathrm{G}(1)=5.10, p=.024$. This data provides evidence that even in a balanced, list-based false information paradigm where a participant may more easily use base-rate information, older adults are preferentially poor at correctly identifying recognized false information as false.

Post-hoc DJT old statements zEDA believed vs. guessed results. A follow-up binomial mixed-effects model using zEDA to delineate "believed" versus "guessed" DJT true/false recognition memory data found a main effect of $z E D A$ classification, $F(5,16392)=341.31, p<$ .001. Old statements were more likely to be hit "believed" (i.e., TasT and FasF) than hit "guessed", $t(16392)=16.28, p<.001, d=.45,95 \%$ CI $[.40, .51]$, veridical error "believed" (i.e., TasF and FasT), $t(16392)=30.13, p<.001, d=.91,95 \%$ CI $[.86, .97]$, veridical error "guessed", $t(16392)=34.19, p<.001, d=1.12,95 \%$ CI [1.06, 1.18], miss "low $z E D A$ " (i.e., TasNS and FasNS), $t(16392)=30.72, p<.001, d=.95,95 \%$ CI $[.90,1.01]$, and miss "high $z E D A ", t(16392)=33.45, p<.001, d=1.08,95 \% \mathrm{CI}[1.02,1.13]$. Old statements were more likely to be hit "guessed" than veridical error "believed", $t(16392)=13.80, p<.001, d=.44$, $95 \%$ CI $[.40, .50]$, veridical error "guessed", $t(16392)=17.82, p<.001, d=.65,95 \%$ CI [.60, $.71]$, miss "low zEDA", $t(16392)=14.17, p<.001, d=.48,95 \%$ CI $[.43, .54]$, and miss "high $z E D A ", t(16392)=17.02, p<.001, d=.61,95 \%$ CI $[.55, .66]$. Old statements were also more likely to be veridical error "believed" than veridical error "guessed", $t(16392)=4.00, p<.001$, $d=.24,95 \%$ CI $[.19, .30]$, and miss "high zEDA", $t(16392)=3.07, p=.002, d=.19,95 \% \mathrm{CI}$ $[.14, .24]$. Moreover, old statements were less likely to be veridical error "guessed" than miss "low $z E D A ", t(16392)=4.35, p<.001, d=.20,95 \% \mathrm{CI}[.15, .26]$. Finally, old statements were more likely to be miss "low zEDA" than miss "high zEDA", $t(16392)=3.34, p=.001, d=.15$, $95 \%$ CI $[.09, .20]$. There was also a main effect of statements, $F(1,16392)=14.97, p<.001$, 
such that false statements were greater than true statements in the model, $t(16392)=3.92, p<$ $.001, d=.01,95 \% \mathrm{CI}[0, .04]$. A main effect of group was observed, $F(1,16392)=5.59, p=$ .018 , such that $O A$ was greater than $Y A$ in the model, $t(16392)=2.35, p=.019, d=.02,95 \% \mathrm{CI}$ $[.01, .05]$. There was a $z E D A$ classification by statements interaction, $F(5,16392)=85.28, p<$ .001 (Figure 6e). "Believed" TasT responses were more likely than "believed" FasF responses, $t(16392)=18.63, p<.001, d=.69,95 \%$ CI $[.61, .77]$; and "guessed" FasF responses were more likely than "guessed" TasT responses, $t(16392)=8.96, p<.001, d=.36,95 \%$ CI $[.28, .43]$. In support of hypothesis 5, "believed" FasT errors were more likely than "believed" TasF errors, $t(16392)=8.37, p<.001, d=.48,95 \%$ CI $[.40, .55]$; and "guessed" FasT errors were not significantly different from "guessed" TasF errors, $t(16392)=1.89, p=.059$ (Figure 6e). This categorization based on zEDA magnitudes indicates that most FasT errors are believed memory mistakes and are not simply due to uncertainty followed by a guessing true bias. There was also a two-way interaction between $z E D A$ classification and group, $F(5,16392)=10.85, p<.001$ (Figure 6e). Older adults were more likely to produce "believed" veridical errors (i.e., TasF and FasT) than younger adults, $t(16392)=3.42, p=.001, d=.29,95 \% \mathrm{CI}[.21, .36]$; and younger adults were more likely to produce "guessed" hits (i.e., TasT and FasF) than older adults, $t(16392)=5.94, p<.001, d=.23,95 \%$ CI $[.16, .31]$. Finally, there was a three-way interaction observed between $z E D A$ classification, statements, and group, $F(5,16392)=7.09, p<.001$ (Figure 6e). Older adults were more likely to produce "believed" TasT responses than younger adults, $t(16392)=3.22, p=.001, d=.18,95 \% \mathrm{CI}[.07, .28]$; and younger adults were more likely to produce "guessed" TasT responses than older adults, $t(16392)=3.58, p<.001, d=.20,95 \%$ CI $[.08, .30]$. Younger adults were more likely to produce both "believed" FasF responses, $t(16392)=3.52, p<.001, d=.19,95 \%$ CI $[.08, .30]$, and "guessed" FasF responses, $t(16392)=$ 
$5.09, p<.001, d=.27,95 \% \mathrm{CI}[.17, .38]$, than older adults. In support of hypothesis 6 , older adults were more likely to produce both "believed" FasT errors, $t(16392)=6.96, p<.001, d=$ $.43,95 \%$ CI $[.32, .54]$, and "guessed" FasT errors, $t(16392)=2.62, p=.009, d=.27,95 \% \mathrm{CI}$ $[.18, .39]$, than younger adults (Figure 6e). Notably, there were no significant differences between younger and older adults for "believed" TasF errors, $t(16392)=.19, p=.846$, and for "guessed" TasF errors, $t(16392)=.08, p=.935$.

\section{Discussion}

Study 3 contrasted imbalanced, narrative-based and balanced, list-based false information paradigms' results using developmental populations. Across the younger and older adult participants, electrodermal activity recorded during true/false recognition memory tests revealed an ordinal pattern corresponding to the answer provided: true responses with the highest magnitude zEDA, false responses with middle magnitude zEDA, and never seen responses with the lowest magnitude zEDA (Figure 6c, Supplementary Figure 3). Given the limitations to determine "believed" and "guessed" memory responses using MPT model analyses that rely only on behavioral outcomes and cognitive assumptions, we reassigned the true/false recognition memory results into "believed" or "guessed" bins based on zEDA magnitude (Figure 6e, Supplementary Figure 4). These analyses strongly suggest misremembering false information as true (i.e., FasT error) is not due to an increased "guessing" true bias (Nadarevic \& Erdfelder, 2013, 2019; Street \& Kingstone, 2017; Street \& Richardson, 2015), but is often in fact a "believed" memory error. When both younger and older adults failed to correctly remember explicitly-labeled false information as false on either the DJT or HLT, they often produced FasT errors that we categorized as "believed" because their zEDA magnitude was similar to when these participants correctly remembered explicitly-labeled true information as true (i.e., TasT 
response; Figure 6c, Supplementary Figure 3). Older adults (under full attention) were particularly poor at correctly remembering explicitly-labeled false information as false (Figure 6a; Table 2; Chen, 2002; Chen \& Blanchard-Fields, 2000; Skurnik et al., 2005), and this failure resulted in increased "believed" FasT errors relative to younger adults on our imbalanced, narrative-based false information paradigm (Figure 6e).

Beyond the true/false recognition memory test results, older adults were also influenced by the designed direction of the explicitly-labeled false statements in their trait judgments more than younger adults on the DJT (Figure 6b; Chen, 2002). That is, older adults' social judgments of the protagonists in the DJT were altered by the explicitly-labeled false statements in the stories. While the "over-compensation effect" was again seen in younger adults, it was absent in the older adult trait judgments. Older adults were poor at using the false cues in the stories to moderate their trait judgments of the protagonists relative to younger adults. Moreover, in concordance with Study 2, Study 3 provided evidence that individuals swayed by the explicitlylabeled false cues also commit more FasT errors on the DJT true/false recognition memory test. Thus, individuals with greater FasT errors often behave in a way on trait judgments that is consistent with the truth of information that was explicitly-labeled as false in the DJT stories. Importantly, Study 3 also provided evidence suggesting multinomial processing tree (MPT) models offer unreliable conclusions to true/false recognition memory test results. In the same participants, MPT models supported belief-default conclusions for imbalanced, narrativebased false information paradigm data (DJT); but MPT models supported Cartesian conclusions for balanced, list-based false information paradigm data (HLT). The DJT produced increased FasT errors relative to TasF errors (Figure 6a; see also Table 2; Chen, 2002; Chen \& BlanchardFields, 2000; Gilbert et al., 1993; Pantazi et al., 2018), whereas the HLT produced equivalent 
FasT and TasF error rates (Figure 6d; Gilbert et al., 1990; Pantazi et al., 2018). Indeed, narrative-based false information paradigms that present equal numbers of true and false statements during the learning phase also produce increased FasT errors relative to TasF errors (Experiment 3, Pantazi et al., 2018). These error rates tend to heavily impact goodness-of-fit of the MPT models and thus the cognitive conclusions gleaned from them. Thus, it is likely that transparent base-rate information during list-based false information paradigms (relative to more obscure base-rate information during narrative-based false information paradigms) promote biased guessing strategies and keep different identification responses and errors at similar rates (see Introduction for Assumption 4 in results of Nadarevic \& Erdfelder, 2013) ${ }^{23}$. Thus, because MPT models only use behavioral results and cognitive assumptions to estimate guessing rates, MPT models should not be solely relied on to distinguish between "believed" and "guessing" rates in the true/false recognition memory tests. MPT models are not the royal road to delineating which responses are "believed" or "guessed". However, Study 3 also offered an independent metric which may be used to adjudicate "believed" and "guessed" responses: electrodermal activity.

The ordinal pattern of zEDA magnitude to true, false, and never seen responding was robust across participants and across false information paradigms (Figure 6c, Supplementary Figure 3). zEDA clearly did not correspond to correct responding as correct rejections (NSasNS) and correctly identifying false information as false (FasF) had reliably reduced zEDA magnitudes relative to correctly identifying true information as true (TasT). Moreover, given that effortful search processes would produce increase response latencies relative to effortless

\footnotetext{
${ }^{23}$ Another possibility is that participants actually switch between a Cartesian and a belief-default procedure according to the paradigm (e.g., Hasson et al., 2005). However, see Mandelbaum (2014) for a strong rebuttal to this prospect.
} 
search processes, zEDA also likely did not correspond with increased cognitive resource allocation for the engagement of an effortful search (e.g., Morris et al., 2008) as correct responses with longer response latencies (i.e., FasF) had reliably reduced zEDA magnitudes relative to correct responses with shorter response latencies (i.e., TasT; see Supplementary Materials for Study 3 reaction time analyses). Moreover, errant responding did not uniformly result in increased zEDA magnitudes as may be expected if zEDA magnitudes corresponded with increased cognitive resource allocation. Rather, we suggest the observed autonomic reactivity constitutes the source of epistemic emotions (Fiacconi et al., 2017) associated with outcomes of an individual's memory search. In the belief-default perspective, where true information resides in memory without associated "true tags" (Figure 1), searching and successfully recovering a proposition represented as true (i.e., TasT) may elicit a covert "aha" emotion which was indexed as a high EDA magnitude (see Introduction section on illusory truth, familiarity, and "aha"). However, for a foil proposition that was not represented in memory, a search that fails to recover a similar remembered proposition (i.e., NSasNS) does not elicit a covert "aha" emotion and is indexed as a low or near baseline EDA magnitude. Finally, if false information resides in memory with an associated "false tag" in accordance to the belief-default perspective, searching and successfully recovering both the proposition and its affixed false tag (i.e., FasF) may elicit a different covert epistemic emotion with a unique, middle EDA magnitude. We suggest this is a negative epistemic emotion and when expressed consciously, i.e., the phenomenological experience of dissonance or wrongness (Asp \& Tranel, 2013; Elkin \& Leippe, 1986).

Our rationale for characterizing high EDA magnitudes as "aha" emotions and not familiarity emotions (see illusory truth effect description in the Introduction; Morris et al., 2008) 
is that EDA magnitudes were not consistent across all hits (i.e., TasT and FasF responses). Presumably, explicitly-labeled false propositions should be as "familiar" as explicitly-labeled true propositions (i.e., each were previously seen one time), and yet FasF EDA magnitudes were reliable reduced relative to TasT EDA magnitudes (Figure 6c, Supplementary Figure 3). High EDA magnitudes were quite specific to true responding and "middle" EDA magnitudes were specific to false responding. Thus, familiarity is an inadequate designation for high EDA magnitudes that denote truth during true/false recognition memory tests. "Aha" emotions with more direct associations to truth (Laukkonen, Ingledew, et al., 2018; Laukkonen et al., 2020) are more apt for the high magnitude EDA results of Study 3.

If this epistemic emotion perspective of the EDA results is correct, it enables an independent metric to determine the percentage of "believed" and "guessed" responses in true/false recognition memory tests. Namely, misses (TasNS and FasNS) have similar zEDA magnitudes as correct rejections (NSasNS) because a memory search failed to recover a similar remember proposition (Figure 6c, Supplementary Figure 3). Moreover, if a participant performs a memory search that fails to recover a similar remember proposition, but then guesses correctly, their zEDA should be reduced relative to either "believed" TasT or FasF responding. For example, if the explicitly-labeled true proposition Bill mowed his lawn is presented to a participant, and during the true/false recognition memory test the participant fails to retrieve Bill mowed his lawn with a memory search. Although the participant does not know the correct answer, they simply guess true. In this scenario, the participant would produce the correct behavioral response (TasT) with a low zEDA. Interestingly, this EDA analysis may inform "believed/guessed" status for other responses such as FasT errors. For instance, if the explicitlylabeled false proposition Sam stole $\$ 1000$ was presented to a participant, and during the 
true/false recognition memory test the participant did retrieve the proposition Sam stole $\$ 1000$ but did not successfully retrieve its associated false tag. In the belief-default and FTT perspective (Asp \& Tranel, 2013), searching and recovering a proposition without an associated false tag would elicit a covert "aha" emotion which is indexed as a high EDA magnitude. In this scenario, the participant would produce a FasT error with a high zEDA reflective of "believed" true responding.

This hypothesis may also yield an explanation for the DJT zEDA differences in true responding seen in the all statements mixed-effects model. While FasT $(M=.55, S D=.88)$ and NSasT ( $M=.40, S D=1.06)$ zEDA magnitudes were consistently greater than zEDA magnitudes associated with false responding $(M=-.21, S D=.61)$, they were significantly reduced relative to TasT responding $(M=.73, S D=1.05$; Figure $6 \mathrm{c})$. If the epistemic emotion perspective is valid, then lower zEDA in FasT and NSasT errors may indicate increased failed memory searches followed by guessing relative to TasT responding. Our post-hoc "believed" versus "guessed" analysis examined zEDA magnitudes of hits and veridical errors in detail.

We used this epistemic emotion perspective logic to post-hoc recategorize TasT, FasF, TasF, and FasT responses into "believed" or "guessed" bins based on zEDA magnitude (Figure 6e, Supplementary Figure 4). Of note, across both younger and older adults in the DJT and HLT "believed" FasT errors were more common than "guessed" FasT errors, "believed" TasF errors, and "guessed" TasF errors. These data are in opposition to the increased guessing true bias argument challenging belief-default models (Nadarevic \& Erdfelder, 2013, 2019; Street \& Kingstone, 2017; Street \& Richardson, 2015).

One limitation to Study 3 is the use of the post-hoc analyses to identify "believed" and "guessed" true/false recognition memory test responses. Here, the statistical analyses were 
specified after the data had been seen and the ordinal EDA pattern to responding was identified. Future studies replicating this effect should a priori hypothesize these divergent outcomes. Another limitation in the post-hoc analysis of Study 3 was the difference in bin size between "believed" true responses and "believed" false responses. Bin size for false responses was capped with both minimum and maximum values, whereas bin size for true responses was only capped with a minimum value. This may result in unequal probabilities of true versus false "believed" categorization. However, in the comparison of the critical "believed" FasT versus "guessed" FasT error both bin sizes were capped by only one value. Thus, it is unlikely that bin size impacted the "believed" versus "guessed" FasT error analysis.

Another important limitation to note is the lack of self-reported ratings of emotions during the true/false recognition memory tests in Study 3. One might expect increased positive emotional valence ratings relative to true responding (and high zEDA magnitudes) and increased negative emotional valence ratings relative to false responding (and middle zEDA magnitudes). However, since the epistemic emotion hypothesis delineates these experiences as "covert" (Bechara et al., 1997; Damasio, 1994), i.e., below conscious awareness, self-reported ratings of emotional valence may not vary as a product of true and false responding to true/false recognition memory tests. Indeed, peak EDA magnitudes for these effects are small relative to traditional SCR measurements (see Morris et al., 2008) with some reliable measurements below $.02 \mu \mathrm{S}$.

In accordance with Studies 1 and 2, the DJT data provided evidence of FFABB as participants produced increased incorrect false responses to foil statements that were similar and mutually exclusive to extant true mental information $\left(\mathrm{NS}^{\neq t} \mathrm{asF}\right)$ relative to foil statements that were similar but not mutually exclusive to true mental information $\left(\mathrm{NS}^{\sim \mathrm{t}}\right.$ asF) and foil statements 
not similar to extant mental information $\left(\mathrm{NS}^{\varnothing}{ }_{\mathrm{asF}}\right.$; Figure $\left.6 \mathrm{a}\right)$. While we did not find strong evidence that older adults committed the FFABB more than younger adults, the "false" false alarm parameter for foil statements with high mutual exclusivity to true information $(z \neq t)$ was increased in older adults relative to younger adults in our DJT MPT model with belief-default assumptions.

Given the epistemic emotion hypothesis of the EDA results, the FFABB offers an additional insight to the covert epistemic emotion associated with false responding. Remarkably, zEDA magnitudes are equivalent between correct FasF responding and $\mathrm{NS}^{\neq \mathrm{t}}$ asF errors (see Supplementary Materials for a non-collapsed foil zEDA mixed model analysis; Supplementary Figure 2). The FFABB is produced by mistakenly answering that the proposition was false relative to one's current beliefs rather than whether the proposition was remembered with an affixed false tag. Thus, the act of immediate falsification of some idea elicits similar zEDA magnitudes and potentially an identical covert epistemic emotion as successfully searching memory for a false idea. Further evidence is provided by $\mathrm{NS}^{\sim \mathrm{f}}$ asF error zEDA magnitudes which are also equivalent to FasF and $\mathrm{NS}^{\neq \mathrm{t}}$ asF error zEDA magnitudes (Supplementary Materials). $\mathrm{NS}^{\sim \mathrm{f}}$ foils are similar to provided false information and zEDA reflects a "successfully" searched false proposition in memory which was never provided in the original story. Thus, there is some evidence to suggest that the "middle" zEDA response associated with false responding is an automatic, covert epistemic emotion elicited when novel ideas are inconsistent with other extant mental ideas (Asp \& Tranel, 2013). We suggest this epistemic emotion when expressed consciously is the negative feeling of dissonance or wrongness (Gawronski \& Strack, 2004; Harmon-Jones, 2000). 
Moreover, "middle" zEDA magnitudes for NS"tasF errors (Supplementary Figure 2) also offer evidence that high zEDA magnitudes (i.e., covert "aha" emotions) are not the theorized "true tags" of the Cartesian belief model. All naïve propositions (including $\mathrm{NS}^{\ddagger \mathrm{t}}$ foil propositions) must be empowered by a "truth tag" in the Cartesian belief model (Gilbert, 1993) for extant mental information consistent with the truth of the naïve proposition to be elicited. Clearly, high zEDA magnitudes are not observed when the representation of naïve propositions elicit extant mental information consistent with truth of the proposition (i.e., FFABB). Rather, "middle" zEDA magnitudes are observed which are consistent across all false responding. Thus, while high zEDA magnitudes (i.e., covert "aha" emotions) may signal truth following memory searches, they are not common following naïve propositional representation, and are not the theoretical "true tags" of the Cartesian belief model.

If the epistemic emotion explanation of the EDA findings is correct, EDA magnitudes should be specific to propositions considered by the participant to be actually true or false. According to this hypothesis perceived veridical identification is a requisite for the ordinal EDA pattern during recognition memory tests. Thus, propositional content, per se, without truelfalse labeling should not produce a similar ordinal EDA pattern. That is, source memory recognition tests that do not group items from different sources into true or false categories should not show reliable zEDA differences between successful source identification categories during recognition memory tests. We suggest that veridical identification and source identification are distinct processes (see Introduction for Assumption 1 in results of Nadarevic \& Erdfelder, 2013). Indeed, there is a clear memory performance advantage when participants encode the same stimuli from true/false categories than from two arbitrary sources (Begg et al., 1992; Nadarevic \& Erdfelder, 2013). In Study 4, we sought to examine the potential differences between 
veridical identification and non-veridical source identification. We used nearly identical stimuli of the DJT and HLT but asked participants to remember the non-veridical sources and correctly classify to which source the stimuli belonged during the recognition memory tests. For instance, in the Source Memory DJT (SM-DJT) we did not instruct the participants that information in white text were facts and information in red text were fictions, but instead told them they would need to remember during a later recognition memory test which statements were presented in white or red text. If zEDA magnitudes are reflective of epistemic emotion endemic in true and false memory retrieval processes, then zEDA magnitudes should not differ between correctly identifying white information as white responses (WasW) and correctly identifying red information as red responses (RasR).

Beyond a zEDA magnitude analysis of source memory, Study 4 also enabled verification of three DJT assumptions made in the original design: 1) The DJT was designed to be imbalanced and to obscure base-rate information during the learning phase. If the DJT was perceived as imbalanced by participants, the SM-DJT should produce increased white guesses during source errors (i.e., red information misremembered as white, RasW, relative to white information misremembered as red, WasR). 2) Since the SM-DJT does not include explicitlylabeled false cues, false cue moderation of trait judgments in Studies 1-3 can be clarified by comparison to the SM-DJT trait judgments where all information in the learning phase is considered true. 3) Finally, the SM-DJT may offer evidence that increased $\mathrm{NS}^{\neq t}$ asF errors on the original DJT are a product of answering false against one's current beliefs rather than the memory of a proposition with an affixed false tag. During SM-DJT white/red recognition memory tests, the FFABB should be abolished and foil statements that are similar and mutually exclusive to extant white mental information $\left(\mathrm{NS}^{\neq \mathrm{w}}\right)$ should have similar rates of incorrect red 
responses as foil statements that are similar but not mutually exclusive to white mental information $\left(\mathrm{NS}^{\sim \mathrm{w}}\right)$ and foil statements that are not similar to extant mental information $\left(\mathrm{NS}^{\varnothing}\right)$; i.e., $\mathrm{NS}^{\neq \mathrm{w}}$ asR $\sim \mathrm{NS}^{\sim \mathrm{w}}$ asR $\sim \mathrm{NS}^{\varnothing}$ asR.

\section{$\underline{\text { Study } 4}$}

To achieve these aims we gave younger adults a source memory version of the DJT (SMDJT) and the HLT (SM-HLT), and recorded EDA during the tasks. Rather than have participants remember true and false information based on text color cues (white = true; red = false) as in the original DJT, the SM-DJT instructed participants to remember what propositions were presented in white or red text during the DJT stories. Memory performance was evaluated via a white/red recognition memory test following each story. During the SM-HLT participants were instructed that they would need to remember which nonsense, "abstract" terms were associated with the Hopi language to English equivalent proposition: TROP or FAVIL. Again, memory was evaluated via a trop/favil recognition memory test following each learning session. The primary aim of Study 4 was to probe EDA responses during the source memory recognition tests. Secondary aims included evaluation of original DJT assumptions and an examination of the FFABB as an explanation for increased $\mathrm{NS}^{\neq \mathrm{t}}$ asF errors during the original DJT.

Method

Overview. In all participants the SM-HLT was completed prior to the SM-DJT. During the SM-HLT participants were presented with novel propositions in a learning phase, and were explicitly signaled 2 seconds after whether the prior proposition was associated with the nonsense word TROP or the nonsense word FAVIL. Subsequently, in a testing phase, participants were presented with the original propositions (in question form) and were asked to determine whether the proposition was associated with trop, favil, or if it had never been 
presented (never seen). Several foils not presented in the learning phase were included in the trop/favil recognition memory test.

During the SM-DJT participants read four stories regarding unique protagonists that contained both explicitly-labeled white and explicitly-labeled red statements. Only the instructions were changed from the original DJT to the SM-DJT. The actual stimuli in the learning phase were identical between the two DJT versions.

Participants. A group of 23 younger adults from a community-based sample participated in this study (age $M=39.7$, age $S D=7.5$; years of education $M=17.5$, years of education $S D=$ 2.1). Nine of the participants were male and 14 were female. All participants were native English speakers and were free from intellectual disability, learning disabilities, psychiatric disease, substance abuse, and dementia. Participants gave informed consent approved by the Institutional Review Board of the University of Iowa. This study was not pre-registered prior to the research being conducted. No participants were excluded from this study post-hoc.

Stimuli and procedure. The stimuli and procedure replicated Study 3 except for the alteration of instructions in the tasks and minor changes of the HLT stimuli (i.e., TRUE to TROP and FALSE to FAVIL). EDA was recorded via the thenar and hypothenar eminences of the participants' non-dominant hand in the same manner with the same equipment as Study 3.

$\underline{S M-D J T}$. Participants were given both written and spoken instructions for the SM-DJT that differed from the original DJT (Supplementary Materials). Participants were told that "you will be given two kinds of information: WHITE and RED. Some information will be presented in WHITE type and some information will be presented in RED type. Afterward, you will be asked about which information was presented in WHITE type and which information was presented in RED type.” After reading each story, participants made trait judgments about the protagonists 
and then were given a white/red source memory recognition test regarding the story details. During the white/red recognition memory tests participants responded by pressing one of three buttons: white, red, or never seen. The stimuli itself was identical to the original DJT in Studies $1-3$.

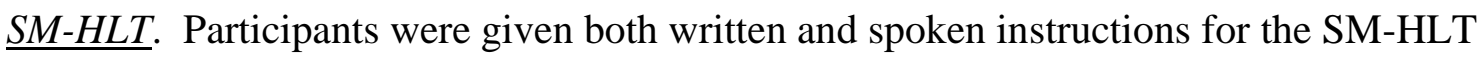
that differed from the original HLT (Supplementary Materials). Participants were told that "sometimes new terms must be associated with other foreign terms, so here you will be asked to learn new terms and associate them with other abstract terms." Participants were notified that they would need to associate the nonsense words, TROP and FAVIL, with the Hopi nouns shown during the learning phases in later recognition memory tests. TROP and FAVIL were chosen as surrogates for TRUE and FALSE due to equivalent letters, English pronounceability, and no apparent meaning. In the SM-HLT, TROP replaced every presentation of TRUE in the original HLT and FAVIL replaced every original HLT presentation of FALSE. During the SM-HLT trop/favil recognition memory tests each proposition was rephrased in the form of a question (e.g., Is a monishna is a star?) as in the original HLT. However, participants responded by pressing one of three buttons: trop, favil, or never seen. The order of the stimuli throughout the task was identical to Study 3 (see Supplementary Materials for SM-HLT results).

Study 4 hypotheses. Hypothesis 1) We hypothesized that EDA magnitudes during correct source identification in both the SM-DJT and SM-HLT will not significantly differ. In the SMDJT, correctly identifying white information as white (WasW) and correctly identifying red information as red responses (RasR) will have equivalent zEDA magnitudes. In the SM-HLT, correctly identifying trop information as trop (ToasTo) and correctly identifying favil information as favil (FvasFv) will also have equivalent zEDA magnitudes. Hypothesis 2) If the 
SM-DJT was perceived as imbalanced by participants, then RasW errors will be increased relative to WasR errors. Hypothesis 3) If the FFABB produces increased $\mathrm{NS}^{\ddagger t}$ asF errors during the original DJT, then the rate of $\mathrm{NS}^{\neq \mathrm{w}}$ asR, $\mathrm{NS}^{\sim \mathrm{w}}$ asR, and $\mathrm{NS}^{\emptyset}$ asR errors will be equivalent on the SM-DJT.

Statistical analyses. SM-DJT and SM-HLT statistical analyses closely corresponded with Study 3 without the additional group-level fixed factor (younger and older adults) in our mixed-effects models. All two- and three-way interactions of fixed factors were examined in each model. The Pearson correlation between trait judgments and FasT error rates was also omitted due to no hypothesized relationship between source errors and judgments in the SMDJT. EDA analyses were conducted in an identical manner to Study 3. See Supplementary Materials for SM-HLT trop/favil recognition memory test and SM-HLT EDA statistical approaches and data analyses.

SM-DJT old statements statistics. For the SM-DJT old statements mixed-effects model, there were two fixed factors: classification (hit, source error, miss) and statements (white, red). Intercepts and slopes for participants and recognition memory test items were added as random effects. We examined hypothesis 2 with the inclusion of a classification by statements interaction.

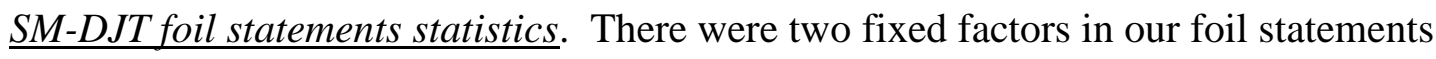
mixed-effects model: answer (correct rejection, "white" false alarm, "red" false alarm), and statements $\left(N S^{\emptyset}, N S^{\sim w}, N S^{\ddagger w}, N S^{\sim}\right)$. Intercepts and slopes for participants and recognition memory test items were added as random effects. We examined hypothesis 3 with the inclusion of an answer by statements interaction. 


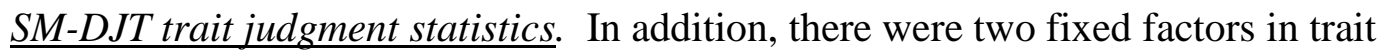
judgment mixed-effects model: red valence (positive reds, negative reds), and critical trait (cognitive assessment, moral assessment, likeable assessment). Intercepts for participants were added as random effects.

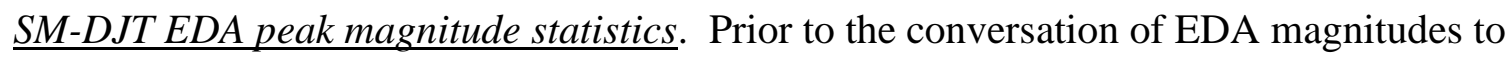
standard scores (zEDA), average EDA peak magnitudes were calculated across all participants for each type of recognition memory test behavioral response: white, red, and never seen.

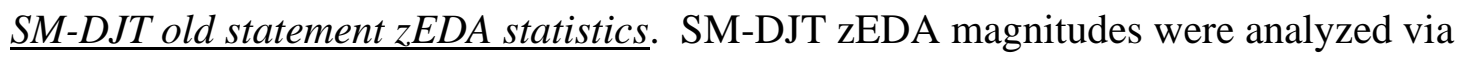
two mixed-effects models to allow for both a direct comparison of the type of response (hits/source errors/misses) but also of the answer provided (white/red/never seen). Examination of SM-DJT old statements zEDA included fixed factors classification (hit, source error, miss) and statements (white, red), which allowed a classification by statements interaction addressing hypothesis 1 . Intercepts for participants and recognition memory test items were added as random effects.

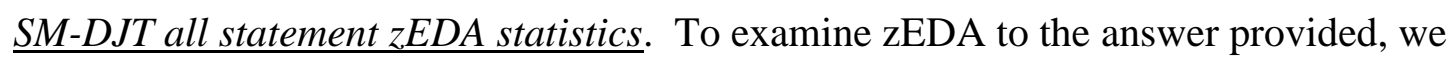
collapsed the differing foil conditions and outcomes together and included old statements for an overall analysis. Thus, our all statement zEDA mixed-effects model included fixed factors answer (white, red, never seen) and statements (white, red, foil), which allowed an answer main effect and an answer by statements interaction addressing hypothesis 1 . Again, intercepts for participants and recognition memory test items were added as random effects.

Results

SM-DJT old statements results. There was a main effect of classification, $F(2,3306)=$ $558.78, p<.001$. Old statements were more likely to be hits than source errors, $t(3306)=43.25$, 
$p<.001, d=1.84,95 \% \mathrm{CI}[1.74,1.94]$, and misses, $t(3306)=47.14, p<.001, d=2.02,95 \% \mathrm{CI}$

$[1.92,2.13]$. No significant differences were observed between misses and source errors. There was also a classification by statements interaction, $F(2,3306)=37.16, p<.001$. White statements were more correctly classified as white (WasW) than red statements correctly classified as red $(\operatorname{RasR}), t(3306)=5.45, p<.001, d=.33,95 \%$ CI $[.21, .44]$ (Figure 7a). In support of hypothesis 3, red statements were more likely misclassified as white (RasW) than white statements were misclassified as red (WasR), $t(3306)=7.37, p<.001, d=.49,95 \% \mathrm{CI}$ $[.37, .61]$ (Figure 7a).

SM-DJT foil statements results. The SM-DJT foil statements mixed-effects model found a main effect of answer, $F(2,3300)=610.27, p<.001$. Foil statements were more correctly identified as never seen (NSasNS) than misclassified as white (NSasW), $t(3300)=84.85, p<$ $.001, d=4.07,95 \%$ CI $[3.93,4.22]$ or misclassified as red (NSasR), $t(3300)=93.44, p<.001, d$ $=4.92,95 \%$ CI $[4.76,5.09]$. Foil statements were more likely to be misclassified as white $(\mathrm{NSasW})$ than misclassified as red (NSasR), $t(3300)=2.11, p=.035, d=.17,95 \% \mathrm{CI}[.08, .25]$. There was also an answer by statements interaction, $F(6,3300)=2.30, p=.033$. Foil statements not related to white nor red statements were more correctly rejected (NS $\left.{ }^{a_{a s N S}}\right)$ than foil statements similar to red statements ( $\mathrm{NS}^{\sim \mathrm{r}}$ asNS), $t(3300)=2.61, p=.009, d=.30,95 \%$ CI $[.14$, .47]. In support of hypothesis 4 , there were no significant differences between either "red" false

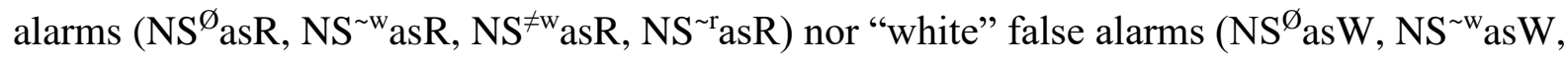
$\mathrm{NS}^{\neq \mathrm{w}}$ asW, $\mathrm{NS}^{\sim \mathrm{r}}$ asW; Figure 7a).

SM-DJT trait judgment results. No responses were excluded as outliers. Without the moderating effect of false cues, the SM-DJT produced divergent trait judgments on the valence of the red (i.e., considered true) information. There was a main effect of critical trait, $F(2,248)$ 
$=22.64, p<.001$. Likeable assessments had increased ratings relative to cognitive assessments (intelligence/clever), $M D=.98, p<.001, d=.40,95 \%$ CI [.11, .70], and moral assessments (honest/sincere), $M D=1.28, p<.001, d=.43,95 \%$ CI [.14, .72]. Critically, there was also a main effect of red valence, $F(1,248)=803.19, p<.001$. Positive reds had increased ratings relative to negative reds, $M D=4.61, p<.001, d=2.76,95 \%$ CI $[2.44,3.10]$ (Figure 7b). In addition, there was a red valence by critical trait interaction, $F(2,248)=33.05, p<.001$. Positive reds had increased ratings relative to negative reds in cognitive assessments, $M D=3.70$, $p<.001, d=2.47,95 \%$ CI $[1.94,3.03]$, moral assessments, $M D=6.48, p<.001, d=5.87,95 \%$ CI [4.96, 6.88], and likeable assessments, $M D=3.65, p<.001, d=2.18,95 \%$ CI $[1.67,2.71]$ (Figure $7 b$ ). Thus, in every trait metric when red text does not indicate a false cue, trait judgment ratings are divergent based on the proposition valence of the stories.

SM-DJT EDA peak magnitude results. Across all participants in the SM-DJT recognition memory test, EDA peak magnitudes to the answer provided included: white answering ( $M=.015$ $\mu \mathrm{S} ; S D=.021 \mu \mathrm{S})$, red answering $(M=.012 \mu \mathrm{S} ; S D=.015 \mu \mathrm{S})$, and never seen answering $(M=$ $.013 \mu \mathrm{S} ; S D=.019 \mu \mathrm{S})$.

SM-DJT old statement zEDA results. Of the total responses $11.23 \%$ were excluded as outliers. The SM-DJT old statement zEDA mixed-effects model showed a main effect of classification, $F(2,974)=4.81, p=.008$. Source errors $($ WasR, RasW) had increased zEDA relative to misses (WasNS, RasNS), $M D=.32, p=.006, d=.23,95 \% \mathrm{CI}[.05, .51]$. No significant differences were observed between hits and source errors. There was also a classification by statements interaction, $F(2,974)=16.28, p<.001$. WasR errors had greater zEDA magnitudes than RasW errors, $M D=.58, p<.001, d=.99,95 \%$ CI $[.50,1.49]$, and RasNS errors had greater zEDA magnitudes than WasNS errors, $M D=.58, p<.001, d=.89$, 
95\% CI [.45, 1.33] (Figure 7c). However, in support of hypothesis 1, no significant differences were observed between WasW and RasR zEDA magnitudes, $M D=-.05, p=.254$ (Figure 7c).

SM-DJT all statement zEDA results. Replicating the statistical methods from Study 3, we again collapsed the differing foil conditions together and included old statements for an overall SM-DJT zEDA mixed-effects model. Of the total responses $10.46 \%$ were excluded as outliers. The SM-DJT all statement zEDA mixed-effects model showed a main effect of answer, $F(2$, $1968.00)=5.67, p=.004$. Never seen responding (WasNS, RasNS, NSasNS) had reduced zEDA magnitudes relative to white responding (WasW, RasW, NSasW), $M D=-.15, p=.042, d$ $=.06,95 \% \mathrm{CI}[0, .16]$, and $r e d$ responding $(\operatorname{RasR}, \mathrm{WasR}, \mathrm{NSasR}), M D=-.26, p=.006, d=.16$, $95 \%$ CI $[.04, .27]$. Moreover, in further support of hypothesis 1, no significant differences were observed between white responding (WasW, RasW, NSasW) and red responding (RasR, WasR, NSasR), $M D=-.11, p=.517$. Thus, zEDA magnitudes during the white/red recognition memory tests did not correspond to the answer provided in an ordinal nature as was observed during true/false recognition memory tests (see Study 3). There was also a classification by statements interaction, $F(4,1968.00)=8.56, p<.001$ (Figure 7c). NSasW errors had greater zEDA magnitudes than WasW responses, $M D=.29, p=.013, d=.44,95 \% \mathrm{CI}[.13, .75]$, and RasW errors, $M D=.38, p=.003, d=.59,95 \% \mathrm{CI}[.23, .96]$. WasR errors had greater zEDA magnitudes than RasR responses, $M D=.44, p=.007, d=.66,95 \%$ CI [.22, 1.11], and NSasR errors, $M D=.50, p=.047, d=.57,95 \%$ CI [0, 1.23]. Finally, WasNS errors had reduced zEDA magnitudes relative to RasNS errors, $M D=-.58, p<.001, d=.89,95 \%$ CI [.45, 1.33], and NSasNS responses, $M D=-.39, p<.001, d=.64,95 \%$ CI $[.34, .93]$. These divergent interaction findings offer additional support that zEDA magnitudes during the white/red recognition 
memory test did not correspond to the robust zEDA ordinal pattern of answering in true/false recognition memory tests.

\section{Discussion}

Study 4 examined EDA responses during non-veridical, source memory recognition tests using the identical base stimuli from Study 3. Across both the SM-DJT and SM-HLT (see Supplementary Materials), participants did not show reliable zEDA magnitude differences to correct source memory responding (Figure 7c, Supplementary Figure 6). Thus, the zEDA magnitude differences observed in Study 3 cannot be due to the informational content of the propositions in the DJT and HLT, per se, since the propositions and their presentation to participants were the same in Study 3 and 4. Rather, we suggest that veridical identification (classifying a proposition as true or false) is qualitatively distinct from non-veridical, arbitrary source identification (classifying a proposition as belonging to category " $\mathrm{A}$ " or "B") as covert epistemic emotion markers clearly demarcate the mnemonic approaches. A lack of EDA difference between source responding was evident in the SM-DJT and SM-HLT EDA peak magnitude results as the means for type of response in both tasks were equivalent.

While some errors did correlate with increased zEDA magnitudes relative to other responses in the SM-DJT (e.g., WasR errors), correct source responding (WasW, RasR, NSasNS) did not result in the robust ordinal zEDA pattern of correct veridical responding (TasT $>$ FasF $>$ NSasNS) present in Study 3. Increased zEDA magnitudes following errors on the SMDJT may be the product of arousal due to increased effortful search processes during recollection (e.g., Morris et al., 2008). However, this hypothesis remains tenuous as other errors did not show this increased zEDA pattern including RasW in the SM-DJT, as well as ToasFv and FvasTo in the SM-HLT (Supplementary Materials). 
On the SM-DJT zEDA magnitudes for never seen responses were reduced relative to white responses and red responses. This result is consistent with the epistemic emotion hypothesis proposed in Study 3 as white and red responding are often the product of a "successful" memory search relative to never seen responding which is often the product of a failed memory search. In this perspective increased EDA is observed after a perceived "successful" memory search. Nevertheless, this EDA pattern was not replicated in the SM-HLT results and the effect sizes were tellingly reduced relative to the commensurate original DJT zEDA results: white $>$ never seen, $d=.06$; red $>$ never seen, $d=.16$; true $>$ never seen, $d=1.61$; and false $>$ never seen, $d=.64$. Thus, if there is a reliable increase in zEDA magnitudes for source responding relative to never seen responding, it is meager relative to zEDA magnitudes for veridical responding.

Study 4 also provided additional evidence that the original DJT was perceived by participants as imbalanced. RasW source errors were increased relative to WasR source errors (Figure 7a). Thus, DJT made it less likely that participants could try to optimize performance by tuning responses toward apparent recognition memory test base-rates as in the HLT. The data suggest that in the SM-DJT participants were more likely to guess white when uncertain than to answer equivalent white, red, and never seen responses (see potential metacognitive guessing strategies in the Introduction, Assumption 4 of Nadarevic \& Erdfelder, 2013).

Since there were no false cues in the SM-DJT, trait judgments reflected all information in the DJT stories as true (Figure 7b). Clearly false cues moderated trait judgments in Studies 1-3 in both the positive and negative valence direction as well as across the different trait assessments (Figures 3b, 4b, 6b) as SM-DJT trait judgments reflect the most extreme judgments in the designed direction across studies. Thus, participants in the original DJT acted as if red 
information was false and modified their trait judgments accordingly. Participants that modified their trait judgments according to false cues less often produced more FasT errors (Study 2, $r=$ .54 ; Study $3, r=.68$ ). Therefore, FasT errors may be an important metric for identifying behavior consistent with false information, i.e., credulity, on the individual difference level.

Finally, the SM-DJT provided evidence that increased $\mathrm{NS}^{\neq \mathrm{t} a s F}$ errors on the original DJT was not a product of proposition familiarity or similarity. When participants were labeling foils similar but mutually exclusive to white mental information $\left(\mathrm{NS}^{\neq \mathrm{w}}\right)$, they did not produce increased red errors (NS ${ }^{\neq \mathrm{w}}$ asR) relative to other foils (i.e., $\mathrm{NS}^{\sim \mathrm{w}}$, $\mathrm{NS}^{\varnothing}$; Figure 7a). These SMDJT foils had the exact same propositional content as the original DJT. Thus, increased false responding to $\mathrm{NS}^{\ddagger \mathrm{t}}$ foils (Figures $3 \mathrm{a}, 4 \mathrm{a}, 6 \mathrm{a}$ ) is likely a product of incorrectly answering false against one's current beliefs rather than mistakenly activating a remembered false proposition. This data supports the FFABB explanation of increased $\mathrm{NS}^{\neq \mathrm{t}} \mathrm{asF}$ errors and indicate that incoming naïve propositions are indeed ballistic information that automatically elicit extant mental representations including background assumptions and mutual exclusivity information in accordance with belief-default models.

\section{General Discussion}

The present research demonstrates that believing is default during proposition comprehension (Gilbert, 1991). The results of four studies indicated that the implications from the truth of an incoming, naïve foil proposition will bias responding during recognition memory of propositions as automatic, extant, and true mental information comparison impairs performance (Figure 3a, 4a, 6a, 7a). Moreover, unique electrodermal activity signals robustly corresponded to propositions considered true and false by the participants and offered psychophysiological evidence against an increased guessing true bias challenging belief-default 
models (Nadarevic \& Erdfelder, 2013, 2019; Street \& Kingstone, 2017; Street \& Richardson, 2015). In addition, our data replicate multiple studies showing that correctly identifying false information takes more time than correctly identifying true information (e.g., Carpenter \& Just, 1975; Gilbert et al., 1990; Gough, 1965; Harris et al., 2008), a finding consistent with the beliefdefault model's additional step of false tag identification following propositional recognition (Figure 2b). Finally, our studies undermine anti-belief-default model evidence deduced from multinomial processing tree (MPT) statistical models (Nadarevic \& Erdfelder, 2013, 2019) as cognitive architecture conclusions depend on the type of false information paradigm and its responding base-rate transparency. In sum, these studies offer broad support for the central assertion in belief-default models: believing is inexorable during comprehension.

In true/false recognition memory tests, participants consistently demonstrated a "false" false alarm belief bias (FFABB) when answering foil information that was similar to, and mutually exclusive with, previously learned true information (Figure 3a, 4a, 6a). We classify this as a "belief bias" (Sa et al., 1999; Stanovich, 2003) in that prior knowledge acted to override the logic of the current problem (i.e., memory recognition). The false responses to foils mutually exclusive with true information $\left(\mathrm{NS}^{\neq \mathrm{t}}\right.$ asF error) is not due to reduced familiarity of foil statements relative to old statements (Pantazi et al., 2018) as foil statements not similar to true nor false statements did not produce increased false responding ( $\mathrm{NS}^{\varnothing}$ asF errors). Moreover, the $\mathrm{NS}^{\ddagger \mathrm{t}}$ asF error is not likely the result of increased propositional familiarity or fluency, per se: 1) the same stimuli did not produce increased surrogate red errors when veridical assessment was absent from the task design ( $\mathrm{NS}^{\neq \mathrm{w}}$ asR errors, Figure 7a) and 2) false responses to foils similar but not mutually exclusive with true information $\left(\mathrm{NS}^{\sim \mathrm{t}}\right.$ asF error) were reliable reduced relative to 
$\mathrm{NS}^{\neq \mathrm{t}} \mathrm{asF}$ errors ${ }^{24}$. Rather, the evidence suggests $\mathrm{NS}^{\neq t} \mathrm{asF}$ errors are "believed" false responses as they elicit equivalent EDA magnitudes as false statements correctly classified as false (FasF; see Supplementary Figure 2). Thus, upon encountering a naïve proposition, individuals will process the implications from the truth of the proposition, even when that processing is detrimental to task performance.

While the FFABB supports belief-default models, it also implies a quick rejection of information inconsistent with extant mental beliefs. Although there is some confusion in the literature on this point (Mercier, 2017; Richter et al., 2009; Sperber et al., 2010), belief-default models are not mutually exclusive with fast and efficient epistemic vigilance perspectives ${ }^{25}$. Instead, belief-default models assert that the stance of incoming information is true and if no extant mental information is activated to contradict the new information, it will continue to be considered true (Asp \& Tranel, 2013). Upon comprehension of propositions antithetical to "strong" background beliefs (Richter et al., 2009), rejection of naïve propositions may occur quickly and efficiently. In the belief-default perspective, epistemic vigilance functioning is dependent on the timely activation of mental information that may contradict an incoming proposition. Individuals who fail to activate contradictory mental information ${ }^{26}$ or lack the information entirely will continue to consider the naïve proposition as true. Thus, individuals will not be credulous to all incoming propositions but only to those that do not activate contradictory mental information. This view may also offer an explanation for why older adults

\footnotetext{
${ }^{24}$ Thus, the activation of corresponding references in memory (i.e., nodes), per se, does not elicit increased false responding. Instead the coherence of the foil statement (relative to extant true mental information) drawn from its truth implications evokes false responding (see also Unkelbach \& Rom, 2017 for a referential theory of falsification with belief-default perspective assumptions).

${ }^{25}$ For a rationale of why belief-default perspectives need a vigilant conflict detector, see Pennycook et al. (2019).

${ }^{26}$ Of course, having contradictory mental information stored in memory is not sufficient to reject a naïve proposition. One must activate the contradictory mental information to induce rejection. Belief-default perspectives entail fragmented or compartmentalized perspectives of the mind (Bendana \& Mandelbaum, in press; Egan, 2008).
} 
did not show a reliable increase in $\mathrm{NS}^{\neq \mathrm{t}}$ asF errors relative to younger adults (Study 3, Figure 6a). While older adults may incorrectly answer false when contradictory mental information is activated more than younger adults, older adults may not as quickly and efficiently activate contradictory mental information when comprehending $\mathrm{NS}^{\neq \mathrm{t}}$ foils. Future work will be needed to clearly delineate the mechanisms of FFABB in developmental populations.

The current research weakens anti-belief-default evidence derived from MPT modeling (Nadarevic \& Erdfelder, 2013, 2019). Using the same participants, MPT models produced divergent belief model conclusions depending on the type of false information paradigm: narrative-based or list-based (Study 3). Under no cognitive load, list-based false information paradigms often do not produce differences between veridical errors (i.e., FasT TasF, Gilbert et al., 1990; Pantazi et al., 2018), whereas in narrative-based false information paradigm veridical errors are often unequal (i.e., FasT > TasF, Table 2, Chen, 2002; Chen \& Blanchard-Fields, 2000; Gilbert et al., 1993; Pantazi et al., 2018). Since these error rates heavily impact goodnessof-fit of the MPT models, their divergence indicates different cognitive architecture conclusions based on the presentation mode of the false information paradigm. Rather than suggest the untenable hypothesis that presentation mode somehow influences cognitive architecture, we favor the increased transparent base-rate perspective in list-based false information paradigms that likely promote biased guessing strategies, which maintain identification responses and errors at similar rates. Because MPT modeling only uses behavioral results and cognitive assumptions to estimate guessing rates, memory tasks with transparent base-rate information may be subject to metacognitive guessing strategies that lead to errant cognitive architecture conclusions (e.g., Batchelder \& Batchelder, 2008; Kuhlman \& Bayen, 2016). Taken together, this work suggests 
current approaches to MPT modeling may offer unreliable conclusions for belief fixation models.

However, the ordinal pattern of EDA magnitudes to true, false, and never seen responding potentially reveal individual memory search insights independent of behavioral results and cognitive assumptions. While EDA measures are often sensitive to a wide variety of stimuli, including novelty, familiarity, intensity, significance, cognitive effort, potential threat, reward, emotional valence, and even the omission of an expected stimulus (Brown et al., 2012; Critchley, 2002; Dawson et al., 2000; Siddle, 1991), the robust EDA pattern seen across all participants and across narrative- and list-based true/false recognition tests (Study 3) curtails some potential explanations for the increases in arousal when answering true and false. First, EDA clearly did not correspond to stimuli, per se, but instead tended to accompany the type of behavioral response. Thus, explanations such as stimulus novelty, stimulus significance, and the stimulus emotional valence may be dismissed as likely candidates. While the DJT contained propositions with positive and negative emotional valence (e.g., amoral actions from the protagonist John), Study 4 (without veridical assessment) showed no robust EDA pattern with the identical DJT stimuli. Moreover, the ordinal EDA pattern was observed during the HLT (Supplementary Figure 3) which did not contain stimuli with varying emotional valence. As examined in the Study 3 Discussion, EDA did not correspond to objectively correct responding as correctly identifying true information as true had reliably greater EDA magnitudes than correctly identifying false information as false, which had reliably greater EDA magnitudes than correctly identifying foil information as never seen (TasT > FasF > NSasNS, Figure 6c, Supplementary Figure 3). This ordinal EDA pattern was not observed in Study 4 (WasW RasR; ToasTo $\sim$ FvasFv $\sim$ NSasNS, Figure 7c, Supplementary Figure 6). Thus, while not 
examined with a direct assessment, the epistemic emotion of confidence, per se, toward a correct response is also an unlikely possibility to explain the ordinal EDA pattern. Also considered in the Study 3 Discussion was the likelihood of EDA corresponding to increased cognitive effort. Of course, errant responding did not uniformly result in increased EDA magnitudes (Figure 6c, Supplementary Figure 3) which might be expected if EDA corresponding with increased cognitive resource allocation. Indeed, since errors on the DJT and HLT (Study 3) tended to be reflective of the answer provided (true, false, or never seen), it is also unlikely that the epistemic emotion of uncertainty may be able to account for the ordinal EDA pattern.

Instead, we suggest the ordinal EDA pattern is best explained as autonomic reactivity that constitutes the source of epistemic emotion (Fiacconi et al., 2017) associated with the perceived outcomes of a memory search. Specifically, we hypothesize the high EDA magnitude associated with true responding and the middle EDA magnitude associated with false responding represent distinct epistemic emotions reflective of different outcomes following a memory search. When an individual searches and recovers a stimulus-matched proposition from memory that does not have an affixed false $\operatorname{tag}^{27}$, a covert "aha" emotion is elicited which is indexed as a high EDA magnitude. Positive "aha" emotions may be indexed by EDA (Shen et al., 2018), and they are often associated with the resolution of an impasse during traditional insight problems (Webb et al., 2018; Weisberg, 2015). However, we hypothesize that covert "aha" emotions accompany more elementary problems including signaling correct memory recovery during retrieval processes. EDA is sensitive to familiarity (Morris et al., 2008; Plouffe \& Stelmack, 1984; Stelmack et al., 1983; Tranel et al., 1985), i.e., previously seen items; and positive mood induction increases familiarity (Garcia-Marques et al., 2004). However, EDA is pronounced

\footnotetext{
${ }^{27}$ Note that false tags may not be discrete, see the Introduction for a description of the FTT's continuous tagging process (Figure 1).
} 
only when task demands require recognition (Shearer \& Mikulka, 1996). In addition, successfully recollecting items during more difficult tasks relative to easy tasks (i.e., creating more "impasses") elicits increased EDA (Shearer \& Mikulka, 1996). Thus, we suggest that covert, positive "aha" emotions to successfully identified items during memory tasks is responsible for increased EDA to familiar stimuli.

When an individual searches and recovers a stimulus-matched proposition from memory that does have an affixed false tag, a different covert emotion is elicited (i.e., dissonance or wrongness) which is indexed as a middle EDA magnitude. Our rationale for the distinct covert emotion elicitation during falsification identification is that $\mathrm{NS}^{\neq \mathrm{t}}$ asF errors, which are not the product of a perceived successful proposition memory search with an affixed false tag, but instead are a product of answering false after activation of one's own true beliefs (i.e., FFABB), are associated with similar "middle" EDA magnitudes as other correctly-remembered false propositions (Supplementary Figure 2). Thus, we hypothesize the covert emotion elicited when remembering a false proposition or labeling a naïve proposition as false relative to one's own beliefs is the negative emotion of wrongness or dissonance when experienced consciously (Asp \& Tranel, 2013; Elkin \& Leippe, 1986). Finally, when an individual searches but fails to recover a stimulus-matched proposition from memory, no covert emotion is elicited and a low or baseline EDA magnitude is observed. This epistemic emotion hypothesis is consistent with affect-as-information perspectives (Damasio, 1994; Schwarz, 2012) and research indicating positive and negative emotional states have discrepant influences on truth judgments (Forgas, 2019; Koch \& Forgas, 2012).

Of note, this epistemic emotion perspective of the EDA results reflects subjective (not objective) veridical identification (Volz et al., 2017). Thus, objectively true stimuli may be 
errantly recovered with an affixed false tag and indexed with a middle EDA magnitude (i.e., a "believed" TasF error) or errantly not recovered at all (i.e., a TasNS error). Objectively false stimuli may be errantly recovered without an affixed false tag and indexed with a high EDA magnitude (i.e., a "believed" FasT error) or errantly not recovered at all (i.e., a FasNS error). Objective foil stimuli may be errantly recovered without an affixed false tag and indexed with a high EDA magnitude (i.e., a "believed” NSasT error), or errantly recovered with an affixed false tag and indexed with a middle EDA magnitude (i.e., a "believed" NSasF error).

The epistemic emotion hypothesis of the EDA results allowed an independent metric which was able to classify "believed" and "guessed" outcomes on true/false recognition memory tests. Namely, both "guessed" true and false behavioral responses will be accompanied by a low EDA as an epistemic emotion reflective of a perceived successful memory search is not elicited (see Study 3 Discussion). Thus, the epistemic emotion hypothesis enabled a post-hoc "believed" vs. "guessed" reexamination of the critical FasT errors during the DJT and HLT (Study 3). If increased FasT errors during true/false recognition memory tests do in fact represent an uncertainty state followed by an increased "guessing" true bias (Nadarevic \& Erdfelder, 2013, 2019; Street \& Kingstone, 2017; Street \& Richardson, 2015) most FasT errors should be accompanied by a low EDA magnitude reflective of a failed memory search. However, according to the EDA analysis of the DJT, we observed that in younger adults $85 \%$ of FasT errors are high EDA “believed” FasT errors, whereas in older adults 77\% of FasT errors are high EDA “believed" FasT errors (Figure 6e $)^{28}$. Thus, this analysis coupled with the strong correlations between false information swayed trait judgments and FasT errors (see also Pantazi et al., 2018) suggest most FasT errors are as believed as true propositions and may be important

${ }^{28}$ Of course, older adults make both more "believed" FasT and "guessed" FasT errors than younger adults (Figure $6 e)$ 
indices of the tendency to behave in a manner consistent with explicitly-labeled false information, i.e., credulity.

The results from Study 3 replicate and extend developmental research indicating an impairment in falsification processes in older adults over the age of 65 (Chen, 2002; Chen \& Blanchard-Fields, 2000; Skurnik et al., 2005; Swire et al., 2017). While various mechanisms for this effect have been proposed, including less efficient strategic memory processes (e.g., Prull et al., 2006) or impairments in prefrontal cortex functioning (e.g., Asp et al., 2012; Denburg et al., 2007), it is doubtless that, on average, older adults are more vulnerable to believing explicitlylabeled false information following a delay than younger adults. Our EDA analysis of DJT FasT errors indicated that for every 4 explicitly-labeled false statements, older adults would remember and believe 1 to be true (i.e., high EDA "believed" FasT errors); whereas younger adults would remember and believe 1 of 10 explicitly-labeled false statements to be true. If our epistemic EDA emotion hypothesis is correct and high EDA magnitudes reflect "aha" covert emotions which substantiate feelings of familiarity, then the combination of preserved familiarity processes in older adults (Prull et al., 2006) and declines in the ability to remember and reactivate false tags (Asp \& Tranel, 2013; Skurnik et al., 2005) may be responsible for the vulnerability of older adults to misleading information (Denburg et al., 2007), the failure of older adults to alter false beliefs following misinformation correction (Swire et al., 2017), and the increased propagation of misinformation on social media by older adults (Brashier \& Schacter, 2020; Grinberg et al., 2019).

This perspective would also indicate that the general illusory truth effect (Dechene et al., 2010; Hasher et al., 1977) results from increased "aha" emotions following memory searches which are measurable via EDA. Here, "aha" signals the recovery of information considered true 
that matches a given stimulus. Establishment of familiarity as distinct "aha" affective experiences may elucidate the familiarity-truth link (Begg et al., 1992) and inform how the epistemic emotion of certainty toward specific ideas is generated (Laukkonen, Webb, et al., 2018; Maglio \& Reich, 2019; Tormala \& Rucker, 2007). Indeed, artificially-induced "aha" experiences bias individuals to report false statements as true (Laukkonen et al., 2020). In the belief-default model, familiarity and "aha" may be regarded as functionally equivalent as this emotion signals the perceived successful recovery of information from memory without associated false tags. Whether retrieval processes recover propositions (inherent bearers of truth or falsity) or non-propositional information (Gawronski \& Strack, 2004; Mitchell et al., 2009), our proposed "aha" mechanism denotes two overlapping messages: 1) the information recovered is relevant to the current task (e.g., "old" responding during yes/no recognition memory tests), and 2) the information recovered is true/correct (i.e., it does not have false tags associated). Artificially-inducing feelings of familiarity with a low-amplitude buzz increases false memories (i.e., false alarms) on recognition memory tests (Goldinger \& Hansen, 2005). But more remarkably, artificially-inducing "aha" with anagrams strongly biases "old" judgments during recognition memory tests (i.e., the revelation effect, Abfalg \& Nadarevic, 2015; Frigo et al., 1999; Westerman \& Greene, 1996) and will induce false memories for personal autobiographical events and general semantic knowledge (Bernstein et al., 2004; Bernstein et al., 2002). Indeed, task difficulty (i.e., increased "impasses") moderates both the illusory truth effect and the revelation effect (Abfalg et al., 2017; Dechene et al., 2009). Conceptualizing familiarity as "aha" may address these diverse findings. Given the recent proliferation in misinformation (Lewandowsky et al., 2017), understanding the mechanisms of credulity to false information, the 
formation of intransigent incorrect beliefs (Lewandowsky et al., 2013), and optimal strategies for belief correction (Swire et al., 2017) are critical for future research to investigate.

One limitation of these studies is the lack of diversity in our participant samples. While our data do highlight important age-related effects drawn from developmental populations, the majority of the participants are "W.E.I.R.D." from a worldwide perspective (Henrich et al., 2010). Although we do not predict different results on the basis of ethnic, racial, gender, or social class variables, we do not explore these potential interactions in the work presented here. Given this lack of diversity, generalizability across samples is currently undetermined with empirical data.

EDA measurement during false memory paradigms (Loftus et al., 1978; Volz et al., 2017) may aid understanding the variables that promote "believed" false memories (e.g., Frenda et al., 2014; Riba et al., 2015; Roediger \& Geraci, 2007; Zhu et al., 2010). In the epistemic emotion hypothesis of the veridical EDA results, "believed" false memories should evoke similarly high EDA magnitudes (i.e., covert "aha" emotions) as "believed" true memories (Volz et al., 2017), and "guessed" false memories should evoke low EDA magnitudes relative to "believed" true memories ${ }^{29}$. Our results suggest "believed" and "guessed" false memory responses may be categorized on the basis of EDA magnitude.

The robust ordinal EDA magnitude results suggest epistemic emotion elicitation is endemic in true and false memory retrieval processes and that veridical identification is distinct from non-veridical, arbitrary source identification. Considering a proposition true or false may enhance memory recollection relative to considering the same proposition belonging to one of

\footnotetext{
29 "Guessed" responding to lures on the Deese-Roediger-McDermott paradigm (Roediger \& McDermott, 1995) may explain difference of true and false recognition in Baioui et al. (2012). See Volz et al. (2017) for contradictory data and a discussion.
} 
two arbitrary categories (Figure 6a, 6d, 7a, Supplementary Figure 5; Begg et al., 1992; Nadarevic \& Erdfelder, 2013). The evidence indicates that veridical assessment and categorization is not the mere association of a proposition with the arbitrary linguistic terms true and false, but rather represents a more basic assignment of ideas that will promote advantageous behavior in the environment (Asp \& Tranel, 2013; Gilbert, 1991). As primitive learning mechanisms initially evolved (Ginsburg \& Jablonka, 2010), animals gained the novel ability to adapt their behavior ontogenetically. Certainly, with this "quantal leap" (Ginsburg \& Jablonka, 2010) in terms of adaptability, an ability to "unlearn" when environmental contingencies changed following initial learning closely co-evolved (while keeping a record of the original learning). Thus, extinction learning mechanisms (Bouton, 2002, 2004; Rescorla, 2001) which are present in animals with simple nerve networks (e.g., Haralson et al., 1975; Hawkins et al., 2006; Rushforth, 1973) likely evolved with basic association learning to reduce behavior toward the initial learned association. Asp and Tranel's (2013) belief-default False Tagging Theory (FTT) asserts that extinction learning is the application of false tags to the original learning. Thus, the FTT conceives of human falsification to propositions as utilizing similar processes for extinction learning mechanisms to simple learned associations (e.g., Quirk et al., 2006). In this perspective, falsification is a fundamental process inhibiting actions toward disadvantageous outcomes (Asp \& Tranel, 2013; Porot \& Mandelbaum, 2020).

Taken together, the current research support belief-default perspectives indicating represented propositions are ballistic (Gilbert, 1993; Mandelbaum, 2014). Without an activation of an affixed false tag, a represented proposition will induce cognition-consistent action. Represented propositions that are contradictory with other represented propositions will elicit a false tag which may be affixed to proposition representations by a secondary process (Asp \& 
Tranel, 2013). Credulity, then, results from a failure to activate a false tag when encountering novel information contradictory to extant mental information considered true or remembering a previously encountered false propositions without activation of its initially encoded false tag. Future work should focus on identifying the myriad of variables that render individuals susceptible to believing false information.

\section{Acknowledgements}

Several key arguments of this article were invigorated by lectures from the Belief-Default Symposium held at Hamline University in the spring of 2018. We deeply thank all the attendees including: Dr. Daniel Gilbert, Dr. Edwin Curley, Dr. Jake Quilty-Dunn, Dr. Eric Mandelbaum, Dr. Justin Steinberg, Dr. Ruth Mayo, Dr. Uri Hasson, Dr. Maj-Britt Isberner, Dr. Lisa Fazio, Dr. Vikram Jaswal, Dr. Tali Sharot, Dr. Timothy Levine, Dr. Briony Swire, Dr. Joanne Miller, Dr. Christina Farhart, and Dr. Joshua Compton.

In addition, we thank Dr. Eric Mandelbaum for the ongoing, stimulating discussions regarding belief-default perspectives, Dr. Myrto Pantazi for help with the mixed-effects models analyzing true/false recognition memory test data, and the University of Iowa Libraries for their excellent support of research. A special thank you goes to Dr. Daniel Gilbert for his inspiring, seminal examinations of belief fixations models and his much-appreciated encouragement of the present article's first author.

\section{Funding}

This work was supported by the National Institute of Mental Health (1 P50 MH094258), the Kiwanis Foundation, an internal Hamline University Dean's Faculty Development Grant, a Psi Chi Undergraduate Research Grant, and a generous personal donation from Dr. Jerry Artz.

\section{Author Contributions}


E.A. designed the research; E.A., M.A, K.W., and E.P. collected data; E.A., L.K., A.J.

analyzed the data; and E.A. was the primary author of the manuscript with L.K., A.J., M.A.,

K.W., E.P., J.P, P.M., N.D., and D.T as secondary authors. All authors discussed the results and commented on the manuscript.

\section{References}

Abfalg, A., Currie, D., \& Bernstein, D. M. (2017). Task difficulty moderates the revelation effect. Memory \& Cognition, 45, 664-676.

Abfalg, A., \& Nadarevic, L. (2015). A word of warning: Instructions and feedback cannot prevent the revelation effect. Consciousness and Cognition, 34, 75-86.

Arango-Munoz, S. (2014). The nature of epistemic feelings. Philosophical Psychology, 27, 193-211.

Asp, E. W., Manzel, K., Koestner, B., Cole, C. A., Denburg, N. L., \& Tranel, D. (2012). A neuropsychological test of belief and doubt: Damage to ventromedial prefrontal cortex increases credulity for misleading advertising. Frontiers in Neuroscience, 6, 1-9.

Asp, E. W., \& Tranel, D. (2013). False Tagging Theory: Toward a unitary account of prefrontal cortex function. In D. T. Stuss \& R. T. Knight (Eds.), Principles of Frontal Lobe Function (2nd ed., pp. 383-416). New York: Oxford University Press.

Baioui, A., Ambach, W., Walter, B., \& Vaitl, D. (2012). Psychophysiology of false memories in a DeeseRoediger-McDermott paradigm with visual scences. PLOS ONE, 7, 1-9.

Batchelder, W. H., \& Batchelder, E. (2008). Metacognitive guessing strategies in source monitoring. In J. Dunlosky \& R. A. Bjork (Eds.), Handbook of metamemory and memory (pp. 211-244). New York: Psychology Press.

Batchelder, W. H., \& Riefer, D. M. (1990). Multinomial processing models of source monitoring. Psychological Review, 97, 548-564.

Bayen, U. J., Nakamura, G. V., Dupuis, S. E., \& Yang, C. (2000). The use of schematic knowledge about sources in source monitoring. Memory \& Cognition, 28, 480-500.

Bechara, A., \& Damasio, A. (2005). The somatic marker hypothesis: A neural theory of economic decision. Games and Economic Behavior, 52, 336-372.

Bechara, A., Damasio, H., Tranel, D., \& Damasio, A. R. (1997). Deciding advantageously before knowing the advantageous strategy. Science, 275, 1293-1295.

Begg, I. M., Anas, A., \& Farinacci, S. (1992). Dissociation of processes in belief: Source recollection, statement familiarity, and the illusion of truth. Journal of Experimental Psychology: General, $121,446-458$.

Ben-Shakhar, G. (1985). Standardization within individuals: Simple method to neutralize individual differences in skin conductance. Psychophysiology, 22, 292-299.

Bendana, J., \& Mandelbaum, E. (in press). The fragmentation of belief. In C. Kindermann, C. Borgoni, \& A. Onofri (Eds.), The fragmentation of mind. Oxford: Oxford University Press.

Bernstein, D. M., Godfrey, R. D., Davison, A., \& Loftus, E. F. (2004). Conditions affecting the revelation effect for autobiographical memory. Memory \& Cognition, 32, 455-462.

Bernstein, D. M., Whittlesea, B. W. A., \& Loftus, E. F. (2002). Increasing confidence in remote autobiographical memory and general knowledge: Extensions of the revelation effect. Memory \& Cognition, 2002, 432-438.

Bouton, M. E. (2002). Context, ambiguity, and unlearning: Sources of relapse after behvaioral extinction. Biological Psychiatry, 52, 976-986. 
Bouton, M. E. (2004). Context and behavioral processes in extinction. Learning \& Memory, 11, 485-494. Brainerd, C. J., Stein, L. M., Silveira, R. A., Rohenkohl, G., \& Reyna, V. F. (2008). How does negative emotion cause false memories? Psychological Science, 19, 919-925.

Brainerd, C. J., Wright, R., Reyna, V. F., \& Mojardin, A. H. (2001). Conjoint recognition and phantom recollection. Journal of Experimental Psychology: Learning, Memory, and Cognition, 27, 307327.

Brashier, N. M., \& Schacter, D. L. (2020). Aging in an era of fake news. Current Directions in Psychological Science, 29, 316-323.

Brown, R., James, C., Henderson, L. A., \& Macefield, V. G. (2012). Autonomic markers of emotional processing: Skin sympathetic nerve activity in humans during exposure to emotionally charged images. Frontiers in Physiology, 3, 1-6.

Carpenter, P. A., \& Just, M. A. (1975). Sentence comprehension: A psycholinguistic processing model of verification. Psychological Review, 82, 45-73.

Chen, Y. W. (2002). Unwanted beliefs: Age differences in beliefs of false information. Aging, Neuropsychology, and Cognition, 9, 217-230.

Chen, Y. W., \& Blanchard-Fields, F. (2000). Unwanted thought: Age differences in the correction of social judgments. Psychology and Aging, 15, 475-482.

Craik, F. (1982). Selective changes in encoding as a function of reduced processing capacity. In F. Klix, J. Hoffmann, \& E. van der Meer (Eds.), Cognitive Research in Psychology (pp. 152-161). Berlin: Deutscher Verlag der Wissenschaften.

Critchley, H. (2002). Electrodermal responses: What happens in the brain. The Neuroscientist, 8, 132142.

Damasio, A. (1994). Decartes' Error: Emotion, Reason and the Human Brain. New York: Grosset/Putnam.

Damasio, A., \& Carvalho, G. B. (2013). The nature of feelings: Evolutionary and neurobiological origins. Nature Reviews Neuroscience, 14, 143-152.

Dawson, M. E., Schell, A. M., \& Filion, D. L. (2000). The electrodermal system. In J. T. Cacioppo, L. G. Tassinary, \& G. G. Berntson (Eds.), Handbook of psychophysiology (2nd ed., pp. 200-223). Cambridge, UK: Cambridge University Press.

Dechene, A., Stahl, C., Hansen, J., \& Wanke, M. (2009). Mix me a list: Context moderates the truth effect and the mere-exposure effect. Journal of Experimental Social Psychology, 45, 1117-1122.

Dechene, A., Stahl, C., Hansen, J., \& Wanke, M. (2010). The truth about the truth: A meta-analytic review of the truth effect. Personality and Social Psychology Review, 14, 238-257.

Denburg, N. L., Cole, C. A., Hernandez, M., Yamada, T. H., Tranel, D., Bechara, A., \& Wallace, R. B. (2007). The orbitofrontal cortex, real-world decision making, and normal aging. Annals of the New York Academy of Sciences, 1121, 480-498.

Duke, D., Fiacconi, C. M., \& Kohler, S. (2014). Parallel effects of processing fluency and positive affect on familiarity-based recognition decisions for faces. Frontiers in Psychology, 5, 1-11.

Egan, A. (2008). Seeing and believing: Perception, belief formation and the divided mind. Philosophical Studies, 140, 47-63.

Elkin, R. A., \& Leippe, M. R. (1986). Physiological arousal, dissonance, and attitude change: Evidence for a dissonance-arousal link and a "don't remind me" effect. Journal of Personality and Social Psychology, 51, 55-65.

Erdfelder, E., Auer, T., Hilbig, B., Abfalg, A., Moshagen, M., \& Nadarevic, L. (2009). Multinomial processing tree models: A review of the literature. Journal of Psychology, 217, 108-124.

Faul, F., Erdfelder, E., Lang, A. G., \& Buchner, A. (2007). G* Power 3: A flexible statistical power analysis program for the social, behavioral, and biomedical sciences. Behavior Research Methods, 39, 175-191.

Fazio, L. K. (2020). Repetition increases perceived truth even for known falsehoods. Collabra: Psychology, 6, 1-7. 
Fazio, L. K., Brashier, N. M., Payne, B. K., \& Marsh, E. J. (2015). Knowledge does not protect against illusory truth. Journal of Experimental Psychology: General, 144, 993-1002.

Fiacconi, C. M., Kouptsova, J. E., \& Kohler, S. (2017). A role for visceral feedback and interoception in feelings-of-knowing. Consciousness and Cognition, 53, 70-80.

Forgas, J. P. (2019). Happy believers and sad skeptics? Affective influences on gullibility. Current Directions in Psychological Science, 28, 306-313.

Frenda, S. J., Patihis, L., Loftus, E., Lewis, H. C., \& Fenn, K. M. (2014). Sleep deprivation and false memories. Psychological Science, 25, 1674-1681.

Frigo, L. C., Reas, D. L., \& Lecompte, D. C. (1999). Revelation without presentation: Counterfeit study list yields robust revelation effect. Memory \& Cognition, 27, 339-343.

Garcia-Marques, T., Mackie, D. M., Claypool, H. M., \& Garcia-Marques, L. (2004). Positivity can cue familiarity. Personality and Social Psychology Bulletin, 30, 585-593.

Garcia-Marques, T., Silva, R. R., \& Mello, J. (2016). Judging the truth-value of a statement in and out of a deep processing context. Social Cognition, 34, 40-54.

Garcia-Marques, T., Silva, R. R., Reber, R., \& Unkelbach, C. (2015). Hearing a statement now and believing the opposite later. Journal of Experimental Social Psychology, 56, 126-129.

Gawronski, B., \& Strack, F. (2004). On the propositional nature of cognitive consistency: Dissonance changes explicit, but not implicit attitudes. Journal of Experimental Social Psychology, 40, 535542.

Gilbert, D. T. (1991). How mental systems believe. American Psychologist, 46, 107-119.

Gilbert, D. T. (1993). The assent of man: Mental representation and the control of belief. In D. M. Wegner \& J. W. Pennebaker (Eds.), Handbook of mental control (pp. 57-87). Engelwood Cliffs, NJ: Prentice Hall.

Gilbert, D. T., Krull, D. S., \& Malone, P. S. (1990). Unbelieving the unbelievable: Some problems in the rejection of false information. Journal of Personality and Social Psychology, 59, 601-613.

Gilbert, D. T., Tafarodi, R. W., \& Malone, P. S. (1993). You can't not believe everything you read. Journal of Personality and Social Psychology, 65, 221-233.

Ginsburg, S., \& Jablonka, E. (2010). The evolution of associative learning: A factor in the Cambrian explosion. Journal of Theoretical Biology, 266, 11-20.

Goldinger, S. D., \& Hansen, W. A. (2005). Remembering by the seat of your pants. Psychological Science, 16, 525-529.

Gough, P. B. (1965). Grammatical transformations and speed of understanding. Journal of Verbal Learning and Verbal Behavior, 4, 107-111.

Grice, H. P. (1975). Logic and conversation. In P. Cole \& J. L. Morgan (Eds.), Syntax and Semantics: Vol 3. Speech Acts (pp. 41-58). New York: Seminar Press.

Grinberg, N., Joseph, K., Friedland, L., Swire-Thompson, B., \& Lazer, D. (2019). Fake news on Twitter during the 2016 U.S. presidential election. Science, 363, 374-378.

Haralson, J. V., Groff, C. I., \& Haralson, S. J. (1975). Classical conditioning in the sea anemone, Cribrina xanthogrammica. Physiology \& Behavior, 15, 455-460.

Harmon-Jones, E. (2000). Cognitive dissonance and experienced negative affect: Evidence that dissonance increases experienced negative affect even in the absence of aversive consequences. Personality and Social Psychology Bulletin, 26, 1490-1501.

Harris, S., Sheth, S. A., \& Cohen, M. S. (2008). Functional neuroimaging of belief, disbelief, and uncertainty. Annals of Neurology, 63, 141-147.

Hasher, L., Goldstein, D., \& Toppino, T. (1977). Frequency and the conference of referential validity. Journal of Verbal Learning and Verbal Behavior, 16, 107-112.

Hasson, U., Simmons, J. P., \& Todorov, A. (2005). Believe it or not. Psychological Science, 16, 566-571.

Hawkins, R. D., Clark, G. A., \& Kandel, E. R. (2006). Operant conditioning of gill withdrawal in Aplysia. The Journal of Neuroscience, 26, 2443-2448.

Henrich, J., Heine, S. J., \& Norenzayan, A. (2010). The weirdest people in the world? Behavioral and Brain Sciences, 33, 61-83. 
James, W. (1905). The Principles of Psychology (Vol. 2). New York: Henry Holt and Company. Judd, C. M., Westfall, J., \& Kenny, D. A. (2012). Treating stimuli as a random factor in social psychology: A new and comprehensive solution to a pervasive but largely ignored problem. Journal of Personality and Social Psychology, 103, 54-69.

Koch, A. S., \& Forgas, J. P. (2012). Feeling good and feeling truth: The interactive effects of mood and processing fluency on truth judgments. Journal of Experimental Social Psychology, 48, 481-485.

Kuhlman, B. G., \& Bayen, U. J. (2016). Metacognitive aspects of source monitoring. In J. Dunlosky \& S. Tauber (Eds.), The Oxford handbook of metamemory (pp. 149-168). New York: Oxford University Press.

Laukkonen, R. E., Ingledew, D. J., Kaveladze, B. T., Schooler, J. W., \& Tangen, J. M. (2018). The phenomenology of truth: The 'Aha!' experience as a heuritic in contexts of uncertainty. PsyArXiv, 1-47. doi:https://psyarxiv.com/9w56m/

Laukkonen, R. E., Kaveladze, B. T., Tangen, J. M., \& Schooler, J. W. (2020). The dark side of Eureka: Artificially induced Aha moments make facts feel true. Cognition, 196, 1-6.

Laukkonen, R. E., Webb, M., Salvi, C., Tangen, J., \& Schooler, J. (2018). Eureka heuristics: How feelings of insight signal the quality of a new idea. PsyArXiv, 1-30. doi:https://psyarxiv.com/ez3tn/

Lazer, D., Baum, M., Benkler, Y., Berinsky, A., Greenhill, K., Menczer, F., . . Zittrain, J. (2018). The science of fake news. Science, 359, 1094-1096.

Lee, E., \& Shin, S. (2019). Mediated misinformation: Questions answered, more questions to ask. American Behavioral Scientist, 1-18.

Lewandowsky, S., Ecker, U., \& Cook, J. (2017). Beyond misinformation: Understanding and coping with the "post-truth" era. Journal of Applied Research in Memory and Cognition, 6, 353-369.

Lewandowsky, S., Oberauer, K., \& Gignac, G. E. (2013). NASA faked the moon landing--therefore, (climate) science is a hoax: An anatomy of the motivated rejection of science. Psychological Science, 24, 622-633.

Leys, C., Ley, C., Klein, O., Bernard, P., \& Licata, L. (2013). Detecting outliers: Do not use standard deviation around the mean, use absolute deviation around the median. Journal of Experimental Social Psychology, 49, 764-766.

Loftus, E. F., Miller, D. G., \& Burns, H. J. (1978). Semantic integration of verbal information into a visual memory. Journal of Experimental Psychology: Human Learning and Memory, 4, 19-31.

Maglio, S. J., \& Reich, T. (2019). Feeling certain: Gut choice, the true self, and attitude certainty. Emotion, 19, 876-888.

Mandelbaum, E. (2014). Thinking is believing. Inquiry, 57, 55-96.

Meiser, T., \& Broder, A. (2002). Memory for multidimensional source information. Journal of Experimental Psychology: Learning, Memory, and Cognition, 28, 116-137.

Mercier, H. (2017). How gullible are we? A review of the evidence from psychology and social science. Review of General Psychology, 21, 103-122.

Mitchell, C. J., De Houwer, J., \& Lovibond, P. F. (2009). The propositional nature of human associative learning. Behavioral and Brain Sciences, 2009, 183-246.

Morris, A. L., Cleary, A. M., \& Still, M. L. (2008). The role of autonomic arousal in feelings of familiarity. Consciousness and Cognition, 17, 1378-1385.

Moshagen, M. (2010). multiTree: A computer program for the analysis of multinomal processing tree models. Behavior Research Methods, 42, 42-54.

Nadarevic, L., \& Erdfelder, E. (2013). Spinoza's error: Memory for truth and falsity. Memory \& Cognition, 41, 176-186.

Nadarevic, L., \& Erdfelder, E. (2019). More evidence against the Spinozan model: Cognitive load diminishes memory for "true" feedback. Memory \& Cognition, 1-15.

Pantazi, M., Kissine, M., \& Klein, O. (2018). The power of the truth bias: False information affects memory and judgment even in the absence of distraction. Social Cognition, 36, 167-198. 
Pennycook, G., Cheyne, J. A., Koehler, D. J., \& Fugelsang, J. A. (2013). Belief bias during reasoning among religious believers and skeptics. Psychonomic Bulletin \& Review, 20, 806-811.

Pennycook, G., \& Rand, D. G. (2019). Who falls for fake news? The roles of bullshit receptivity, overclaiming, familiarity, and analytic thinking. Journal of Personality, 1-16.

Pennycook, G., Tranel, D., Warner, K., \& Asp, E. W. (2019). Beyond reasonable doubt: Cognitive and neuropsychological implications for religious disbelief. In A. Coles \& J. Collicutt (Eds.), Neurology and Religion (pp. 115-129). Cambridge: Cambridge University Press.

Plouffe, L., \& Stelmack, R. (1984). The electrodermal orienting response and memory: An analysis of age differences in picture recall. Psychophysiology, 21, 191-198.

Porot, N., \& Mandelbaum, E. (2020). The science of belief: A progess report. WIREs Cognitive Science, $1-17$.

Prull, M. W., Dawes, L. L., Martin, A. M., Rosenberg, H. F., \& Light, L. L. (2006). Recollection and familiarity in recognition memory: Adult age differences and neuropsychological test correlates. Psychology and Aging, 21, 107-118.

Quene, H., \& van den Bergh, H. (2008). Examples of mixed-effects modeling with crossed random effects and with binomial data. Journal of Memory and Language, 59, 413-425.

Quirk, G. J., Garcia, R., \& Gonzalez-Lima, F. (2006). Prefrontal mechanisms in extinction of conditioned fear. Biological Psychiatry, 60, 337-343.

Rescorla, R. A. (2001). Experimental extinction. In R. R. Mowrer \& S. B. Klein (Eds.), Handbook of Contemporary Learning Theories (pp. 119-154). Mahwah, NJ: Erlbaum.

Riba, J., Valle, M., Sampedro, F., Rodriquez-Pujadas, A., Martinez-Horta, S., Kulisevsky, J., \& Rodriquez-Fornells, A. (2015). Telling true from false: Cannabis users show increased susceptibility to false memories. Molecular Psychiatry, 20, 772-777.

Richter, T., Schroeder, S., \& Wöhrmann, B. (2009). You don't have to believe everything you read: Background knowledge permits fast and efficient validation of information. Journal of Personality and Social Psychology, 96, 538-558.

Riefer, D. M., \& Batchelder, W. H. (1988). Multinomial modeling and the measurement of cognitive processes. Psychological Review, 95, 318-339.

Riefer, D. M., Hu, X., \& Batchelder, W. H. (1994). Response strategies in source monitoring. Journal of Experimental Psychology: Learning, Memory, and Cognition, 20, 680-693.

Rips, L. J., \& Marcus, S. L. (1977). Suppositions and the analysis of conditional sentences. In M. A. Just \& P. A. Carpenter (Eds.), Cognitive processes in comprehension (pp. 185-220). Hillsdale, NJ: Erlbaum.

Roediger, H. L., \& Geraci, L. (2007). Aging and the misinformation effect: A neuropsychological analysis. Journal of Experimental Psychology: Learning, Memory, and Cognition, 33, 321-334.

Roediger, H. L., \& McDermott, K. B. (1995). Creating false memories: Remembering words not presented in lists. Journal of Experimental Psychology: Learning, Memory, and Cognition, 21, 803-814.

Rushforth, N. B. (1973). Behavioral modifications in coelenterates. In W. C. Corning, J. A. Dyal, \& A. O. D. Willows (Eds.), Invertebrate learning (pp. 123-169). New York: Plenum Press.

Sa, W. C., West, R. F., \& Stanovich, K. E. (1999). The domain specificity and generality of belief bias: Searching for a generalizable critical thinking skill. Journal of Educational Psychology, 91, 497510.

Salvi, C., Bricolo, E., Kounios, J., Bowden, E., \& Beeman, M. (2016). Insight solutions are correct more often than analytic solutions. Thinking \& Reasoning, 4, 443-460.

Schwarz, N. (2012). Feelings-as-information-theory. In P. Van Lange, A. Kruglanski, \& E. T. Higgins (Eds.), Handbook of theories of social psychology. Thousands Oaks, CA: Sage.

Shearer, D., \& Mikulka, P. (1996). Effect of facial familiarity and task requirement on electrodermal activity. American Journal of Psychology, 109, 131-137. 
Shen, W., Tong, Y., Yuan, Y., Zhan, H., Liu, C., Luo, J., \& Cai, H. (2018). Feeling the insight: Uncovering somatic markers of the "aha" experience. Applied Psychophysiological Biofeedback, $43,13-21$.

Siddle, D. (1991). Orienting, habituation, and resource allocation: An associative analysis. Psychophysiology, 28, 245-259.

Silva, R. R., Garcia-Marques, T., \& Mello, J. (2016). The differential effects of fluency due to repetition and fluency to color contrast on judgments of truth. Psychological Research, 80, 821-837.

Skurnik, I., Yoon, C., Park, D. C., \& Schwarz, N. (2005). How warnings about false claims become recommendations. Journal of Consumer Research, 31, 713-724.

Sperber, D., Clement, F., Heintz, C., Mascaro, O., Mercier, H., Origgi, G., \& Wilson, D. (2010). Epistemic vigilance. Mind \& Language, 25, 359-393.

Spinoza, B. (1982). The ethics and selected letters (S. Shirley, Trans.). Indianapolis, IN: Hackett.

Staddon, J. E. R., King, M., \& Lockhead, G. R. (1980). On sequential effects in absolute judgment experiments. Journal of Experimental Psychology: Human Perception and Performance, 6, 290301.

Stanovich, K. E. (2003). The fundamental computational biases of human cognition: Heuristics that (sometimes) impair decision making and problem solving. In J. E. Davidson \& R. J. Sternberg (Eds.), Psychology of problem solving (pp. 291-342). New York: Cambridge University Press.

Stelmack, R., Plouffe, L., \& Winogron, H. W. (1983). Recognition memory and the orienting response: An analysis of the encoding of pictures and words. Biological Psychology, 16, 49-63.

Street, C. N. H., \& Kingstone, A. (2017). Aligning Spinoza with Descartes: An informed Cartesian account of the truth bias. British Journal of Psychology, 108, 453-466.

Street, C. N. H., \& Richardson, D. C. (2015). Descartes versus Spinoza: Truth, uncertainty, and bias. Social Cognition, 33, 227-239.

Swire, B., Ecker, U., \& Lewandowsky, S. (2017). The role of familiarity in correcting inaccurate information. Journal of Experimental Psychology: Learning, Memory, and Cognition, 12, 19481961.

Tormala, Z. L., \& Rucker, D. D. (2007). Attitude certainty: A review of past findings and emerging perspectives. Social and Personality Psychology Compass, 1, 469-492.

Tranel, D., Fowles, D. C., \& Damasio, A. (1985). Electrodermal discrimination of familiar and unfamiliar faces: A methodology. Psychophysiology, 22, 403-408.

Unkelbach, C. (2007). Reversing the truth effect: Learning the interpretation of processing fluency in judgments of truth. Journal of Experimental Psychology: Learning, Memory, and Cognition, 33, 219-230.

Unkelbach, C., \& Rom, S. C. (2017). A referential theory of the repetition-induced truth effect. Cognition, $160,110-126$.

Unkelbach, C., \& Stahl, C. (2009). A multinomial modeling approach to dissociate different componets of the truth effect. Consciousness and Cognition, 18, 22-38.

Volz, K., Leonhart, R., Stark, R., Vaitl, D., \& Ambach, W. (2017). Psychophysiological correlates of the misinformation effect. International Journal of Psychophysiology, 117, 1-9.

Vosoughi, S., Roy, D., \& Aral, S. (2018). The spread of true and false news online. Science, 359, 11461151.

Webb, M. E., Little, D. R., \& Cropper, S. J. (2018). Once more with feeling: Normative data for the aha experience in insight and noninsight problems. Behavioral Research Methods, 50, 2035-2056.

Weisberg, R. W. (2015). Toward an integrated theory of insight in problem solving. Thinking \& Reasoning, 21, 5-39.

Westerman, D. L., \& Greene, R. L. (1996). On the generality of the revelation effect. Journal of Experimental Psychology: Learning, Memory, and Cognition, 22, 1147-1153.

Wiegersma, S. (1982). Can repetition avodiance in randomization be explained by randomness concepts? Psychological Research, 44, 189-198.

Wittgenstein, L. (1969). On certainty. Oxford: Blackwell. 
Zhu, B., Chen, C., Loftus, E., Lin, C., He, Q., Chen, C., . . Dong, Q. (2010). Individual differences in false memory from misinformation: Cognitive factors. Memory, 18, 543-555. 
Table 1

Possible response outcomes during traditional true/false recognition memory tests

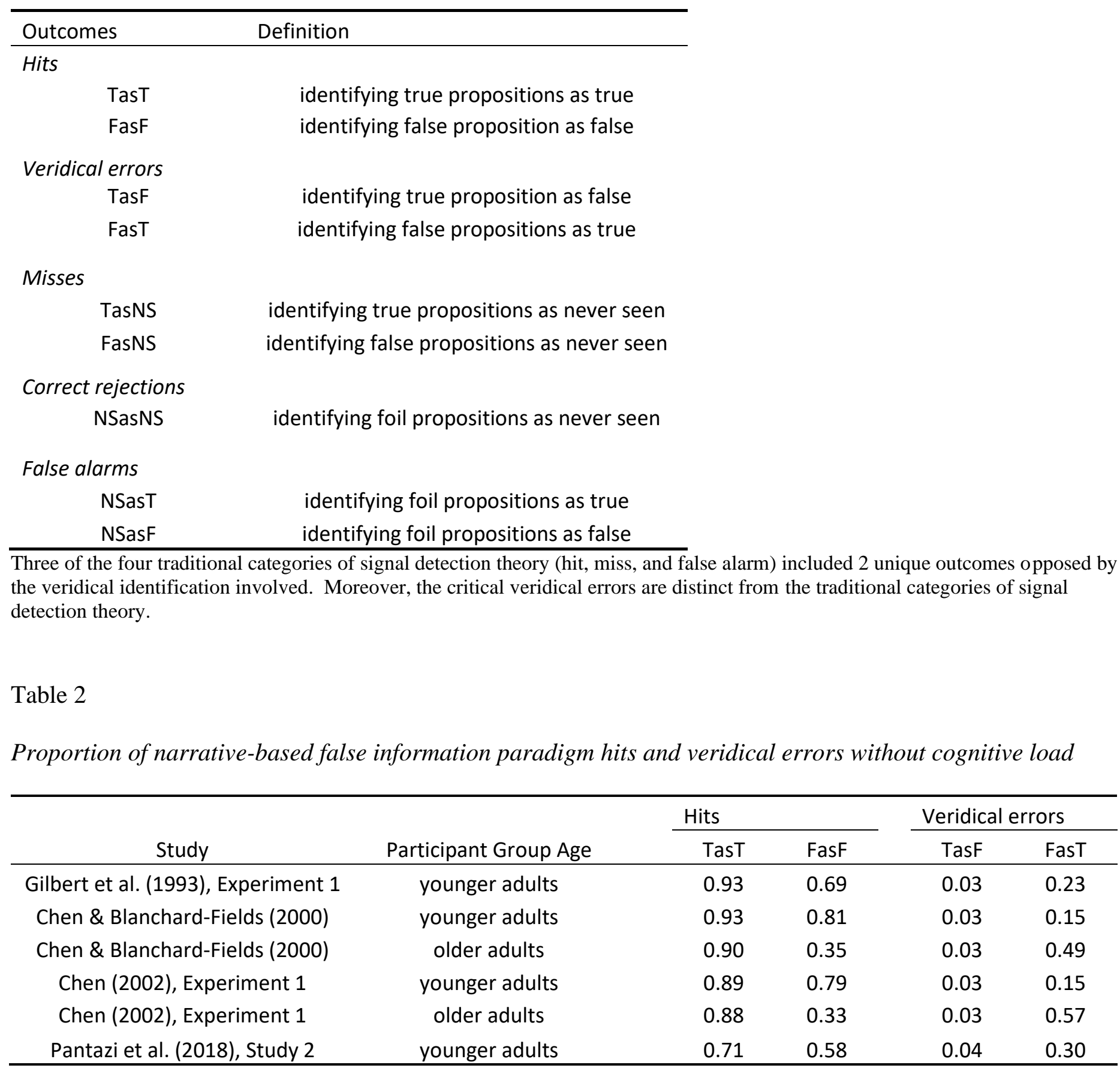

Narrative-based false information paradigms have unequal outcomes within their hits (TasT > FasF) and veridical errors (FasT > TasF). 
Table 3

DJT belief-default MPT model parameter estimates for Studies 1-3

\begin{tabular}{|c|c|c|c|c|c|c|c|c|}
\hline \multirow[b]{2}{*}{ Type } & \multicolumn{2}{|l|}{ Study 1 (YA) } & \multicolumn{2}{|l|}{ Study 2 (YA) } & \multicolumn{2}{|l|}{ Study 3 (YA) } & \multicolumn{2}{|l|}{ Study 3 (OA) } \\
\hline & $\begin{array}{l}\text { Parameter } \\
\text { estimate (SE) }\end{array}$ & $95 \% \mathrm{Cl}$ & $\begin{array}{l}\text { Parameter } \\
\text { estimate (SE) }\end{array}$ & $95 \% \mathrm{Cl}$ & $\begin{array}{l}\text { Parameter } \\
\text { estimate (SE) }\end{array}$ & $95 \% \mathrm{Cl}$ & $\begin{array}{l}\text { Parameter } \\
\text { estimate (SE) }\end{array}$ & $95 \% \mathrm{Cl}$ \\
\hline \multicolumn{9}{|l|}{ Memory } \\
\hline$D_{\text {true }}$ & $.89(.01)$ & {$[.87, .92]$} & $.87(.01)$ & {$[.84, .89]$} & $.90(.01)$ & {$[.88, .93]$} & $.85(.02)$ & {$[.82, .89]$} \\
\hline$D_{\text {false }}$ & $.89(.02)$ & {$[.86, .94]$} & $.88(.02)$ & $\begin{array}{c}{[.85, .91]} \\
{[.86}\end{array}$ & $.89(.02)$ & {$[.86, .93]$} & $.79(.03)$ & {$[.72, .85]$} \\
\hline$D_{N S}$ & $.90(.05)$ & {$[.81, .99]$} & $.93(.04)$ & 1.00] & $.94(.03)$ & {$[.88,1.00]$} & $.92(.04)$ & {$[.84, .99]$} \\
\hline$d_{\text {false }}$ & $.92(.01)$ & {$[.89, .95]$} & $.91(.01)$ & {$[.89, .94]$} & $.91(.01)$ & {$[.89, .94]$} & $.66(.02)$ & {$[.62, .71]$} \\
\hline$Z \emptyset, Z \sim t$ & $.03(.01)$ & {$[0, .05]$} & $.05(.01)$ & {$[.03, .08]$} & $.02(.01)$ & {$[0, .04]$} & $.07(.02)$ & {$[.04, .10]$} \\
\hline$Z_{\neq t}$ & $.34(.03)$ & {$[.28, .39]$} & $.19(.02)$ & {$[.16, .23]$} & $.23(.02)$ & {$[.18, .27]$} & $.33(.03)$ & {$[.27, .38]$} \\
\hline$z_{\sim f}$ & $.18(.03)$ & {$[.12, .24]$} & $.14(.02)$ & {$[.09, .19]$} & $.14(.03)$ & {$[.09, .19]$} & $.19(.03)$ & {$[.12, .25]$} \\
\hline$n$ & $.96(.02)$ & {$[.92, .99]$} & $.96(.01)$ & {$[.93, .98]$} & $.95(.01)$ & {$[.92, .97]$} & $.94(.02)$ & {$[.90, .97]$} \\
\hline$S_{\text {true }}$ & $.47(.27)$ & {$[0,1.00]$} & $.64(.25)$ & $\begin{array}{l}{[.14} \\
1.00]\end{array}$ & $.88(.21)$ & {$[.47,1.00]$} & $.33(.23)$ & {$[0, .79]$} \\
\hline$s_{\text {false }}$ & $.37(.42)$ & {$[0,1.00]$} & $.43(.29)$ & {$[0,1.00]$} & $.27(.30)$ & {$[0, .85]$} & $.07(.41)$ & {$[0, .89]$} \\
\hline \multicolumn{9}{|l|}{ Guessing } \\
\hline$b$ & $.36(.08)$ & {$[.21, .51]$} & $.28(.06)$ & {$[.17, .39]$} & $.36(.07)$ & {$[.22, .50]$} & $.43(.07)$ & {$[.30, .56]$} \\
\hline$g_{\text {true }}$ & .50 (constant) & NA & .50 (constant) & NA & .50 (constant) & NA & .50 (constant) & NA \\
\hline
\end{tabular}

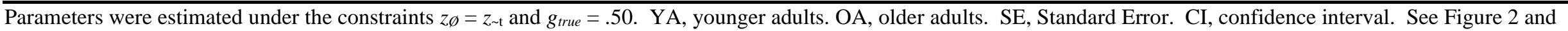

Study 1's statistical method description for individual parameter explanations. Older adults showed reliably lower $d_{f a l s e}$ than younger adults (Study 3 Results). 
Table 4

HLT Cartesian MPT model parameter estimates for Study 3

\begin{tabular}{|c|c|c|c|c|}
\hline \multirow[b]{2}{*}{ Type } & \multicolumn{2}{|l|}{ Study 3 (YA) } & \multicolumn{2}{|l|}{ Study 3 (OA) } \\
\hline & Parameter estimate (SE) & $95 \% \mathrm{Cl}$ & Parameter estimate (SE) & $95 \% \mathrm{Cl}$ \\
\hline \multicolumn{5}{|l|}{ Memory } \\
\hline$D_{\text {true }} D_{\text {false }}$ & $.96(.01)$ & {$[.95, .98]$} & $.96(.01)$ & {$[.94, .98]$} \\
\hline$d_{\text {true }}$ & $.47(.05)$ & {$[.37, .57]$} & $.36(.07)$ & {$[.21, .51]$} \\
\hline$d_{\text {false }}$ & $.48(.05)$ & {$[.39, .58]$} & $.28(.08)$ & {$[.12, .43]$} \\
\hline \multicolumn{5}{|l|}{ Guessing } \\
\hline$b$ & $.04(.02)$ & {$[.01, .07]$} & $.06(.03)$ & {$[0, .12]$} \\
\hline$a_{\text {true, }} g_{\text {true }}$ & .50 (constant) & NA & .50 (constant) & NA \\
\hline
\end{tabular}

Parameters were estimated under the constraints $D_{\text {true }}=D_{\text {false }}$ and $a_{\text {true }}=g_{\text {true }}=.50$. YA, younger adults. OA, older adults. SE, Standard Error. CI, confidence interval. See Figure 5 and Study 3's statistical method description for individual parameter explanations. Older adults showed reliably lower $d_{\text {false }}$ than younger adults (Study 3 Results). 
Figure 1

Stages and possible propositional states of Cartesian, Spinozan, and False Tagging Theory belief-fixation models

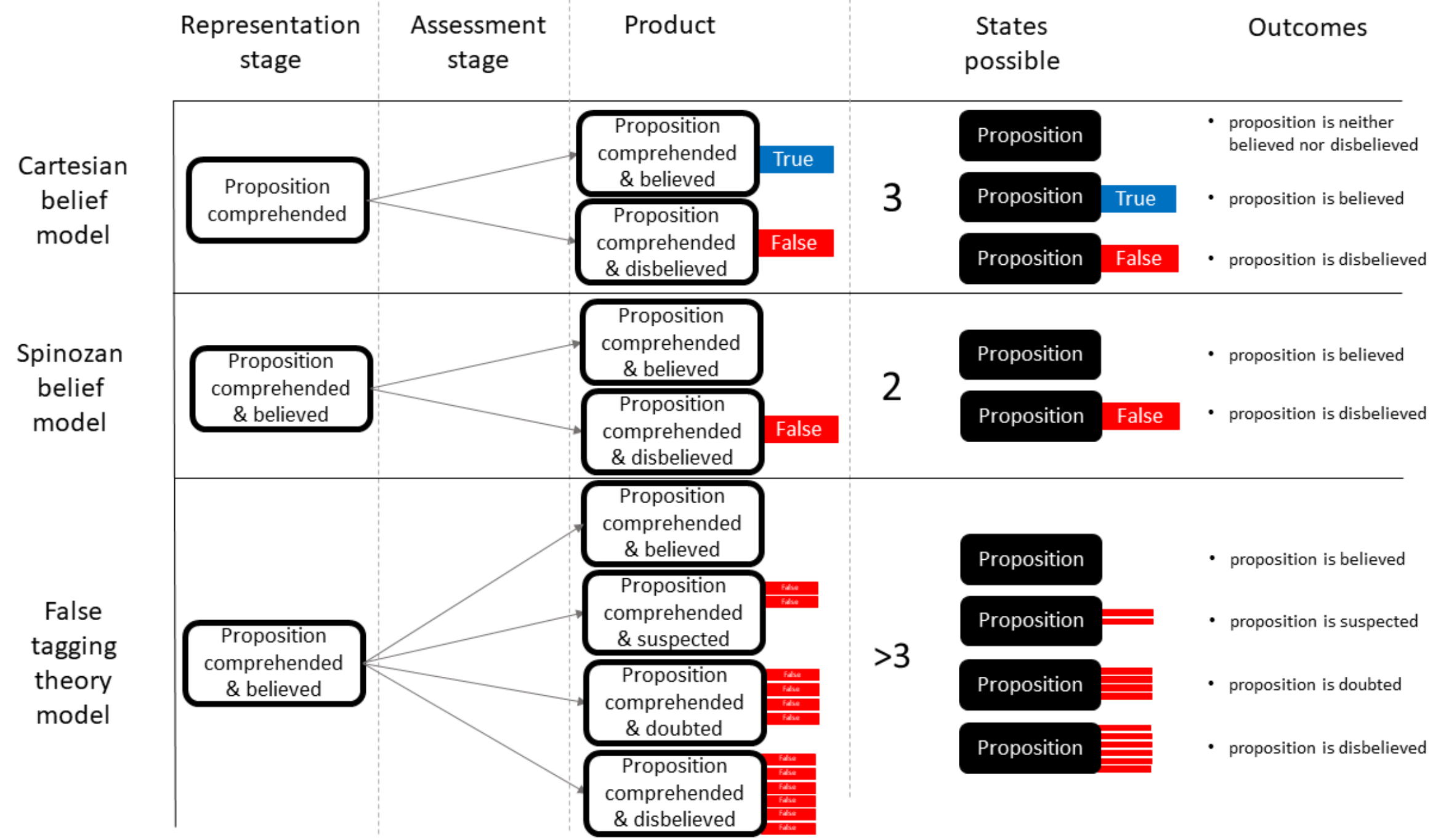

Three states are possible in the Cartesian belief model, including a merely represented proposition without a true or false tag associated. Belief-default models (Spinozan, False Tagging Theory) assert that propositions without affixed false tags are believed. The False Tagging Theory delineates a continuous false tagging process enabling degrees of belief and a graded inhibition toward a proposition. 
Figure 2

\section{Structure and parameters of the DJT MPT models}

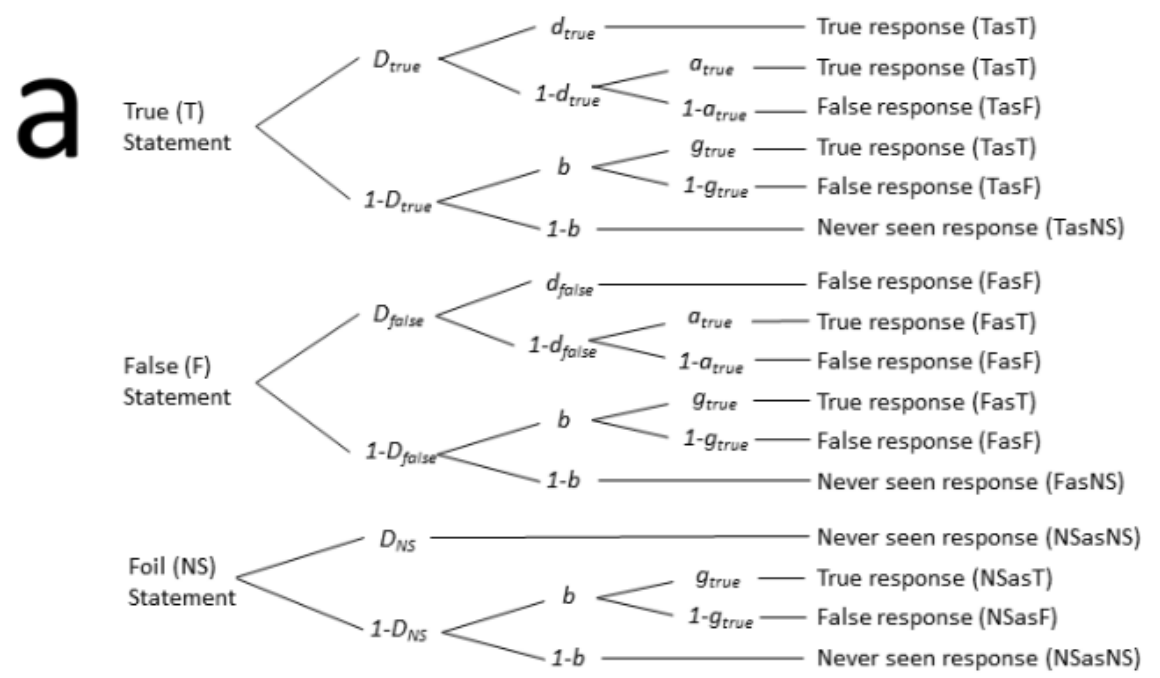

b

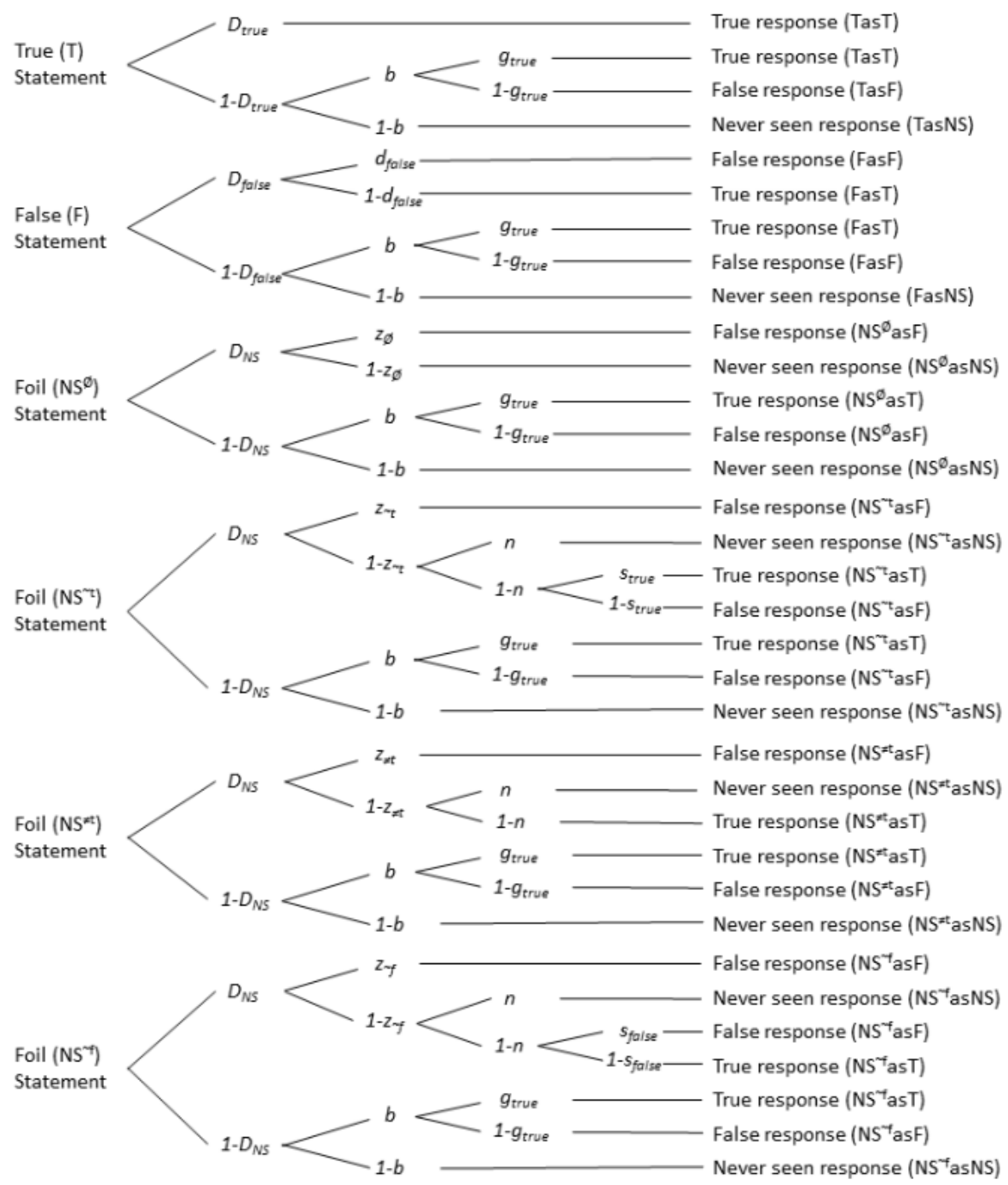


Each model consists of separate processing trees for true, false, and foil statements. Tree branches represent a possible sequence of cognitive processes resulting in a true, false, or never seen response. Model parameters reflect transition probabilities from left to right. a) DJT Cartesian MPT model: $D=$ probability of statement recognition or foil detection, $d=$ probability of correctly

discriminating the source (i.e., in this case true or false), $b=$ probability of guessing "old" to items not recognized nor identified as a foil, $g=$ probability of guessing that a non-detected item belongs to a particular source, and $a=$ probability of guessing that a detected but non-discriminated item belongs to a particular source. b) DJT belief-default MPT model. Additional parameters include: $z=$ probability that a foil statement related to prior knowledge is misidentified as false, $n=$ probability that a foil statement related to prior knowledge not misidentified as false is correctly identified as never seen, and $s=$ probability that evaluation of the familiarity of a related foil induces a false alarm. NS ${ }^{\varnothing}$, foil statements not related to true nor false statements; $\mathrm{NS}^{\sim \mathrm{t}}$, foil statements similar to true statements but not mutually exclusive with them; $\mathrm{NS}^{\neq t}$, foil statements similar to true statements and mutually exclusive with them; $\mathrm{NS}^{\sim \mathrm{f}}$, foil statements similar to false statements. Of note, the DJT belief-default MPT model does not contain a $d_{\text {true }}$ parameter. DJT data from Studies 1-3 fit the belief-default MPT model well and fit the Cartesian MPT model poorly. 
Figure 3

Study 1 DJT results
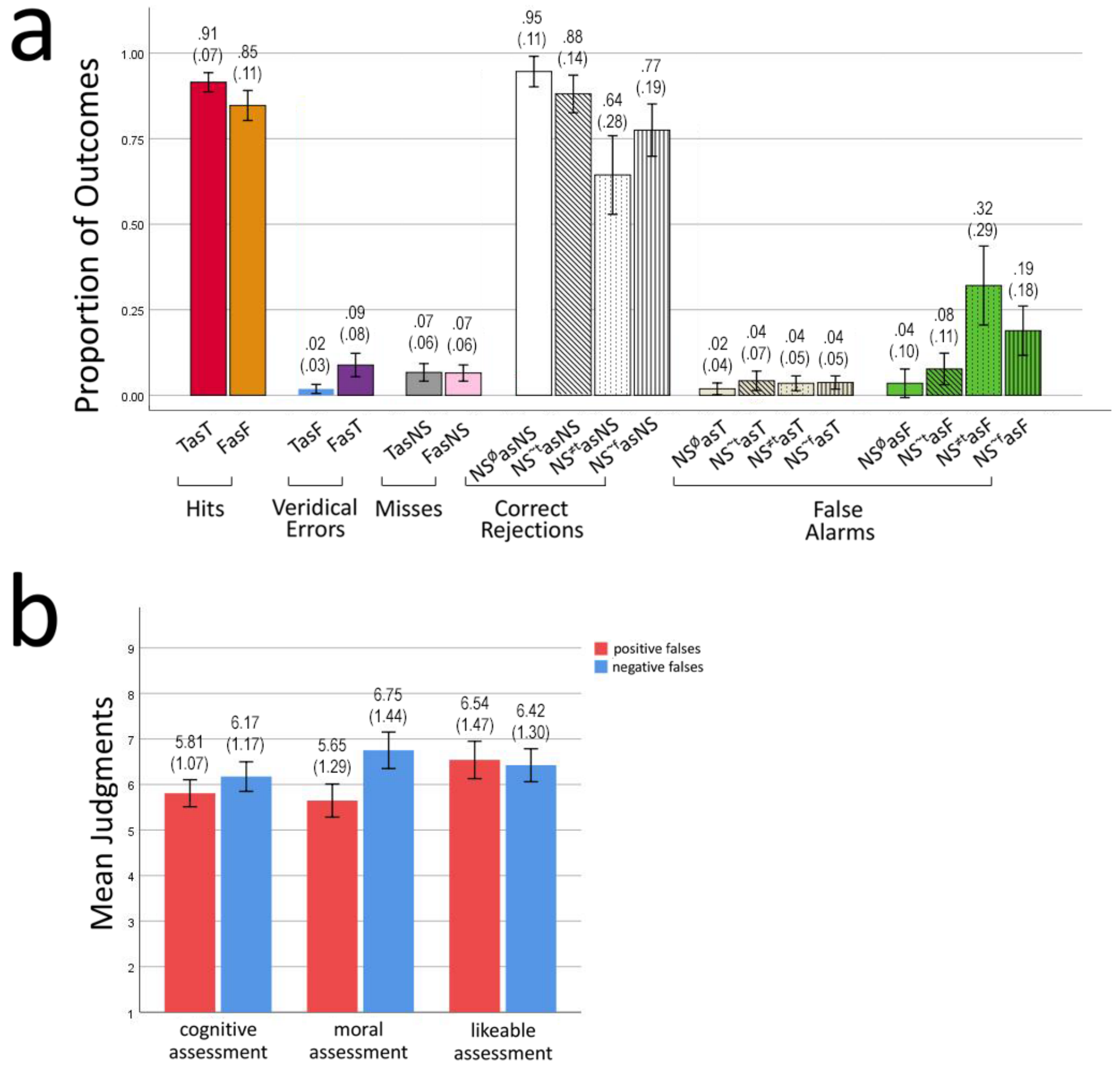
Across both graphs means and standard deviations are given above each bar. Error bars indicate $95 \%$ confidence intervals. a) True/false recognition memory test response rates across all Study 1 participants. The $\mathrm{Y}$ axis is the proportion of responses across all 4 DJT stories. TasT represents true statements correctly identified as true; FasF represents false statements correctly identified as false; TasF represents true statements incorrectly identified as false; FasT represents false statements incorrectly identified as true; TasNS represents true statements incorrectly identified as never seen; FasNS represents false statements incorrectly identified as never seen; $\mathrm{NS}^{\varnothing}$ asNS represents not related foil statements correctly identified as never seen; $\mathrm{NS}^{\sim} \mathrm{t}$ asNS represents similar to true foil statements correctly identified as never seen; $\mathrm{NS}^{\neq \mathrm{t}}$ asNS represents mutually exclusive to true foil statements correctly identified as never seen; $\mathrm{NS}^{\sim \mathrm{f}}$ asNS represents similar to false foil statements correctly identified as never seen; $\mathrm{NS}^{\emptyset_{\text {asT }}}$ represents not related foil statements incorrectly identified as true; $\mathrm{NS}^{\frown \uparrow}$ asT represents similar to true foil statements incorrectly identified as true; $\mathrm{NS}^{\neq t}$ asT represents mutually exclusive to true foil statements incorrectly identified as true; $\mathrm{NS}^{\sim \mathrm{f}}$ asT represents similar to false foil statements incorrectly identified as true; $\mathrm{NS}^{\varnothing}$ asF represents not related foil statements incorrectly identified as false; $\mathrm{NS}^{\sim}$ asF represents similar to true foil statements incorrectly identified as false; $\mathrm{NS}^{\neq t}$ asF represents mutually exclusive to true foil statements incorrectly identified as false; and $\mathrm{NS}^{\sim f}$ asF represents similar to false foil statements incorrectly identified as false. Of note, FasT > TasF and $\mathrm{NS}^{\ddagger t}$ asF $>$

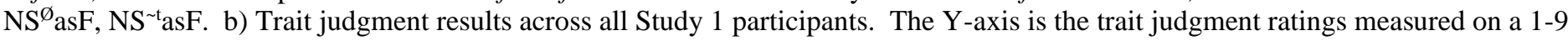
Likert scale. The cognitive assessment included ratings of intelligence and cleverness to the protagonists Mike (positive explicitlylabeled false statements) and Brad (negative explicitly-labeled false statements); the moral assessment included ratings of honesty and sincerity to the protagonists John (negative explicitly-labeled false statements) and Tom (positive explicitly-labeled false statements); and the likeable assessment include ratings of likability to Mike, John, Brad, and Tom. Red bars indicate that the story contained explicitly-labeled false information that (if believed) would increase the trait judgment rating (positive falses), and blue bars indicate that the story contained explicitly-labeled false information that (if believed) would decrease the trait judgment rating (negative falses). Of note, false cues moderated the trait judgment ratings (see Figure $7 \mathrm{~b}$ for a comparison). 
Figure 4

Study 2 DJT results

a

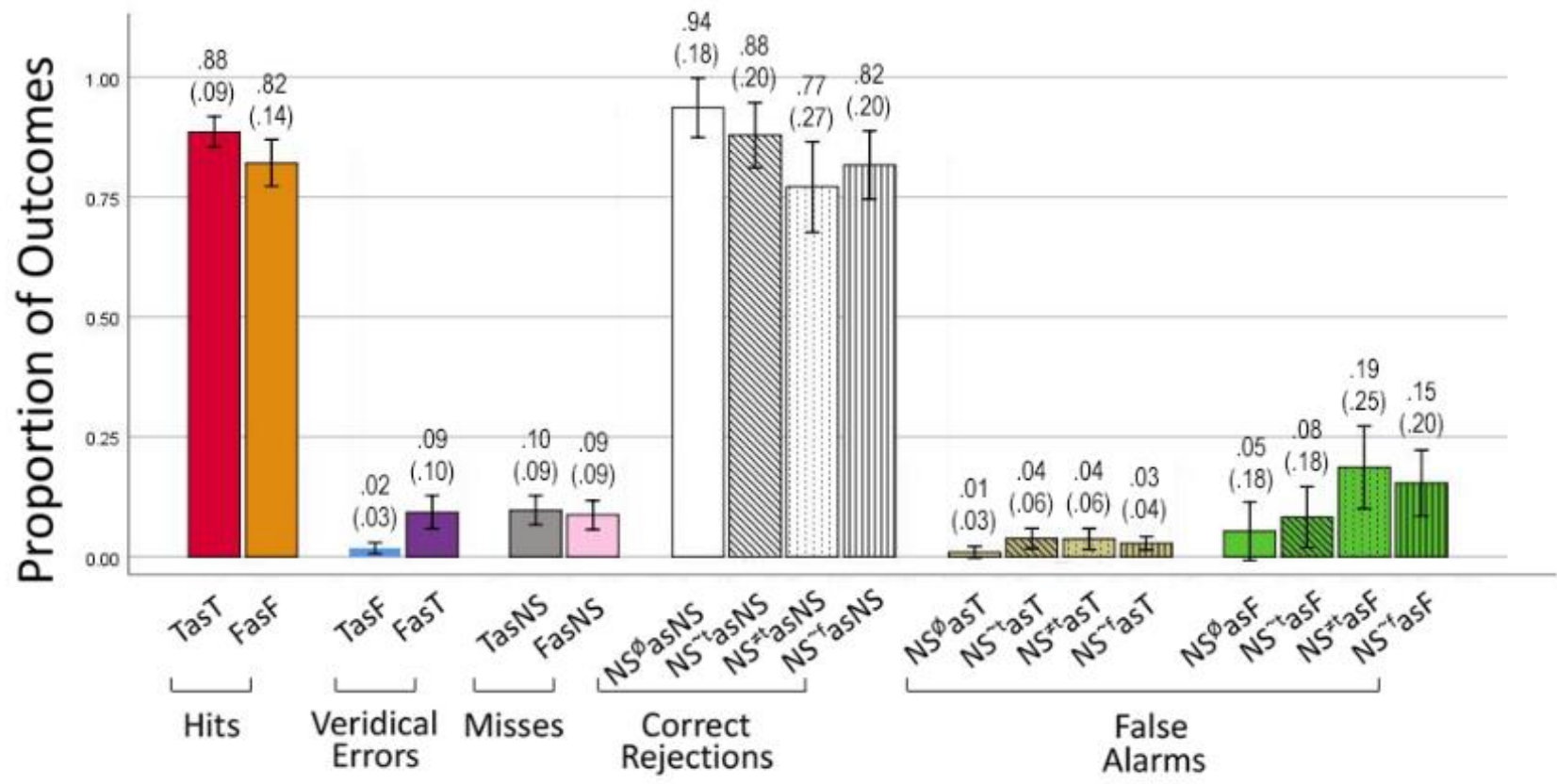

b

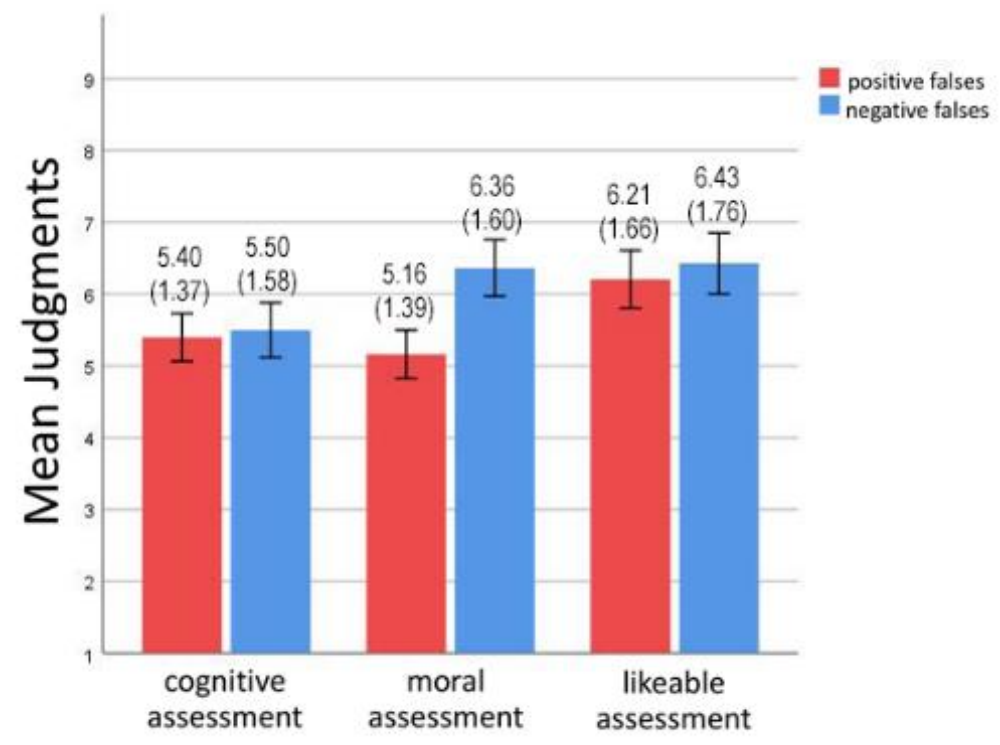

Across both graphs means and standard deviations are given above each bar. Error bars indicate $95 \%$ confidence intervals. a) True/false recognition memory test response rates across all Study 2 participants. The $\mathrm{Y}$ axis is the proportion of responses across all 4 DJT stories. See text or Figure 3a legend for individual bar labeling descriptions. Of note, FasT $>$ TasF and NS ${ }^{\neq t}$ asF $>N^{\varnothing}{ }^{\varnothing}$ asF, $\mathrm{NS}^{-\mathrm{t}}$ asF. b) Trait judgment results across all Study 2 participants. The $\mathrm{Y}$-axis is the trait judgment ratings measured on a 1-9 Likert scale. See text or Figure $3 b$ legend for individual bar labeling descriptions. Red bars indicate that the story contained explicitlylabeled false information that (if believed) would increase the trait judgment rating (positive falses), and blue bars indicate that the story contained explicitly-labeled false information that (if believed) would decrease the trait judgment rating (negative falses). Of note, false cues moderated the trait judgment ratings (see Figure $7 \mathrm{~b}$ for a comparison). 
Figure 5

Structure and parameters of the HLT MPT models

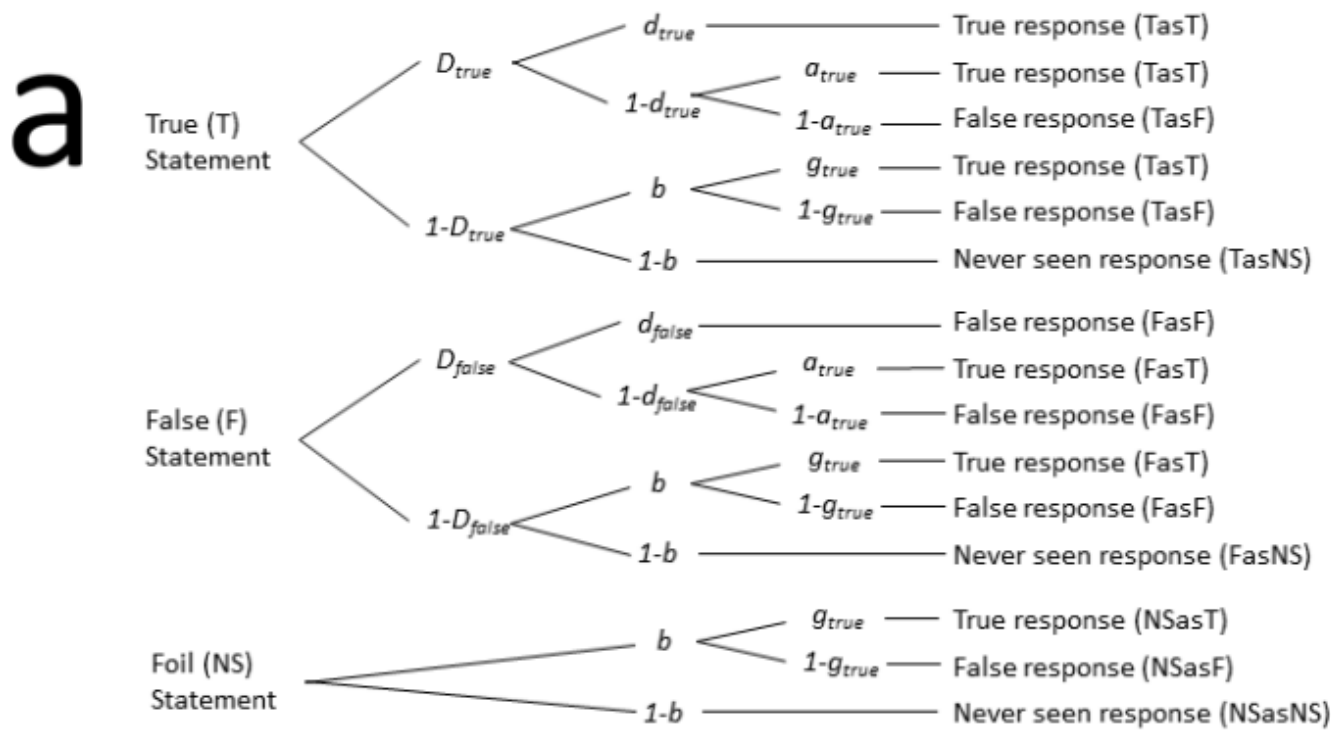

b

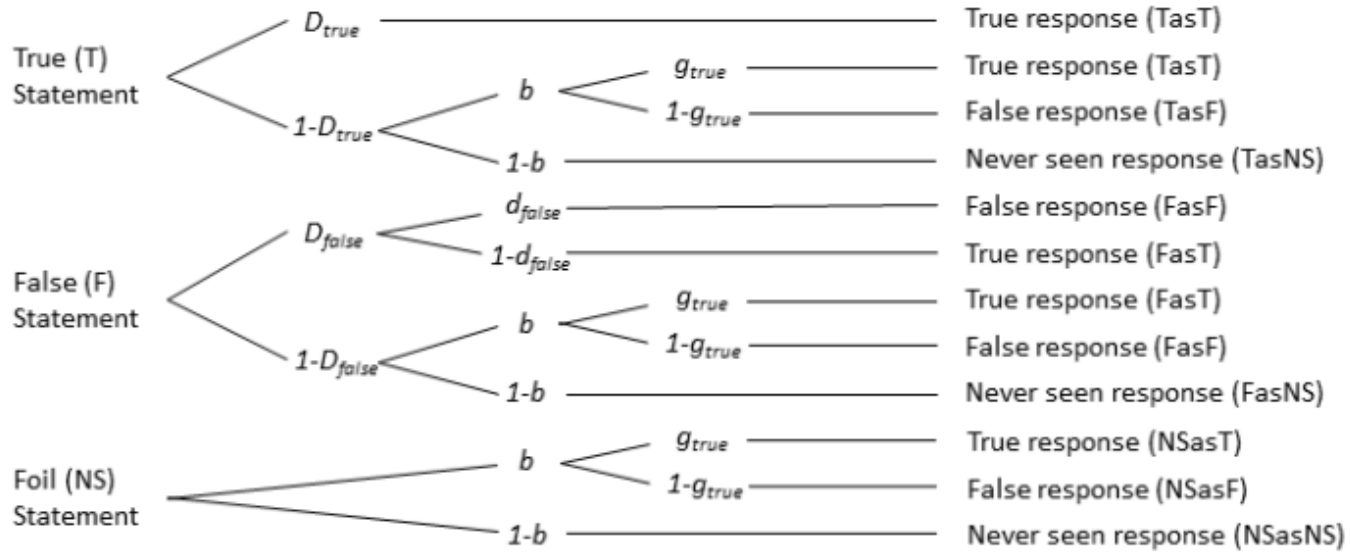

Each model consists of separate processing trees for true, false, and foil statements. Tree branches represent a possible sequence of cognitive processes resulting in a true, false, or never seen response. Model parameters reflect transition probabilities from left to right. a) HLT Cartesian MPT model. b) HLT belief-default MPT model. See text or Figure 2 legend for parameter descriptions. Of note, the HLT belief-default MPT model does not contain a $d_{\text {true }}$ parameter. HLT data from Study 3 fit the Cartesian MPT model well and fit the belief-default MPT model poorly. 
Figure 6

Study 3 DJT and HLT results
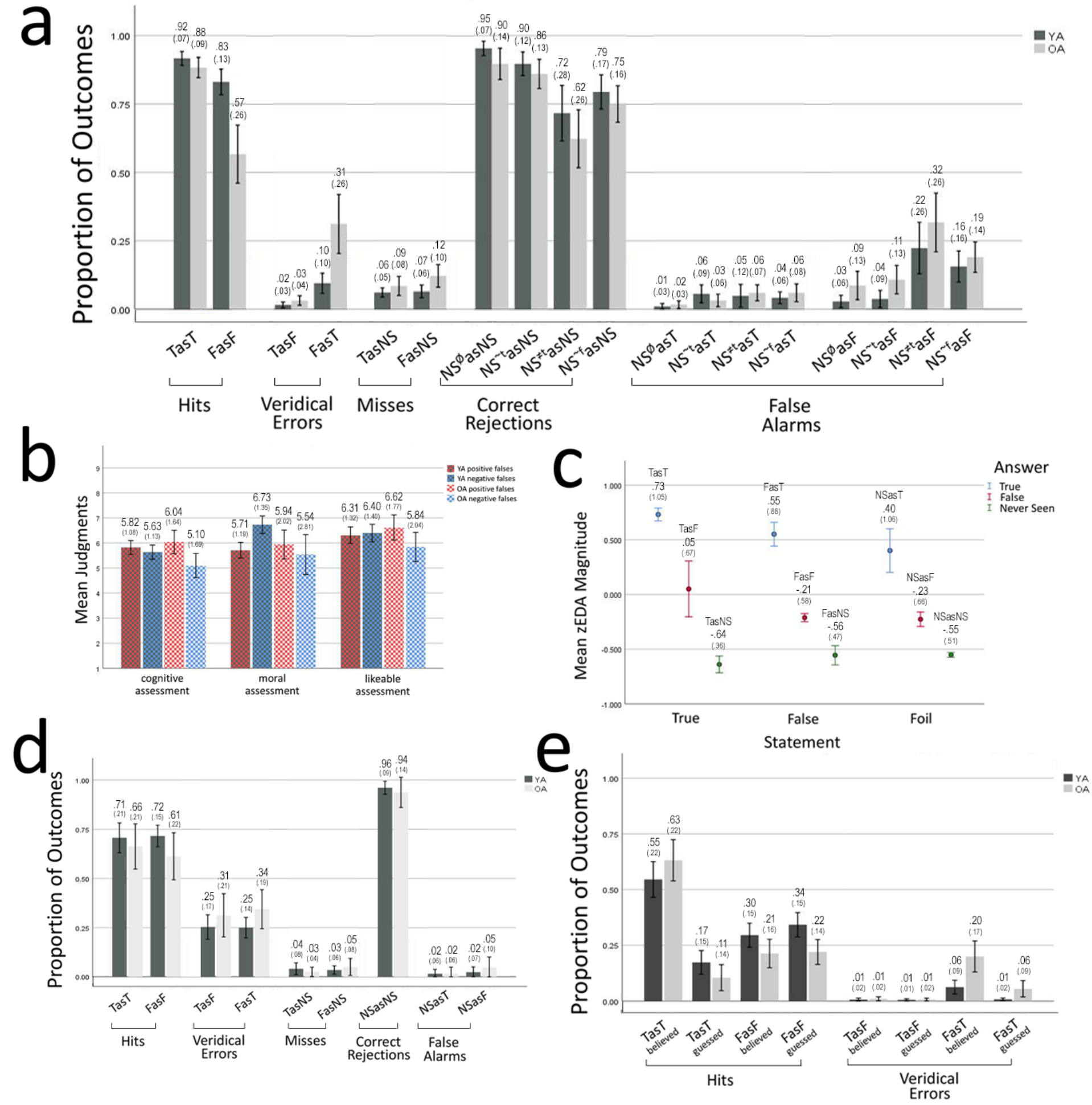
Across all graphs means and standard deviations are given. Error bars indicate 95\% confidence intervals. YA, younger adults; OA, older adults. a) DJT true/false recognition memory test response rates across Study 3 participants. The Y axis is the proportion of responses across all 4 DJT stories. See text or Figure 3 a legend for individual bar labeling descriptions. Of note, in both younger and older adults, FasT $>$ TasF and $\mathrm{NS}^{\ddagger t} \mathrm{asF}>\mathrm{NS}{ }^{\varnothing} \mathrm{asF}, \mathrm{NS}^{\sim \mathrm{t}} \mathrm{asF}$; and older adults commit significantly more FasT errors than younger adults. b) DJT trait judgment results across Study 3 participants. The Y-axis is the trait judgment ratings measured on a 1-9 Likert scale. See text or Figure $3 b$ legend for individual bar labeling descriptions. Red bars indicate that the story contained explicitlylabeled false information that (if believed) would increase the trait judgment rating (positive falses), and blue bars indicate that the story contained explicitly-labeled false information that (if believed) would decrease the trait judgment rating (negative falses). Of note, false cues failed to moderated the trait judgment ratings in older adults relative to false cue moderation in younger adults (see Figure $7 b$ for a comparison). c) DJT zEDA magnitude results across all Study 3 participants. Foil statement results were collapsed together for ease of presentation (see Supplementary Materials for a DJT old and foil non-collapsed statement zEDA analysis). Y-axis represents zEDA magnitudes; $\mathrm{X}$-axis represents the objective classification of items presented during the DJT true/false recognition memory test; and color represents the answer provided by participants: blue is true responding, red is false responding, and green is never seen responding. Of note, there was a main effect of answering such that true responding corresponded with the highest zEDA magnitudes, false responding corresponded with middle zEDA magnitudes, and never seen responding corresponded with the lowest zEDA magnitudes. d) HLT true/false recognition memory test response rates across Study 3 participants. The Y axis is the proportion of responses across both HLT recognition memory test sessions. See text or Figure 3a legend for individual bar labeling descriptions. Of note, in both younger and older adults, FasT $\sim$ TasF. e) Post-hoc DJT hit and veridical error true/false recognition memory test zEDA "believed" versus "guessed" analysis. The Y axis is the proportion of responses across all 4 DJT stories. See text or Figure 3a legend for individual bar labeling descriptions. A true response was categorized as "believed" if the zEDA was greater than .25 and a false response was categorized as "believed" if the zEDA was between -.39 and .25. True and false responses were categorized as "guessed" if the zEDA was below -.39. Of note, in both younger and older adults "believed" FasT $>$ "believed" TasF, "guessed" FasT and older adults had greater "believed" FasT relative to younger adults. 
Figure 7

Study 4 SM-DJT results
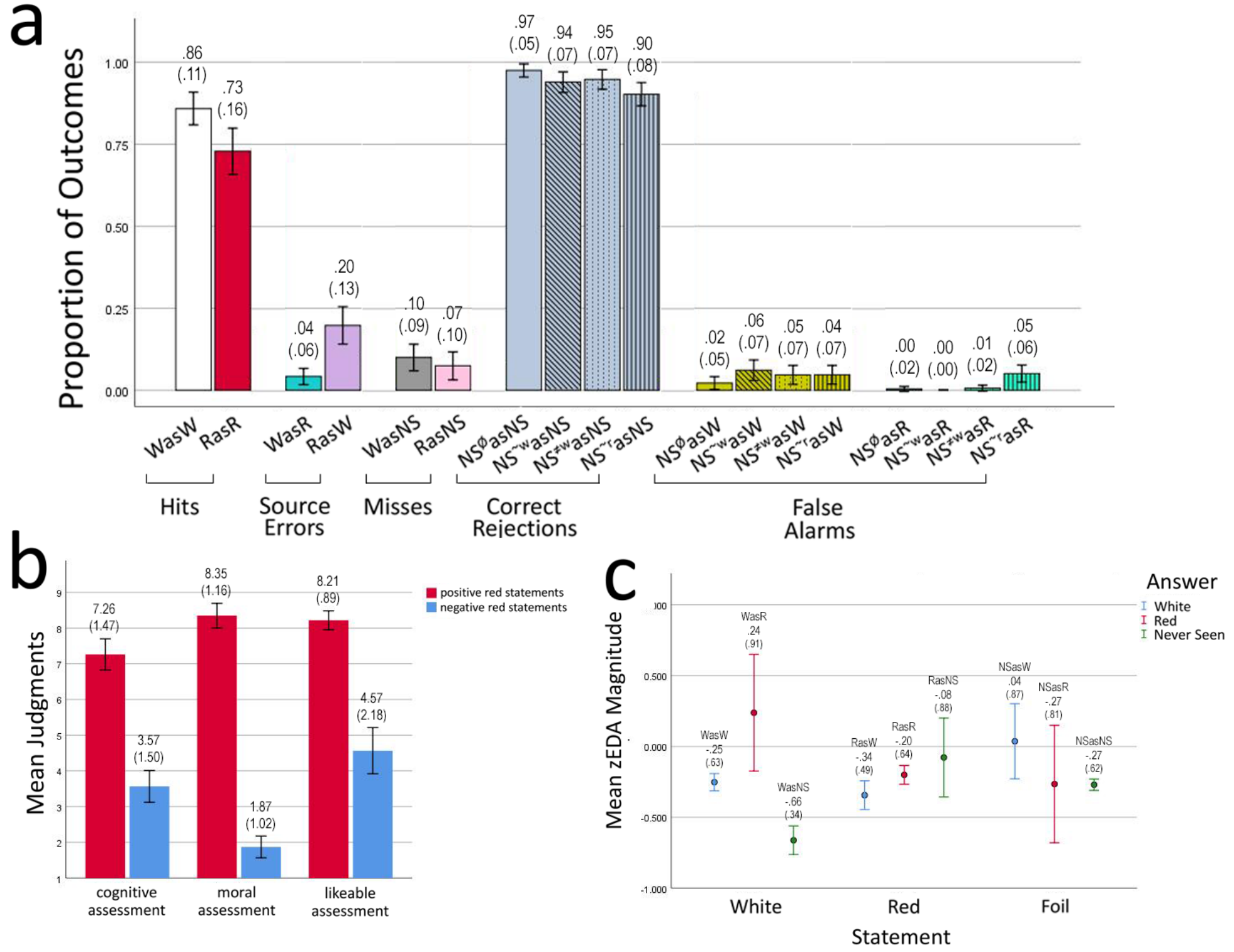
Across all graphs means and standard deviations are given. Error bars indicate 95\% confidence intervals. a) SM-DJT white/red recognition memory test response rates across all Study 4 participants. The Y axis is the proportion of responses across all 4 SM-DJT stories. WasW represents white statements correctly identified as white; RasR represents red statements correctly identified as red; WasR represents white statements incorrectly identified as red; RasW represents red statements incorrectly identified as white; WasNS represents white statements incorrectly identified as never seen; RasNS represents red statements incorrectly identified as never seen; $\mathrm{NS}^{\varnothing}$ asNS represents not related foil statements correctly identified as never seen; $\mathrm{NS}^{\sim \mathrm{w}}$ asNS represents similar to white foil statements correctly identified as never seen; $\mathrm{NS}^{\neq \mathrm{w}}$ asNS represents mutually exclusive to white foil statements correctly identified as never seen; $\mathrm{NS}^{\sim}$ asNS represents similar to red foil statements correctly identified as never seen; $\mathrm{NS}^{\varnothing}$ asW represents not related foil statements incorrectly identified as white; $\mathrm{NS}^{\sim \mathrm{w}}$ asW represents similar to white foil statements incorrectly identified as white; $\mathrm{NS}^{\neq \mathrm{w}}$ asW represents mutually exclusive to white foil statements incorrectly identified as white; $\mathrm{NS}^{\sim \mathrm{r}}$ asW represents similar to red foil statements incorrectly identified as white; $\mathrm{NS}^{\varnothing}$ asR represents not related foil statements incorrectly identified as red; $\mathrm{NS}^{\sim \mathrm{w}}$ asR represents similar to white foil statements incorrectly identified as red; $\mathrm{NS}^{\neq \mathrm{w}}$ asR represents mutually exclusive to white foil statements incorrectly identified as red; and $\mathrm{NS}^{\sim \sim}$ asR represents similar to red foil statements incorrectly identified as red. Of note, $\mathrm{NS}^{\neq \mathrm{w}}$ asR $\mathrm{NS}^{\varnothing}$ asR, NS ${ }^{\sim}$ asR. b) SM-DJT trait judgment results across Study 4 participants. The Y-axis is the trait judgment ratings measured on a 1-9 Likert scale. See text or Figure 3b legend for individual bar labeling descriptions. Red bars indicate that the story contained information that was in red text that (when believed) would increase the trait judgment rating (positive reds), and blue bars indicate that the story contained information that was in red text that (when believed) would decrease the trait judgment rating (negative reds). Of note, information presented in red during the SM-DJT deflected trait judgments in the designed direction (see Figure 3b, 4b, 6b for comparisons). c) SM-DJT zEDA magnitude results across all Study 4 participants. Foil statement results were collapsed together for ease of presentation. Y-axis represents zEDA magnitudes; X-axis represents the objective classification of items presented during the SM-DJT white/red recognition memory test; and color represents the answer provided by participants: blue is white responding, red is red responding, and green is never seen responding. Of note, there was no main effect of answering nor of correct responses (WasW $\sim$ RasR NSasNS; see Figure 6c for a comparison). 